\title{
MICROSTRUCTURAL MODELING DURING MULTI-PASS ROLLING OF A NICKEL-BASE SUPERALLOY
}

by

Kannan Subramanian

A dissertation submitted to the faculty of The University of North Carolina at Charlotte in partial fulfillment of the requirements for the degree of Doctor of Philosophy in Mechanical Engineering

Charlotte

2009

Approved by:

Dr. Harish P. Cherukuri

Dr. Ramesh S. Minisandram

Dr. Kingshuk Bose

Dr. Wei Cai

Dr. Animikh Biswas 
(C) 2009

Kannan Subramanian

ALL RIGHTS RESERVED 
KANNAN SUBRAMANIAN: Microstructural Modeling During Multi-Pass Rolling of a Nickel-Base Superalloy. (Under the direction of DR. HARISH P. CHERUKURI)

Microstructure present at the end of rolling and cooling operations controls the product properties. Therefore, control of grain size is an important characteristic in any hot-working. The narrow temperature range for hot working of Alloy 718 makes the grain size control more difficult. In the current work, a systematic numerical approach to predict the microstructure of Alloy 718 during multi-pass rolling is developed. This approach takes into account the severe deformation that takes place during each pass and also the possible reheating between passes. In order to predict the grain size at the end of rolling process, microstructural processes such as dynamic recrystallization (DRX), metadynamic recrystallization (MDRX), and static grain growth need to be captured at every deformation step for superalloys. Empirical relationships between the average grain size from various microstructural processes and the macroscopic variables such as temperature $(T)$, effective strain $(\bar{\varepsilon})$ and strain rate $(\dot{\bar{\varepsilon}})$ form the basis for the current work. The empirical relationships considered in this work are based on Avrami equations and utilize data taken from various forging analyses. The macroscopic variables are calculated using the Finite Element Method (FEM) by modeling the rolling process as a creeping flow problem. FEM incorporates a mesh re-zoning algorithm that enables the analysis to continue for several passes. A two-dimensional transient thermal analysis is carried out between passes that can capture the MDRX and/or static grain growth during the microstructural evolution. The microstructure prediction algorithm continuously updates two families of grains, namely, the recrystallized family and strained family at the start of deformation in any given pass. In addition, the algorithm calculates various subgroups within these two families at every deformation step within a pass. As the material undergoes deformation between the rolls, recrystallization equations are invoked depending on critical strain and strain rate conditions that are characteristics of Alloy 718. This approach predicts the microstructural evolution based on recrystallization kinetics 

and static grain growth only. Precipitation of phases such as $\gamma^{\prime}, \gamma^{\prime \prime}$ and $\delta$ are not considered. Modeling this complex precipitation is difficult and requires a more detailed understanding than is presently available. Nevetheless, comparisons of the grain sizes from the proposed numerical models with experimental results for 16-stand rolling process show very good agreement. 


\section{ACKNOWLEDGEMENTS}

I express my sincere gratitude to my thesis advisor, Dr. Harish P. Cherukuri for his mentoring and support throughout my graduate program at UNC-Charlotte. I thank Dr. Ramesh S. Minisandram for providing us the wonderful opportunity to work on this project and guidance through this work. The financial support from ATI Allvac, Monroe is greatly appreciated. Dr. J. P. Thomas has been very helpful in providing extensive information about the behavior of Alloy 718 material and helped in fine tuning the technical aspects of this dissertation.

I thank Dr. Kingshuk Bose for his valuable suggestions during the development of mesh re-zoning algorithm. I thank my other committee members Dr. Wei Cai and Dr. Animikh Biswas for their valuable time. It is very difficult for me to provide the long list of names of the friends who have helped me on various occassions during my graduate study. It is a great pleasure knowing them all and their help is immensely acknowledged. I am grateful to my sisters and brother for their encouragement and strong support. I dedicate this work to my parents whose hard work and perseverance has been instrumental in all my academic achievements. 


\section{TABLE OF CONTENTS}

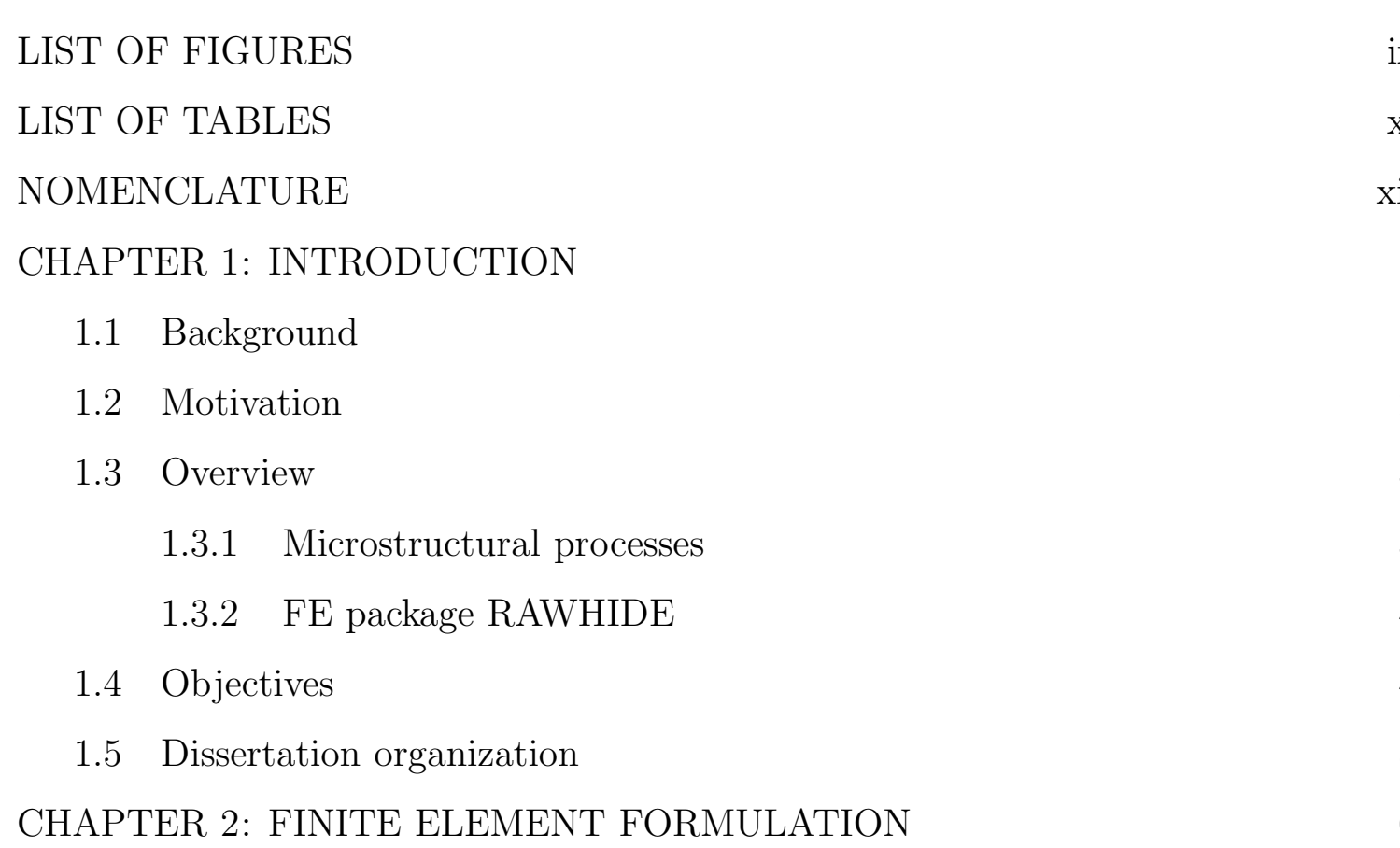

2.1 RAWHIDE Introduction 6

$\begin{array}{lll}2.2 & \text { Variational flow formulation } & 7\end{array}$

$\begin{array}{lll}2.2 .1 \quad \text { Field equations } & 11\end{array}$

2.3 FE flow formulation 14

$\begin{array}{ll}\text { 2.3.1 Matrix form } & 15\end{array}$

$\begin{array}{ll}\text { 2.3.2 Galerkin's formulation } & 15\end{array}$

2.4 Heat transfer formulation 18

2.5 Mesh generation 20

2.6 RAWHIDE Capabilities 20

CHAPTER 3: MESH RE-ZONING IN MULTI-STAND ROLLING 22

$\begin{array}{lll}3.1 & \text { Introduction } & 22\end{array}$

3.2 Mesh distortion 22

3.3 Outline of the mesh re-zoning approach 24

\begin{tabular}{ll}
3.4 Cubic splines & 25 \\
\hline \hline
\end{tabular}

3.5 Re-meshing 26

$\begin{array}{lll}3.5 .1 & \text { Influence on area } & 29\end{array}$

3.6 Interpolation 30 
3.7 Results from mesh re-zoning 35

3.8 Interpass temperature history 36

$\begin{array}{ll}3.9 \text { Conclusion } & 37\end{array}$

CHAPTER 4: MICROSTRUCTURE THEORY AND RELATED WORK 40

4.1 Introduction 40

$\begin{array}{lll}4.1 .1 & \text { Recovery } & 40\end{array}$

4.1.2 Recrystallization 41

4.1.3 Parameters 41

4.2 Microstructural evolution 43

$\begin{array}{lll}4.2 .1 & \text { DRX } & 44\end{array}$

$\begin{array}{lll}4.2 .2 \text { MDRX } & 44\end{array}$

\begin{tabular}{ll}
$4.2 .3 \quad \mathrm{SRX}$ & 44 \\
\hline
\end{tabular}

$\begin{array}{lll}\text { 4.2.4 SGG } & 45\end{array}$

4.3 Empirical modeling $\quad 45$

4.4 Related work 45

$\begin{array}{lll}4.4 .1 & \text { Constitutive laws } & 45\end{array}$

$\begin{array}{lll}\text { 4.4.2 Computations } & 47\end{array}$

$\begin{array}{lll}4.4 .3 & \text { Formulations } & 48\end{array}$

$\begin{array}{lll}4.4 .4 & \text { Precipitates } & 49\end{array}$

$\begin{array}{ll}\text { 4.4.5 Atomistic approaches } & 50\end{array}$

CHAPTER 5: MICROSTRUCTURE ALGORITHM 52

5.1 Introduction $\quad 52$

5.2 Formulation $\quad 53$

5.3 Implementation $\quad 57$

$\begin{array}{ll}\text { 5.3.1 Dynamic recrystallization } & 57\end{array}$

5.3.2 Metadynamic recrystallization $\quad 62$

$\begin{array}{lll}\text { 5.3.3 Static grain growth } & 64\end{array}$

CHAPTER 6: RESULTS AND DISCUSSIONS 67

$\begin{array}{lll}6.1 & \text { Introduction } & 67\end{array}$

$\begin{array}{lll}6.2 \text { Cooling } & 68\end{array}$ 
6.3 4-Stand analysis with air cooling at the end of $4^{\text {th }}$ stand $\quad 69$

$\begin{array}{lll}\text { 6.3.1 Initial observations } & 71\end{array}$

\begin{tabular}{ll}
6.3 .2 & Streamline results \\
\hline
\end{tabular}

$\begin{array}{lll}\text { 6.3.3 Final observations } & 74\end{array}$

6.4 16-Stand analysis with air cool and water quenching after $16^{\text {th }}$ stand $\quad 80$ $\begin{array}{lll}6.4 .1 & \text { Streamline results } & 81\end{array}$

$\begin{array}{lll}6.4 .2 & \text { Final observations } & 84\end{array}$

6.5 Discussions 84

6.6 Finer mesh results $\quad 93$

6.7 Conclusion 94

CHAPTER 7: CONCLUSIONS \& FUTURE WORK 96

7.1 Summary of the present work $\quad 96$

$\begin{array}{ll}7.2 \text { Conclusions } & 96\end{array}$

$\begin{array}{lll}7.3 & \text { Future Work } & 97\end{array}$

$\begin{array}{ll}\text { BIBLIOGRAPHY] } & 98\end{array}$ 


\section{LIST OF FIGURES}

FIGURE 2.1: Thermo-mechanical coupling. $\quad 7$

FIGURE 2.2: 3D and 2D analysis control volumes. 19

FIGURE 2.3: Boundary conditions used for the 3D analysis. 19

FIGURE 2.4: Creation of FE mesh used in the multi-pass rolling simulation. 20

FIGURE 2.5: Steady state results from FEM. 21

FIGURE 3.1: Distorted mesh during multi-stand rolling of Alloy 718. 23

FIGURE 3.2: Boundary extraction from the FE mesh. 24

FIGURE 3.3: Various spline fits for the mesh boundary. $\quad 25$

FIGURE 3.4: Creation of new FE mesh inner layers. 27

FIGURE 3.5: Creation of new FE mesh outer layer. $\quad 27$

FIGURE 3.6: Redefined mesh during multi-stand rolling of alloy 718.

FIGURE 3.7: Re-meshed \& interpolated contour comparison. 30

FIGURE 3.8: Strain contours.

FIGURE 3.9: Comparison of predicted shapes. 36

FIGURE 3.10: Temperature history output. 38

FIGURE 3.11: Reduction in area during continuous rolling. 39

FIGURE 4.1: Micrograph showing 'necklace' structure. $\quad 42$

FIGURE 4.2: DRX based on Strain. 43

FIGURE 5.1: Typical streamlines from the 3D FE analysis. 53

FIGURE 5.2: Microstructure families (based on Reference [61]). 54

FIGURE 5.3: Microstructure evolution of different families. $\quad 56$

FIGURE 5.4: Nodes on a streamline. $\quad 57$

FIGURE 5.5: DRX based on strain. 58

FIGURE 5.6: MDRX based on time. 63

FIGURE 5.7: Microstructure algorithm. 66

FIGURE 6.1: Mesh considered and the schematic control volume in a pass. 68

FIGURE 6.2: Temperature history on various streamlines (4-stand analysis). 69

FIGURE 6.3: Strain history on various streamlines (4-stand analysis). 70 
FIGURE 6.4: Recrystallization fractions after $1^{\text {st }}$ stand and insterstands. $\quad \begin{array}{r}\mathrm{x} \\ 70\end{array}$

FIGURE 6.5: Recrystallization fractions at the end of $2^{\text {nd }}$ stand. $\quad 71$

FIGURE 6.6: Deformation variables from the FE analysis after $2^{\text {nd }}$ stand. $\quad 72$

FIGURE 6.7: Recrystallization fractions at the end of $2^{\text {nd }}$ interstand. $\quad 73$

FIGURE 6.8: Central streamline (•) results for a 4-stand analysis. $\quad 75$

FIGURE 6.9: First midradius streamline $(\bullet)$ results for a 4-stand analysis. 76

FIGURE 6.10: Second midradius streamline (A) results for a 4-stand analysis. $\quad 77$

FIGURE 6.11: Subsurface streamline $(\boldsymbol{\nabla})$ results for a 4-stand analysis. $\quad 78$

FIGURE 6.12: Surface streamline (ם) results for a 4-stand analysis. 79

FIGURE 6.13: Recrystallization fractions during and after $4^{\text {th }}$ stand. $\quad 80$

FIGURE 6.14: REX family grain size after air cooling at the end of $4^{\text {th }}$ stand. $\quad 81$

FIGURE 6.15: Micrograph results at the end of $4^{\text {th }}$ stand. $\quad 82$

FIGURE 6.16: Temperature history on various streamlines (16-stand analysis). 83

FIGURE 6.17: Strain history on various streamlines (16-stand analysis). $\quad 85$

FIGURE 6.18: Central streamline (๑) results for a 16-stand analysis. 86

FIGURE 6.19: First midradius streamline $(\bullet)$ results for a 16-stand analysis. 87

FIGURE 6.20: Second midradius streamline (A) results for a 16-stand analysis. 88

FIGURE 6.21: Subsurface streamline $(\boldsymbol{\nabla})$ results for a 16-stand analysis. $\quad 89$

FIGURE 6.22: Surface streamline (ם) results for a 16-stand analysis. 90

FIGURE 6.23: Grain sizes $(\mu \mathrm{m})$ at the end of cooling after $16^{\text {th }}$ stand. $\quad 91$

FIGURE 6.24: Micrograph results at the end of $16^{\text {th }}$ stand. $\quad 92$

FIGURE 6.25: Finer mesh considered for microstructure comparison. 92

FIGURE 6.26: Grain sizes after $16^{\text {th }}$ stand for finer mesh. 93

FIGURE 6.27: Temperature comparison for different meshes. $\quad 94$ 


\section{LIST OF TABLES}

TABLE 3.1: Area increase due to remeshing. 30

TABLE 3.2: Predicted Vs. Measured. 


\section{NOMENCLATURE}

$C_{p} \quad$ Specific heat

$D R X$ Dynamic Recrystallization

$D_{\text {rex }} \quad$ Grain size of recrystallized grains due to averaging

$D_{s t} \quad$ Grain size of the strained grains due to averaging

$F_{\text {rex }} \quad$ Fraction of recrystallized grains due to averaging

$F_{s t} \quad$ Fraction of strained grains due to averaging

$J \quad$ Potential Energy Functional

$J M A K$ Johnson-Mehl-Avrami-Kolmogorov

MDRX Metadynamic Recrystallization

$N_{i} \quad$ Shape functions evaluated at node $i$

$Q \quad$ Activation energy of a process

$Q_{\text {dynd }}$ Activation energy for DRX in evaluating the steady state grain size

$Q_{d y n h}$ Activation energy for DRX in evaluating $\bar{\varepsilon}_{0} .5$

$Q_{g g r}$ Activation energy for grain growth

$Q_{m d y n d}$ Activation energy for MDRX in evaluating the steady state grain size

$Q_{m d y n h}$ Activation energy for MDRX in evaluating $t_{0} .5$

$R \quad$ Universal gas constant

Re Reynold's Number

$S G G$ Static Grain Growth

$S R X$ Static Recrystallization 
T Temperature

$W \quad$ Work done during deformation

$X_{i} \quad$ Body force per unit mass

Z Zener-Hollomon Parameter

$\Gamma \quad$ Boundary

$\Gamma_{q} \quad$ Flux defined boundary

$\Gamma_{t} \quad$ Traction specified boundary

$\Gamma_{u} \quad$ Velocity specified boundary

$\Gamma_{\theta} \quad$ Temperature defined boundary

$\Omega \quad$ Domain

$\bar{T}_{i} \quad$ Component of traction vector

$\bar{T}_{x, y, z}$ Traction components

$\bar{\theta} \quad$ Defined temperature

$\bar{\varepsilon} \quad$ Effective strain

$\bar{\varepsilon}_{0.5} \quad$ Strain at which the grains are $50 \%$ recrystallized

$\bar{\varepsilon}_{c} \quad$ Critical strain

$\bar{\varepsilon}_{p} \quad$ Peak strain

$\bar{\varepsilon}_{\text {rex }}$ Instantaneous effective strain for the recrystallized grain family

$\bar{\varepsilon}_{s t} \quad$ Instantaneous effective strain for the strained grain family

$\bar{\varepsilon}_{v} \quad$ Virtual strain

$\bar{q} \quad$ Defined flux 
$\beta \quad$ Taylor-Quinney coefficient

$\boldsymbol{B}^{u} \quad$ Derivative of the shape functions matrix for nodal velocity

$\boldsymbol{B}^{\theta} \quad$ Derivative of the shape functions matrix for nodal temperature

$D \quad$ Viscosity matrix

I Second order identity tensor

$\boldsymbol{N}^{\prime}$ Derivative of the shape functions matrix for nodal velocity

$\boldsymbol{N}^{p} \quad$ Shape function matrix for pressure

$\boldsymbol{N}^{u} \quad$ Shape function matrix for velocity

$\boldsymbol{N}^{\theta} \quad$ Shape function matrix for nodal temperature

$\overline{\boldsymbol{T}} \quad$ Traction vector

$\dot{\varepsilon} \quad$ Vectorial form of strain rate tensor

$\hat{\boldsymbol{p}} \quad$ Nodal pressures

$\hat{\boldsymbol{u}} \quad$ Nodal velocity vector

$\nabla \quad$ Gradient operator

$\boldsymbol{\sigma}^{\prime} \quad$ Vectorial form of deviatoric stress tensor

$\boldsymbol{f} \quad$ Force vector

$\boldsymbol{k}_{d} \quad$ Stiffness matrix for velocity

$\boldsymbol{m} \quad$ Vectorial form of identity matrix

$\boldsymbol{q} \quad$ Stiffness matrix for pressure

$\boldsymbol{u} \quad$ Velocity vector

$\delta a \quad$ Small increment or variation in any quantity $a$ 
$\delta_{i j} \quad$ Component of the identity tensor

$\dot{Q} \quad$ Heat generation rate

$\dot{\bar{\varepsilon}} \quad$ Effective strain rate

$\dot{\varepsilon}_{i j} \quad$ Component of strain rate tensor

$\dot{\varepsilon}_{i j}{ }^{\prime} \quad$ Component of deviatoric strain rate tensor

$\epsilon \quad$ Emissivity

$\frac{\partial}{\partial a} \quad$ Partial derivative with respect to the quantity $a$

$\gamma \quad$ Penalty parameter

$\kappa \quad$ Boltzmann's Constant

$\lambda \quad$ Lagrange multiplier

H Global stiffness matrix for heat transfer analysis

$\mathbf{K}_{D} \quad$ Global stiffness matrix for velocity

Q Global stiffness matrix for pressure

A Assembly operator

$\theta \quad$ Temperature variable column matrix

b Body force vector

f $\quad$ Force vector

q Force vector for heat transfer analysis

u Global veocity variables

$\mathbf{u}_{\text {roller }}$ Veocity of the roller

$\mu \quad$ Viscosity 
$\nu \quad$ Fraction to find the retained strain

$\bar{T} \quad$ Average temperature used in microstructure calculations

$\rho \quad$ Density

$\sigma_{i j}^{\prime} \quad$ Component of the deviatoric stress tensor

$\sigma_{y} \quad$ Yield Stress

$\sigma_{i j} \quad$ Component of the stress tensor

$\tau \quad$ Strain rate multiplier

$\theta \quad$ Temperature variable

$\theta_{e} \quad$ Temperature of the fluid

$\theta_{s} \quad$ Temperature at the surface

$\xi, \eta \quad$ Local coordinates in FE formulation

n Iteration number

$a^{T} \quad$ Transpose of the quantity $a$

$a^{e} \quad$ Superscript $e$ represents the quantity $a$ at the element level

$a_{, i} \quad$ Derivative of $a$ with respect to $x_{i}$

$a_{d y n} \quad$ Temperature specific DRX parameter to evaluate $X$ and $\bar{\varepsilon}_{0.5}$

$a_{i i} \quad$ Summation implied over the index $i$ implied on any quantity $a$

$a_{m d y n}$ MDRX parameter to evaluate $X$ and $t_{0.5}$

$b_{m d y n}$ MDRX parameter to evaluate $X$ and $t_{0.5}$

$c_{d y n}$ Temperature specific DRX parameter to evaluate $\bar{\varepsilon}_{p}$

$c_{d y n} \quad$ Temperature specific DRX parameter to evaluate $X$ and $\bar{\varepsilon}_{0.5}$ 
$c_{m d y n}$ MDRX parameter to evaluate $X$ and $t_{0.5}$

$d_{a}^{s s} \quad$ Steady state grain size when recrystallization is $100 \%$ for any grain family represented by $a$

$d_{0} \quad$ Initial grain size used in evaluating the microstructure variables

$d_{1 d y n}$ Temperature specific DRX parameter to evaluate $X$ and $\bar{\varepsilon}_{0.5}$

$d_{1 m d y n}$ MDRX parameter to evaluate $X$ and $t_{0.5}$

$d_{2 d y n}$ Temperature specific DRX parameter to evaluate $X$ and $\bar{\varepsilon}_{0.5}$

$d_{2 m d y n}$ MDRX parameter to evaluate $X$ and $t_{0.5}$

$d_{d y n} \quad$ Temperature specific DRX parameter to evaluate $X$ and $\bar{\varepsilon}_{0.5}$

$d_{g g r} \quad$ Grain size during static grain growth

$d_{\text {rexrex }}$ Instantaneous recrystallized grain size evaluated using the equations for the recrystallized grain family

$d_{\text {rexst }}$ Instantaneous strained grain size evaluated using the equations for the recrystallized grain family

$d_{\text {strex }}$ Instantaneous recrystallized grain size evaluated using the equations for the strained grain family

$d_{\text {stst }}$ Instantaneous strained grain size evaluated using the equations for the strained grain family

$f_{d y n}$ Temperature specific DRX parameter to evaluate $\bar{\varepsilon}_{p}$

$f_{m d y n}$ MDRX parameters to evaluate $X$ and $t_{0.5}$

$g_{d y n}$ Temperature specific DRX parameter to evaluate $\bar{\varepsilon}_{p}$

$h_{e} \quad$ Film coefficient of the fluid

$h_{1 d y n}$ Temperature specific DRX parameter to evaluate $\bar{\varepsilon}_{p}$ 
$h_{2 d y n}$ Temperature specific DRX parameter to evaluate $\bar{\varepsilon}_{p}$

$k \quad$ Thermal conductivity

$m \quad$ Exponent

$n_{i} \quad$ Component of the unit vector

$n_{\alpha} \quad$ Exponent for calculating the strained grain size

$n_{\text {dynctop }}$ Fraction to calculate the critical strain $\bar{\varepsilon}_{c}$

$n_{g g r}$ Exponent denoting the type of grain growth law (quadratic or cubic)

$n_{x d r x s t}$ Exponent to calculate the instantaneous strained grain size due to DRX

$n_{x d r x}$ Exponent to calculate the instantaneous grain size due to DRX

$n_{\text {xmdrxst }}$ Exponent to calculate the instantaneous strained grain size due to MDRX

$n_{x m d r x}$ Exponent to calculate the instantaneous grain size due to MDRX

nel Total number of elements

p Hydrostatic pressure

$p_{m d y n}$ MDRX parameter to evaluate steady state grain size

$q_{h} \quad$ Heat flux due to convection

$q_{r} \quad$ Heat flux due to radiation

$q_{1 m d y n}$ MDRX parameter to evaluate steady state grain size

$q_{2 m d y n}$ MDRX parameter to evaluate steady state grain size

$r_{m d y n}$ MDRX parameter to evaluate steady state grain size

$s_{m d y n}$ MDRX parameter to evaluate steady state grain size

$t_{0.5}$ Time required to achieve $50 \%$ recrystallization due to MDRX 
$t_{g g r}$ Grain growth parameter which is material specific

$t_{v} \quad$ Virtual time

$u_{i} \quad$ Velocity component

$u_{x, y, z}$ Velocity components

$x_{i} \quad$ Component of the displacement vector 


\section{CHAPTER 1: INTRODUCTION}

\subsection{Background}

Superalloys are metallic alloys used for high temperature $\left(>650{ }^{\circ} \mathrm{C}\right)$ applications such as encountered in the aircraft industry and where resistance to deformation is a primary requirement. Nickel-base alloys such as waspaloy and Alloy 718 (IN 718) are examples of superalloys that resist deformation at elevated temperatures and are therefore difficult to hot work. Hot working is the term often used to describe the plastic deformation at temperatures high enough to overcome strain hardening. The major hotworking operations are open-die press forging, radial forging (such as GFM) [2], extrusion, and rolling. In the case of rolling and forging, there may be many passes and some reheats involved. The hotworking operation under consideration is the continuous shape rolling process. In this process, billets transform from round-tooval and oval-to-round until the desired shape and size are obtained in multiple stands. The terms multi-stand and multi-pass will be interchangeably used throughout this thesis to denote the continuous rolling process.

For a given composition of alloy, the high temperature flow stress is influenced to a large extent by the grain size of the microstructure. In the case of rolling, the correct working forces, which relate to gauge and shape control as well as to power requirements, can be estimated accurately only if the microstructure relevant to the specific pass of rolling is known. The microstructure present at the end of the rolling and cooling operations also controls the product properties. Coarser grain size favors creep strength and crack-growth resistance while a fine grain structure favors improved low-cycle fatigue life and tensile yield strength. Control of grain size is an increasingly important characteristic in any hot-working due to the stringent

ultrasonic inspectability requirements [44]. The temperature range for hot working of waspaloy is $\approx 975{ }^{\circ} \mathrm{C}-1175{ }^{\circ} \mathrm{C}$ [44] and for Alloy 718 is $\approx 980{ }^{\circ} \mathrm{C}-1120{ }^{\circ} \mathrm{C}$ 
[44; 45; 70] depending on the final microstructure desired. This narrow temperature range is due to the increase in the percentages of titanium and aluminum [45; 49]) makes the grain size control more difficult. Alloy 718 and its derivatives are the main focus of the current work and capturing the microstructural changes due to deformation during a multistand continuous rolling forms the core objective of this work.

\subsection{Motivation}

Modeling the dynamic microstructural events is important for determining flow stress levels, and hence rolling loads. Tremendous amount of time and effort is needed in carrying out experiments and establishing the constitutive models for the various recrystallization processes. In addition, industrial trials are expensive, difficult to control, monitor and sample accurately, and necessarily constrained within the capabilities of the existing plant. Laboratory simulation tests are unable to reproduce all the conditions of industrial multi-pass rolling. There are limitations on strain rates attainable, particularly in relation to finish strip and rod rolling. An alternative and simpler approach employing plane strain compression testing cannot achieve the total strains of complete industrial rolling schedules. Therefore, numerical methods are resorted to to study the influence of the vast number of variables present in a typical industrial multi-pass rolling on the mictrostructure [29]. With the growth in the application of computers and their capabilities, numerical techniques have become quite common in solving plastic deformation problems. One of the popular numerical techniques is the Finite Element (FE) Method. Process variables such as strain and temperature are predicted from $\mathrm{FE}$ analysis of the deformation process. In general, microstructural modeling relates those process variables to microstructural evolution. Typical microstructural modeling involves two major steps. In the first step, constitutive equations describing the microstructural evolutions are developed using experiments. In the second step, the microstructural constitutive equations are implemented in a commercial FE package such as ABAQUS [38] or a custom-built software. In the present work, we focus on the latter, i.e., on the implementation of microstructural models in a custom-built software called RAWHIDE [1]. 


\subsection{Overview}

A brief discussion of the observed microstructural evolution processes and an overview of the existing FE package (RAWHIDE) are given in the following sections.

\subsubsection{Microstructural processes}

During hotworking, materials undergo recovery and recrystallization processes that can be dynamic or static [94]. Recovery and recrystallization are competing processes and are driven by the stored energy of the deformed state. The extent of recovery depends on recrystallization. If deformation substructure is consumed due to recrystallization, no further recovery will occur. Recrystallization can be continuous or discontinuous [61]. There are dynamic, metadynamic and static recrystallizations depending on the strains and dislocation densities. Dynamic recrystallization (DRX) occurs during the actual deformation, when the equivalent strain exceeds a certain critical strain. During metadynamic recrystallization (MDRX), the partially recrystallized grain structure observed right after deformation transforms to a more fully recrystallized structure. It takes place by the growth of recrystallization nuclei formed during dynamic recrystallization. MDRX involves imposed strains greater than the critical strain. Static recrystallization (SRX) occurs after deformation in which the imposed strain is less than the critical strain. Recrystallization begins in a nuclei-free environment [45]. A fine-grained structure is generated primarily during dynamic and static recrystallization. Significant static recrystallization can take place during reheating. SRX is not considered in the current work. Alloy 718 is governed by the fcc lattice structure of the $\gamma$ matrix [70] and a number of characteristic precipitates such as $\gamma^{\prime \prime}(\mathrm{Ni3}(\mathrm{Nb}, \mathrm{Ti})), \gamma^{\prime}, \delta$, and carbides (MC and M6C). The high temperature strength of Alloy 718 is derived essentially from the coherent $\gamma^{\prime \prime}$ and to a smaller extent from $\gamma^{\prime}$. The presence of the other precipitates improves hot working to produce very fine-grained billet structures. However, modeling the complex precipitation processes requires a more detailed understanding of precipitation kinetics, than is presently available. Therefore, the current work aims to find the average grain sizes from recrystallization processes alone and does not take into account the precipitation of phases. 


\subsubsection{FE package RAWHIDE}

RAWHIDE is a custom-built FE package specifically developed to analyze the multi-pass shape rolling. RAWHIDE models the multi-pass rolling as a steady state creeping flow problem wherein the viscosity of the fluid is a function of temperature, effective strain rate and effective strain. RAWHIDE encompasses various numerical algorithms such as conjugate gradient method and an approach that determines the contact point between the roll surface and the free surface of the material being rolled. The details are discussed in Chapter 2. Typically, the results from a RAWHIDE analysis are the process variables such as the velocity components and temperature. In this analysis, the heat generated during plastic deformation is incorporated as a bulk quantity which is a fraction of the plastic work. Between the stands, a two-dimensional heat conduction problem is solved in order to capture the temperature changes in the bar. RAWHIDE is numerically efficient, fast and can be used in designing rolling schedules and analyzing process anomalies. However, a typical multi-pass rolling process consists of as many as 26 stands and as the workpiece passes through these stands, the material deforms significantly and consequently, mesh dis-

tortion becomes progressively severe. The algorithm presented in [1] does not have remeshing capabilities.

\subsection{Objectives}

It is apparent from the foregoing discussions that the major objective of the current research is to model the microstructural evolution during multi-pass rolling. There are also computational issues that require attention in order to model the microstructure accurately over many stands. Specifically, RAWHIDE requires some modifications. The scope of the current work involves the following objectives:

- Develop and incorporate mesh re-zoning algorithm into RAWHIDE. Mesh rezoning is a collective term describing remeshing and transfer of data from the original distorted mesh to the newly created mesh from remeshing.

- Modify RAWHIDE as necessary to predict the microstructure evolution as rolling proceeds. 
- Develop a microstructural modeling algorithm for multi-pass rolling and validate specifically for Alloy 718.

- Validate the microstructure modeling algorithm by comparing the microstructure predicted by employing the algorithm with experimental results for Alloy 718.

\subsection{Dissertation organization}

The dissertation is organized in such a way that it meets the objectives of the research.

In Chapter 2, a detailed discussion of the formulation behind RAWHIDE is given. A detailed understanding of RAWHIDE forms the basis for the solution procedure developed in Chapter 3.

In Chapter 3, a brief literature survey on mesh re-zoning procedures, and the proposed approach and results to address the mesh distortion issue are explained.

In Chapter 4, a comprehensive explanation of the fundamentals of microstructural processes is given. In addition, the outcome of an extensive literature survey on the existing microstructure prediction approaches are given in this Chapter. This Chapter lays the foundation for the proposed microstructure prediction approach of the current work.

In Chapter 5, the proposed microstructure prediction algorithm is presented and its numerical implementation is explained.

In Chapter 6, results from the developed procedure in the context of a rolling analysis for a specific material are presented and discussed. A comparison of the numerical results with the experimental observation for Alloy 718 is also given.

In Chapter 7, a summary of the present work, conclusions and the scope for future work is explored. 


\section{CHAPTER 2: FINITE ELEMENT FORMULATION}

\subsection{RAWHIDE Introduction}

The multi-pass rolling considered in the current research is simulated using a custom-built FE code known as RAWHIDE for modeling rolling problems. In this code, the material behavior is modeled as that of an incompressible, non-Newtonian fluid. Three dimensional steady-state thermomechanical analysis is used during each pass and transient thermal analysis for cooling is used between passes. RAWHIDE incorporates the methodology developed by Thompson, et al., [1] that adopts a mixedmethod formulation originally proposed by Zienkiewicz, et al., [4]. In this formulation, the locations of nodes at steady-state in each stand are obtained by an iterative approach using a modified Euler integration of the current velocity field [9; 10].

The flow field of a system can be influenced by the thermal response since the viscosity is a function of temperature. On the other hand, the temperature field is coupled to the flow field by viscous dissipation due to plastic straining and by the transport of heat associated with moving material. The governing momentum and energy balance equations are solved iteratively to find the velocity components and temperature at each node in the three-dimensional FE mesh while satisfying the incompressibility constraint simultaneously. The procedure for this coupling is schematically represented in FIGURE 2.1, which is based on references [13] and [14].

The formulation that formed the basis for developing RAWHIDE is presented in detail in this Chapter. A deep understanding of RAWHIDE is necessary in order to modify this package. Some improvements to this custom-built package are necessary for predicting microstructure for many stands. The details of the improvements are discussed in the next Chapter. The current discussion is limited only to the formulation and the capabilities of RAWHIDE without the proposed modifications. Firstly, the variational formulation of the flow problem is discussed followed by the formulation of the heat transfer problem. A brief discussion of the mesh generation and 


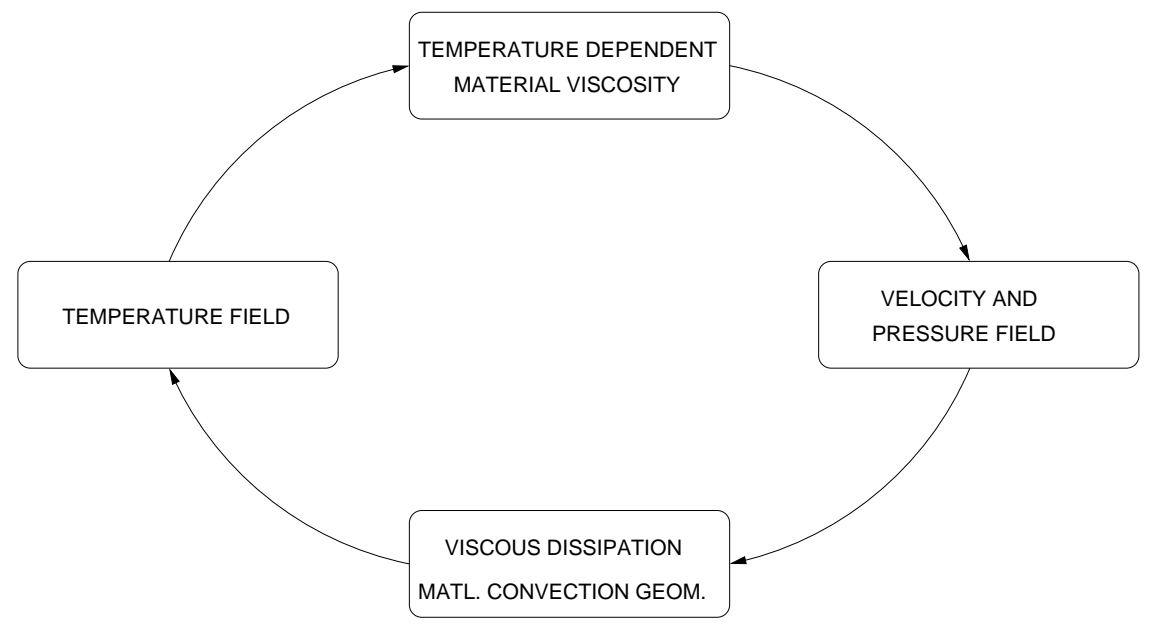

FIGURE 2.1: Thermo-mechanical coupling.

various results from RAWHIDE are discussed in the end.

\subsection{Variational flow formulation}

Large casting made of metallic materials are subjected to processes such as rolling, forging, and extrusion in the solid state. These processes are termed as thermomechanical processes in which, the material may either be hot or cold, and may involve intermediate anneals [94]. RAWHIDE considers the rolled material to assume incompressible viscous laminar flow $(R e<<1)$ without inertia. This flow is sometimes represented as laminar creeping flow or Stoke's flow. There are two approaches to solve such a problem.

1. Stream function formulation.

2. Velocity and pressure formulation.

The first approach requires $C^{1}$ continuity which is difficult to achieve and accordingly is disadvantageous to use. The second approach involves two variables namely velocity and pressure, where velocity is a primary variable and pressure is a constraint variable.

Consider the potential energy functional [12],

$$
J=\int_{\Omega} W d \Omega-\int_{\Gamma_{t}} \bar{T}_{i} u_{i} d \Gamma-\int_{\Omega} \rho X_{i} u_{i} d \Omega,
$$


where $\mathrm{W}$ is related to $\dot{\varepsilon}_{i j}^{\prime}$ as follows:

$$
\frac{\partial W}{\partial \dot{\varepsilon}_{i j}^{\prime}}=\sigma_{i j}^{\prime}
$$

Upon substituting equation (2.2) in equation (2.1) the functional takes the form,

$$
J=\int_{\Omega} \sigma_{i j}^{\prime} \dot{\varepsilon}_{i j}^{\prime} d \Omega-\int_{\Gamma_{t}} \bar{T}_{i} u_{i} d \Gamma-\int_{\Omega} \rho X_{i} u_{i} d \Omega
$$

Also, note that $\sigma_{i j}^{\prime} \delta \dot{\varepsilon}_{i j}^{\prime}=\sigma_{i j}^{\prime} \delta \dot{\varepsilon}_{i j}$ since $\sigma_{i i}^{\prime}=0$. Therefore,

$$
J=\int_{\Omega} \sigma_{i j}^{\prime} \dot{\varepsilon}_{i j} d \Omega-\int_{\Gamma_{t}} \bar{T}_{i} u_{i} d \Gamma-\int_{\Omega} \rho X_{i} u_{i} d \Omega
$$

In the above equation (2.4), the first term represents the rate of internal work, second term represents the rate of external work due to prescribed traction and the third term, the rate of external work due to prescribed body forces.

Hermann [8] describes the variational principle to include the constraint of incompressibility into potential energy functional (if inertial effects are negligible). Accordingly, introducing an auxiliary condition using a Lagrange multiplier, that is equivalent to the incompressibility constraint on the velocity field [6], equation (2.4) takes the form,

$$
J^{*}=\int_{\Omega} \sigma_{i j}^{\prime} \dot{\varepsilon}_{i j} d \Omega-\int_{\Gamma_{t}} \bar{T}_{i} u_{i} d \Gamma-\int_{\Omega} \rho X_{i} u_{i} d \Omega+\int_{\Omega} \lambda \dot{\varepsilon}_{i i} d \Omega
$$

The class of admissible functions can now be extended to compressible as well as incompressible materials. The first variation of the functional in equation (2.5) is given by,

$$
\begin{aligned}
\delta J^{*} & =\int_{\Omega} \sigma_{i j}^{\prime} \delta \dot{\varepsilon}_{i j} d \Omega-\int_{\Gamma_{t}} \bar{T}_{i} \delta u_{i} d \Gamma-\int_{\Omega} \rho X_{i} \delta u_{i} d \Omega+\int_{\Omega} \lambda \delta \dot{\varepsilon}_{i i} d \Omega+\int_{\Omega} \delta \lambda \dot{\varepsilon}_{i i} d \Omega \\
& =\int_{\Omega}\left(\sigma_{i j}^{\prime}+\lambda \delta_{i j}\right) \delta \dot{\varepsilon}_{i j} d \Omega-\int_{\Gamma_{t}} \bar{T}_{i} \delta u_{i} d \Gamma-\int_{\Omega} \rho X_{i} \delta u_{i} d \Omega+\int_{\Omega} \delta \lambda \dot{\varepsilon}_{i i} d \Omega,
\end{aligned}
$$


where the differential strain rate is given by,

$$
\delta \dot{\varepsilon}_{i j}=\frac{1}{2}\left[\frac{\partial\left(\delta u_{i}\right)}{\partial x_{j}}+\frac{\partial\left(\delta u_{j}\right)}{\partial x_{i}}\right]
$$

In equation (2.6), consider the first integral as $I_{1}$. That is,

$$
I_{1}=\int_{\Omega}\left(\sigma_{i j}^{\prime}+\lambda \delta_{i j}\right) \delta \dot{\varepsilon}_{i j} d \Omega
$$

Substituting equation (2.7) in the above equation and using the chain rule of integration,

$$
I_{1}=\frac{1}{2} \int_{\Omega}\left(\sigma_{i j}^{\prime}+\lambda \delta_{i j}\right) \frac{\partial\left(\delta u_{i}\right)}{\partial x_{j}} d \Omega+\frac{1}{2} \int_{\Omega}\left(\sigma_{i j}^{\prime}+\lambda \delta_{i j}\right) \frac{\partial\left(\delta u_{j}\right)}{\partial x_{i}} d \Omega .
$$

Expanding the terms in the above equation results in,

$$
\begin{aligned}
I_{1}=\frac{1}{2} \int_{\Omega} \frac{\partial}{\partial x_{j}} & {\left[\left(\sigma_{i j}^{\prime}+\lambda \delta_{i j}\right) \delta u_{i}\right] d \Omega-\frac{1}{2} \int_{\Omega} \frac{\partial}{\partial x_{j}}\left[\left(\sigma_{i j}^{\prime}+\lambda \delta_{i j}\right)\right] \delta u_{i} d \Omega } \\
& +\frac{1}{2} \int_{\Omega} \frac{\partial}{\partial x_{i}}\left[\left(\sigma_{i j}^{\prime}+\lambda \delta_{i j}\right) \delta u_{j}\right] d \Omega-\frac{1}{2} \int_{\Omega} \frac{\partial}{\partial x_{i}}\left[\left(\sigma_{i j}^{\prime}+\lambda \delta_{i j}\right)\right] \delta u_{j} d \Omega
\end{aligned}
$$

Since the deviatoric stress $\boldsymbol{\sigma}^{\prime}$ and identity tensor $\mathbf{I}$ are both symmetric, like terms add up in equation (2.8) and therefore equation (2.6) becomes,

$$
\begin{aligned}
\delta J^{*}=\int_{\Omega}\left\{\frac{\partial}{\partial x_{j}}\left[\left(\sigma_{i j}^{\prime}+\lambda \delta_{i j}\right) \delta u_{i}\right]\right. & \left.-\frac{\partial}{\partial x_{j}}\left[\left(\sigma_{i j}^{\prime}+\lambda \delta_{i j}\right)\right] \delta u_{i}\right\} d \Omega \\
& -\int_{\Gamma_{t}} \bar{T}_{i} \delta u_{i} d \Gamma-\int_{\Omega} \rho X_{i} \delta u_{i} d \Omega+\int_{\Omega} \delta \lambda \dot{\varepsilon}_{i i} d \Omega
\end{aligned}
$$

Using Gauss's theorem, the first term within the parentheses in the above integral can be written as,

$$
\int_{\Omega} \frac{\partial}{\partial x_{j}}\left[\left(\sigma_{i j}^{\prime}+\lambda \delta_{i j}\right) \delta u_{i}\right] d \Omega=\int_{\Gamma_{t}}\left(\sigma_{i j}^{\prime}+\lambda \delta_{i j}\right) n_{j} \delta u_{i} d \Gamma+\int_{\Gamma_{u}}\left(\sigma_{i j}^{\prime}+\lambda \delta_{i j}\right) n_{j} \delta u_{i} d \Gamma
$$


Substituting equation (2.10) in equation (2.9),

$$
\begin{aligned}
\delta J^{*}=-\int_{\Omega}\left\{\frac{\partial}{\partial x_{j}}\left(\sigma_{i j}^{\prime}+\lambda \delta_{i j}\right)\right. & \left.+\rho X_{i}\right\} \delta u_{i} d \Omega+\int_{\Gamma_{t}}\left\{\left(\sigma_{i j}^{\prime}+\lambda \delta_{i j}\right) n_{j}-\bar{T}_{i}\right\} \delta u_{i} d \Gamma \\
& +\int_{\Omega} \delta \lambda \dot{\varepsilon}_{i i} d \Omega
\end{aligned}
$$

The surface integral on boundary $\Gamma_{u}$, where velocity is specified has been ignored since on this boundary $\delta u_{i}=0$. In order to attain a stationary value, the first variation must vanish for any arbitrary $\delta u_{i}$ and $\delta \lambda$, and hence the following Euler equations are obtained.

$$
\begin{gathered}
\text { (i) } \frac{\partial}{\partial x_{j}}\left(\sigma_{i j}^{\prime}+\lambda \delta_{i j}\right)+\rho X_{i}=0, \quad \text { or, } \\
\sigma_{i j, j}+\rho X_{i}=0 \quad \text { in } \Omega . \\
(i i) \quad \frac{\partial u_{i}}{\partial x_{i}}=\dot{\varepsilon}_{i i}=0 \quad \text { in } \Omega . \\
\left(\sigma_{i j}^{\prime}+\lambda \delta_{i j}\right) n_{j}-\bar{T}_{i}=0, \quad \text { or } \\
\sigma_{i j} n_{j}=\bar{T}_{i} \quad \text { on } \quad \Gamma_{t} .
\end{gathered}
$$

In the above, it is apparent that the Lagrange multiplier is nothing but the hydrostatic pressure ' $-p$ ' since,

$$
\boldsymbol{\sigma}^{\prime}=\boldsymbol{\sigma}+p \mathbf{I}
$$

or,

$$
\boldsymbol{\sigma}=\boldsymbol{\sigma}^{\prime}-p \mathbf{I}
$$

The indicial form of the above equation $(2.15)$ is,

$$
\sigma_{i j}=\sigma_{i j}^{\prime}-p \delta_{i j}
$$

where, $p=-\sigma_{i i} / 3$. Therefore, the equivalence between the modified variational principle and boundary-value problem has been shown. 


\subsubsection{Field equations}

In the following various governing equations for an incompressible creeping viscous laminar flow are described. Firstly, the equations of motion, that is, the momentum balance equations are given. The linear momentum balance due to Navier-Stoke's equations without inertial terms is given as,

$$
\boldsymbol{\nabla} \cdot \boldsymbol{\sigma}+\mathbf{b}=\mathbf{0} \quad \text { in } \Omega
$$

or,

$$
\frac{\partial \sigma_{i j}}{\partial x_{j}}+\rho X_{i}=0 \quad \text { in } \quad \Omega .
$$

In the absence of body forces, equation (2.16) is given by,

$$
\frac{\partial \sigma_{i j}}{\partial x_{j}}=0 .
$$

The angular momentum balance is given by,

$$
\boldsymbol{\sigma}=\boldsymbol{\sigma}^{T} .
$$

The incompressibility condition is expressed as,

$$
\boldsymbol{\nabla} \cdot \mathbf{u}=0 \text { in } \Omega
$$

or,

$$
\frac{\partial u_{i}}{\partial x_{i}}=\dot{\varepsilon}_{i i}=0 \quad \text { in } \quad \Omega .
$$

The constitutive equation describing the behavior of the material being rolled is given by,

$$
\begin{aligned}
\boldsymbol{\sigma} & =2 \mu\left(\dot{\boldsymbol{\varepsilon}}-\frac{1}{3}(\dot{\boldsymbol{\varepsilon}}: \mathbf{I}) \mathbf{I}\right)-p \mathbf{I} \\
& =2 \mu\left(\dot{\boldsymbol{\varepsilon}}-\frac{1}{3} \dot{\varepsilon}_{i i} \mathbf{I}\right)-p \mathbf{I} .
\end{aligned}
$$


Due to incompressibility condition, the above equation can be expressed as,

$$
\boldsymbol{\sigma}=2 \mu \dot{\varepsilon}-p \mathbf{I}
$$

In indicial notation the above equation (2.17) takes the form,

$$
\sigma_{i j}=2 \mu \dot{\varepsilon}_{i j}-p \delta i j
$$

Applying equation (2.15) in equation (2.18) results in,

$$
\sigma_{i j}^{\prime}=2 \mu \dot{\varepsilon}_{i j}
$$

The term denoted by the symbol $\mu$ is the viscosity of the fluid. The viscosity follows Bingham's law described by the following equation for viscoplastic metals [5],

$$
\mu=\frac{\sigma_{y}+\tau \dot{\bar{\varepsilon}}^{m}}{3 \dot{\bar{\varepsilon}}} .
$$

For ideal plastic materials $\tau=0$, resulting in,

$$
\mu=\frac{\sigma_{y}}{3 \dot{\bar{\varepsilon}}}
$$

Flow stress is a function of all hot-deformation parameters. Alloy 718 shows the existence of dynamic softening, that is, the flow stress decreases when temperature increases [65]. Work hardening plays a key role in the deformation of superalloy Alloy 718 at small strains. Dynamic softening dominates in the deformation of Alloy 718 when the strain is sufficiently large. Strain rate dependence is also observed. Therefore, in the case of Alloy 718, the flow stress is a function of effective strain, temperature, and effective strain rate $(\dot{\bar{\varepsilon}})$. RAWHIDE models the viscosity as the function of these parameters while the flow stress is a function of the viscosity. That is,

$$
\sigma_{y}=\sigma_{y}(\bar{\varepsilon}, \dot{\bar{\varepsilon}}, T)
$$


and hence,

$$
\mu=\mu(\bar{\varepsilon}, \dot{\bar{\varepsilon}}, T)
$$

which makes the equations non-linear. The boundary conditions for the analysis are given by,

$$
\text { (a) } \quad \sigma_{i j} n_{j}=\bar{T}_{i} \quad \text { on } \quad \Gamma_{t}
$$

and

$$
\text { (b) } u_{i}=\bar{u}_{i} \text { on } \Gamma_{u}
$$

The other relationships that are incorporated in RAWHIDE in determining some of the input variables necessary for solving the governing equations and postprocessing of the results are given in the following discussion. The effective stress and effective strain rates are given as,

$$
\begin{aligned}
\bar{\sigma}^{2} & =\frac{3}{2} \boldsymbol{\sigma}^{\prime}: \boldsymbol{\sigma}^{\prime} \\
& =\frac{3}{2} \sigma_{i j}^{\prime} \sigma_{i j}^{\prime} \\
\dot{\bar{\varepsilon}}^{2} & =\frac{2}{3} \dot{\varepsilon}: \dot{\varepsilon} \\
& =\frac{2}{3} \dot{\varepsilon}_{i j} \dot{\varepsilon}_{i j}
\end{aligned}
$$

that is,

$$
\dot{\bar{\varepsilon}}=\sqrt{\left.\frac{2}{3}\left\{\left(\dot{\varepsilon}_{x x}^{2}+\dot{\varepsilon}_{y y}^{2}+\dot{\varepsilon}_{z z}^{2}\right)+2\left(\dot{\varepsilon}_{x y}^{2}+\dot{\varepsilon}_{x z}^{2}+\dot{\varepsilon}_{y z}^{2}\right)\right)\right\}} .
$$

Using the constitutive equation (2.19), the effective stress can be written as,

$$
\begin{aligned}
\bar{\sigma}^{2} & =\frac{3}{2} \cdot 2 \mu \dot{\varepsilon}: 2 \mu \dot{\varepsilon} \\
& =6 \mu^{2} \dot{\varepsilon}: \dot{\varepsilon} \\
& =6 \mu^{2} \frac{3}{2} \dot{\bar{\varepsilon}}^{2}
\end{aligned}
$$

Therefore,

$$
\bar{\sigma}=3 \mu \dot{\bar{\varepsilon}}
$$


The plastic work is given by,

$$
\begin{aligned}
W & =\boldsymbol{\sigma}^{\prime}: \dot{\boldsymbol{\varepsilon}} \\
& =\sigma_{i j}^{\prime} \dot{\varepsilon}_{i j} .
\end{aligned}
$$

Upon substituting equation (2.19) and equation (2.21) in the equation (2.23) results in,

$$
\begin{aligned}
W & =2 \mu \frac{3}{2} \dot{\bar{\varepsilon}}^{2} \\
& =3 \mu \dot{\bar{\varepsilon}}^{2}
\end{aligned}
$$

Accordingly, the heat generation rate due to plastic work is,

$$
\dot{Q}=\beta W
$$

where $\beta$ is the work to heat ratio, also known as the Taylor-Quinney coefficient. The work to heat ratio is specified as a material property varying between 0.9 and 0.95 .

2.3 FE flow formulation

Assuming no body forces and substituting the constitutive equation (2.19), the equation 2.5 can be rewritten as,

$$
\begin{aligned}
\delta J^{*} & =\int_{\Omega} \sigma_{i j}^{\prime} \delta \dot{\varepsilon}_{i j} d \Omega-\int_{\Gamma_{t}} \bar{T}_{i} \delta u_{i} d \Gamma+\int_{\Omega} p \delta \dot{\varepsilon}_{i i} d \Omega+\int_{\Omega} \delta p \dot{\varepsilon}_{i i} d \Omega \\
& =\int_{\Omega} \dot{\varepsilon}_{i j} 2 \mu \delta \dot{\varepsilon}_{i j} d \Omega-\int_{\Gamma_{t}} \bar{T}_{i} \delta u_{i} d \Gamma+\int_{\Omega} p \delta \dot{\varepsilon}_{i i} d \Omega+\int_{\Omega} \delta p \dot{\varepsilon}_{i i} d \Omega
\end{aligned}
$$

In the above expression, the Lagrange multiplier was replaced by the hydrostatic pressure as discussed earlier. 


\subsubsection{Matrix form}

Let the terms strain rate $(\dot{\boldsymbol{\varepsilon}})$, viscosity $(\boldsymbol{D})$, traction vector $(\bar{T})$, and velocity $(\boldsymbol{u})$ be defined in matrix form as follows:

$$
\begin{gathered}
\dot{\boldsymbol{\varepsilon}}=\{\dot{\boldsymbol{\varepsilon}}\}=\left\{\begin{array}{c}
\dot{\varepsilon}_{x x} \\
\dot{\varepsilon}_{y y} \\
\dot{\varepsilon}_{z z} \\
\dot{\varepsilon}_{x y} \\
\dot{\varepsilon}_{x z} \\
\dot{\varepsilon}_{y x}
\end{array}\right\},[\boldsymbol{D}]=\left[\begin{array}{cccccc}
2 \mu & 0 & 0 & 0 & 0 & 0 \\
0 & 2 \mu & 0 & 0 & 0 & 0 \\
0 & 0 & 2 \mu & 0 & 0 & 0 \\
0 & 0 & 0 & 4 \mu & 0 & 0 \\
0 & 0 & 0 & 0 & 4 \mu & 0 \\
0 & 0 & 0 & 0 & 0 & 4 \mu
\end{array}\right], \\
\overline{\boldsymbol{T}}=\{\overline{\boldsymbol{T}}\}=\left\{\begin{array}{l}
\bar{T}_{x} \\
\bar{T}_{y} \\
\bar{T}_{z}
\end{array}\right\}, \boldsymbol{u}=\{\boldsymbol{u}\}=\left\{\begin{array}{c}
u_{x} \\
u_{y} \\
u_{z}
\end{array}\right\}, \text { and }\{\boldsymbol{m}\}=\left\{\begin{array}{c}
1 \\
1 \\
1 \\
0 \\
0
\end{array}\right\} .
\end{gathered}
$$

The variation of the functional for a single element in matrix form from equation (2.25) is therefore,

$$
\begin{aligned}
\delta J_{e}^{*}=\int_{\Omega^{e}}\{\delta \dot{\boldsymbol{\varepsilon}}\}^{T}[\boldsymbol{D}]\{\dot{\boldsymbol{\varepsilon}}\} d \Omega^{e} & -\int_{\Gamma_{t}^{e}}\{\delta \boldsymbol{u}\}^{T}\{\overline{\boldsymbol{T}}\} d \Gamma^{e}-\int_{\Omega^{e}}\{\delta \dot{\boldsymbol{\varepsilon}}\}^{T}\{\boldsymbol{m}\} p d \Omega^{e} \\
& -\int_{\Omega^{e}} \delta p\{\boldsymbol{m}\}^{T}\{\dot{\boldsymbol{\varepsilon}}\} d \Omega^{e}
\end{aligned}
$$

where, $\dot{\varepsilon}_{i i}=\{\boldsymbol{m}\}^{T}\{\dot{\boldsymbol{\varepsilon}}\}$, and $\boldsymbol{\sigma}=[\boldsymbol{D}]\{\dot{\boldsymbol{\varepsilon}}\}$.

\subsubsection{Galerkin's formulation}

Galerkin's procedure is used to derive the discretized finite element formulation by defining a trilinear solid brick element. 
Therefore,

$$
\left\{\begin{array}{c}
u_{x}(x, y, z) \\
u_{y}(x, y, z) \\
u_{z}(x, y, z)
\end{array}\right\}=\left[\begin{array}{cccccccccc}
N_{1} & 0 & 0 & N_{2} & 0 & 0 & \cdots & N_{8} & 0 & 0 \\
0 & N_{1} & 0 & 0 & N_{2} & 0 & \cdots & N_{8} & 0 & 0 \\
0 & 0 & N_{1} & 0 & 0 & N_{2} & \cdots & N_{8} & 0 & 0
\end{array}\right]\left\{\begin{array}{c}
u_{1 x} \\
u_{1 y} \\
u_{1 z} \\
\vdots \\
u_{8 x} \\
u_{8 y} \\
u_{8 z}
\end{array}\right\}
$$

or,

$$
\{\boldsymbol{u}(x, y, z)\}^{e}=\left[\boldsymbol{N}^{u}\right]^{e}\{\hat{\boldsymbol{u}}\}^{e}
$$

That is,

$$
\{\boldsymbol{u}(\boldsymbol{x})\}^{e}=\left[\boldsymbol{N}^{u}\right]^{e}\{\hat{\boldsymbol{u}}\}^{e}
$$

Similarly,

$$
p(\boldsymbol{x})^{e}=\left[\boldsymbol{N}^{p}\right]^{e}\{\hat{\boldsymbol{p}}\}^{e} .
$$

Now, the strain rate within an element is,

$$
\{\dot{\boldsymbol{\varepsilon}}\}^{e}=\left[\boldsymbol{N}^{\prime \prime}\right]^{e}\{\hat{\boldsymbol{u}}\}^{e},
$$

or,

$$
\left\{\begin{array}{c}
\dot{\varepsilon}_{x x}(\boldsymbol{x}) \\
\dot{\varepsilon}_{y y}(\boldsymbol{x}) \\
\dot{\varepsilon}_{z z}(\boldsymbol{x}) \\
\dot{\varepsilon}_{x y}(\boldsymbol{x}) \\
\dot{\varepsilon}_{x z}(\boldsymbol{x}) \\
\dot{\varepsilon}_{y x}(\boldsymbol{x})
\end{array}\right\}=\left[\begin{array}{cccccccccc}
\frac{\partial N_{1}}{\partial x} & 0 & 0 & \frac{\partial N_{2}}{\partial x} & 0 & 0 & \ldots & \frac{\partial N_{8}}{\partial x} & 0 & 0 \\
0 & \frac{\partial N_{1}}{\partial y} & 0 & 0 & \frac{\partial N_{2}}{\partial y} & 0 & \ldots & \frac{\partial N_{8}}{\partial y} & 0 & 0 \\
0 & 0 & \frac{\partial N_{1}}{\partial z} & 0 & 0 & \frac{\partial N_{2}}{\partial z} & \ldots & \frac{\partial N_{8}}{\partial z} & 0 & 0 \\
\frac{\partial N_{1}}{2 \partial y} & \frac{\partial N_{1}}{2 \partial x} & 0 & \frac{\partial N_{2}}{2 \partial y} & \frac{\partial N_{2}}{2 \partial x} & 0 & \ldots & \frac{\partial N_{8}}{2 \partial y} & \frac{\partial N_{8}}{2 \partial x} & 0 \\
\frac{\partial N_{1}}{2 \partial z} & 0 & \frac{\partial N_{1}}{2 \partial x} & \frac{\partial N_{2}}{2 \partial z} & 0 & \frac{\partial N_{2}}{2 \partial x} & \cdots & \frac{\partial N_{8}}{2 \partial z} & 0 & \frac{\partial N_{8}}{2 \partial x} \\
0 & \frac{\partial N_{1}}{2 \partial z} & \frac{\partial N_{1}}{2 \partial y} & 0 & \frac{\partial N_{2}}{2 \partial z} & \frac{\partial N_{2}}{2 \partial y} & \cdots & 0 & \frac{\partial N_{8}}{2 \partial z} & \frac{\partial N_{8}}{2 \partial y}
\end{array}\right]\left\{\begin{array}{c}
u_{1 x} \\
u_{1 y} \\
u_{1 z} \\
\vdots \\
u_{8 x} \\
u_{8 y} \\
u_{8 z}
\end{array}\right\} .
$$


That is,

$$
\{\dot{\boldsymbol{\varepsilon}}\}^{e}=\left[\boldsymbol{B}^{u}\right]^{e}\{\hat{\boldsymbol{u}}\}^{e} .
$$

Using the approximating functions described before, the variation of the functional in the matrix form expressed in equation (2.26) for a single element can be written as,

$$
\delta J_{e}^{*}=\left\{\delta \boldsymbol{u}^{e}\right\}^{T}\left[\boldsymbol{k}_{d}^{e}\right]\left\{\boldsymbol{u}^{e}\right\}-\left\{\delta \boldsymbol{u}^{e}\right\}^{T}\left[\boldsymbol{q}^{e}\right]\left\{p^{e}\right\}-\left\{\delta p^{e}\right\}^{T}\left[\boldsymbol{q}^{e}\right]\left\{\boldsymbol{u}^{e}\right\}-\left\{\delta \boldsymbol{u}^{e}\right\}^{T}\left\{\boldsymbol{f}^{e}\right\}
$$

Using standard assembly procedures this can be written for the whole domain as,

$$
\delta J=\{\delta \boldsymbol{u}\}^{T}\left[\boldsymbol{K}_{D}\right]\{\boldsymbol{u}\}-\{\delta \boldsymbol{u}\}^{T}[\boldsymbol{Q}]\{\boldsymbol{p}\}-\{\delta \boldsymbol{p}\}^{T}[\boldsymbol{Q}]^{T}\{\boldsymbol{u}\}-\{\delta \boldsymbol{u}\}^{T}\{\boldsymbol{f}\}
$$

FE formulation of the above leads to,

$$
\left[\begin{array}{ll}
\mathbf{K}_{D} & \mathbf{Q} \\
\mathbf{Q}^{T} & \mathbf{0}
\end{array}\right]\left\{\begin{array}{l}
\mathbf{u} \\
\mathbf{p}
\end{array}\right\}=\left\{\begin{array}{l}
\mathbf{f} \\
\mathbf{0}
\end{array}\right\}
$$

where,

$$
\begin{aligned}
\mathbf{K}_{D} & =\underset{1}{\mathbf{A}} \int_{\Omega^{e}}\left[\mathbf{B}^{u}\right]^{T}[\mathbf{D}]\left[\mathbf{B}^{u}\right] d \Omega^{e}, \\
\mathbf{Q} & =\underset{1}{\mathbf{A}} \int_{\Omega^{e}}\left[\mathbf{B}^{u}\right]^{T}\{\mathbf{m}\}\left[\mathbf{N}^{p}\right] d \Omega^{e}, \text { and } \\
\mathbf{f} & =\underset{1}{\mathbf{n e l}} \int_{\Gamma_{t}^{e}}\left[\mathbf{N}^{u}\right]\{\bar{T}\} d \Gamma^{e} .
\end{aligned}
$$

In the equation set shown by (2.29), the first equation describes the variation of functional with reference to an arbitrary $\delta u$ while the second equation describes the variation of functional with respect to an arbitrary $\delta p$. This system augmented with a penalty function [4] can be written as,

$$
\left[\begin{array}{cc}
\mathbf{K}_{D} & \mathbf{Q} \\
\mathbf{Q}^{T} & -\frac{1}{\gamma} \mathbf{I}
\end{array}\right]\left\{\begin{array}{l}
\mathbf{u} \\
\mathbf{p}
\end{array}\right\}=\left\{\begin{array}{c}
\mathbf{f} \\
-\frac{1}{\gamma} \mathbf{p}
\end{array}\right\},
$$


and the solution can be found iteratively as follows for velocities and pressure,

$$
\begin{aligned}
\mathbf{p}^{n+1} & =\mathbf{p}^{n}+\gamma\left(\mathbf{Q}^{T} \mathbf{u}^{n}\right) \\
{\left[\mathbf{K}_{D}+\gamma\left(\mathbf{Q} \mathbf{Q}^{T}\right)\right] \mathbf{u}^{n+1} } & =\mathbf{f}-\mathbf{Q} \mathbf{p}^{n+1}
\end{aligned}
$$

The incompressibility is imposed only in an average sense [6], that is,

$$
\int_{\Omega} \dot{\varepsilon}_{i i} d \Omega=0 .
$$

Literature by Thompson, et al. [9] and Y. S. Lee, et al., [10] provide information about the free surface adjustment and bulge predictions for a steady state rolling process.

2.4 Heat transfer formulation

Energy balance to solve the heat transfer inside the control volume (see FIGURE 2.2) within a pass is given by,

$$
\left(k \theta_{, i}\right)_{, i}-u_{i} \rho C_{p} \theta_{, i}+\dot{Q}=0 .
$$

In equation (2.30), the first term is the conductive term, second term is the convective term due to the transport of the fluid, and the third term describes the heat generation due to plastic work. The boundary conditions for the thermal problem are,

$$
\text { (a) } \theta=\bar{\theta} \text { on } \Gamma_{\theta}
$$

and

$$
\text { (b) } k \theta_{, i} n_{i}=\bar{q} \quad \text { on } \quad \Gamma_{q}
$$

There is no variational formulation available for energy balance due to the convective terms and hence an application of direct Galerkin's weighted residual method leads to,

$$
[\mathbf{H}]\{\boldsymbol{\theta}\}=\{\mathbf{q}\}
$$


where,

$$
\begin{aligned}
& {[\mathbf{H}]=\underset{1}{\mathbf{A}}\left\{\int_{\Omega^{e}}\left[\mathbf{B}^{\theta}\right]^{T} k(\theta)\left[\mathbf{B}^{\theta}\right] d \Omega^{e}+\int_{\Omega^{e}}\left[\mathbf{N}^{\theta}\right]^{T} \rho C_{p}\{\mathbf{u}\}^{T}\left[\mathbf{B}^{\theta}\right] d \Omega^{e}\right\}} \\
& \{\mathbf{q}\}=\underset{1}{\mathbf{A} e l}\left\{\int_{\Omega^{e}}\left[\mathbf{N}^{\theta}\right]^{T} \dot{\mathbf{Q}} d \Omega^{e}+\int_{\Gamma_{r}^{e}} \kappa \epsilon\left(\theta_{e}^{4}-\theta_{s}^{4}\right)\left[\mathbf{N}^{\theta}\right] d \Gamma^{e}+\int_{\Gamma_{e}^{e}} h_{e}\left(\theta_{e}-\theta_{s}\right)\left[\mathbf{N}^{\theta}\right] d \Gamma^{e}\right\} .
\end{aligned}
$$

The boundary conditions for both flow and heat transfer problem are schematically depicted in FIGURE 2.3 .

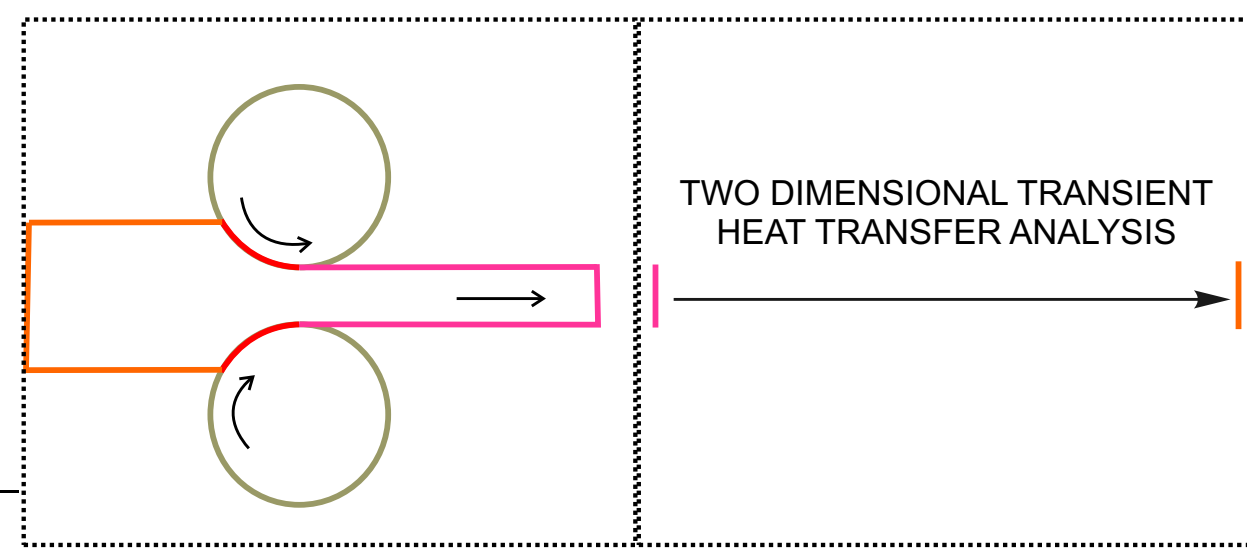

Stand $-i$

Interstand - $i$

Stand - $i$

FIGURE 2.2: 3D and 2D analysis control volumes.

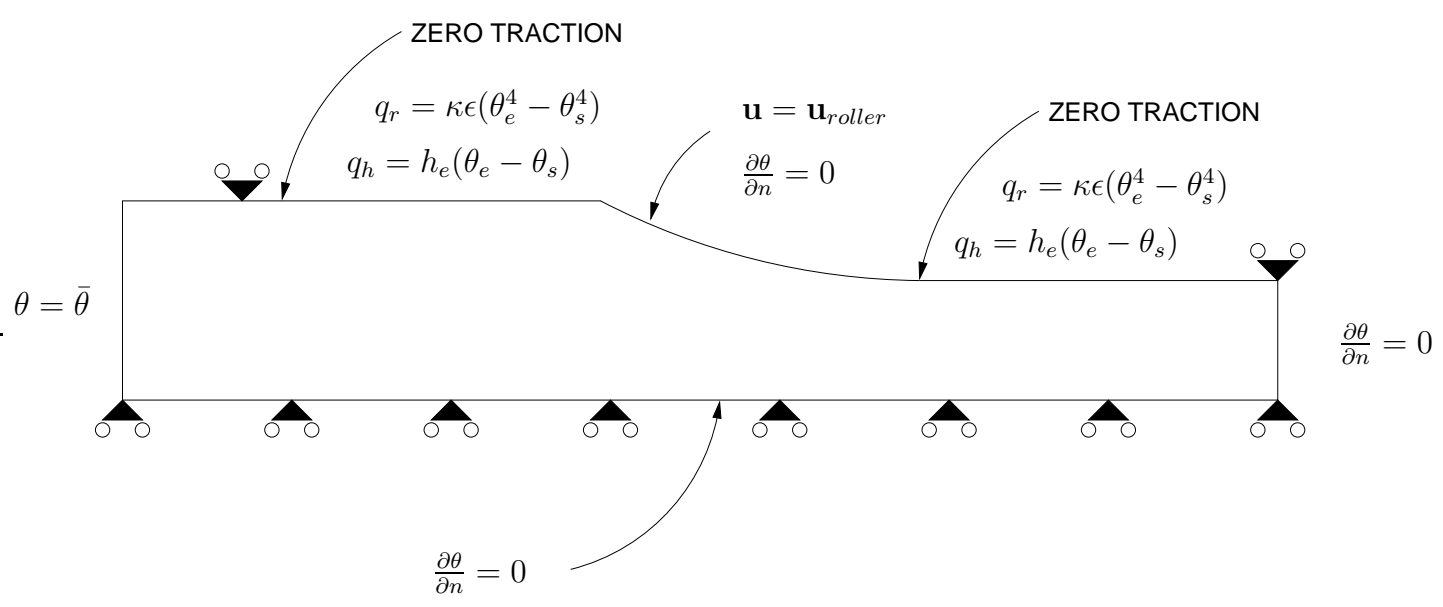

FIGURE 2.3: Boundary conditions used for the 3D analysis in the control volume. 


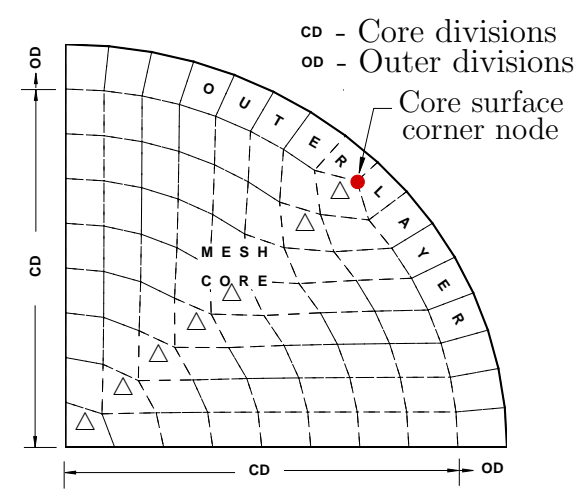

(a) 2D-Mesh.

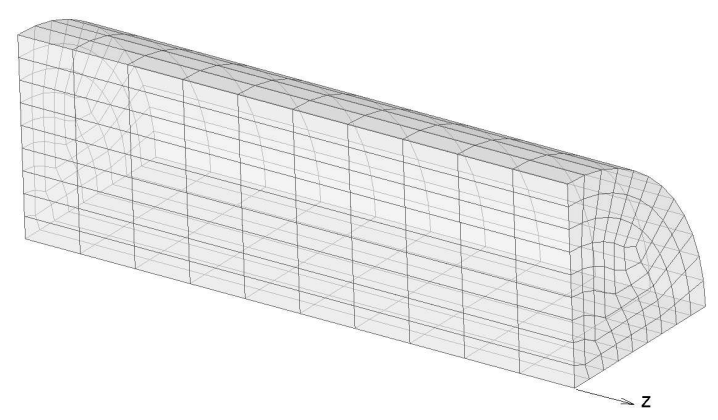

(b) 3D-Mesh.

FIGURE 2.4: Creation of FE mesh used in the multi-pass rolling simulation.

\subsection{Mesh generation}

The FE mesh generation can be summarized as follows. The control volume considered in the analysis is divided into a number (user-specified) of equi-sized slices. Initially, a two-dimensional mesh using quadrilaterals (see FIGURE 2.4(a)) is generated on each cross-section with all the cross-sections having exactly the same nodal connectivity. By specifying the appropriate connectivity between the nodes of crosssectional elements in any given slice, the three-dimensional mesh is created (see FIGURE 2.4(b)). From FIGURE 2.4(a), it can be observed that the two-dimensional mesh is developed as a combination of a core consisting several layers and one or more outer layers. The number of the divisions in the core and outer layers is userspecified.

\subsection{RAWHIDE Capabilities}

As detailed in [1], the above mentioned formulation has been successfully used for a four stand multi-pass rolling. Some of the output variables from RAWHIDE include temperature, effective strain from current stand, effective strain accumulated over the previous stands, effective strain rate, axial and effective stresses, resident time 


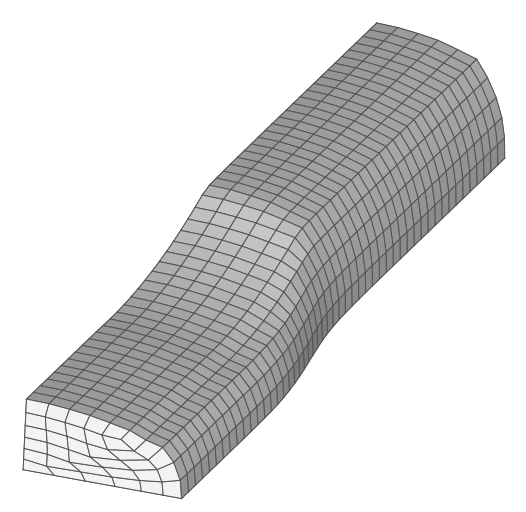

(a) ROLLED BAR AT THE END OF STAND 3

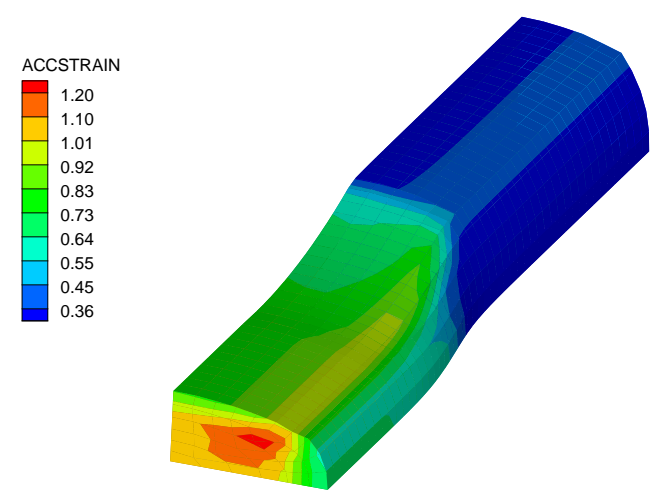

(c) ACCUMULATED STRAIN

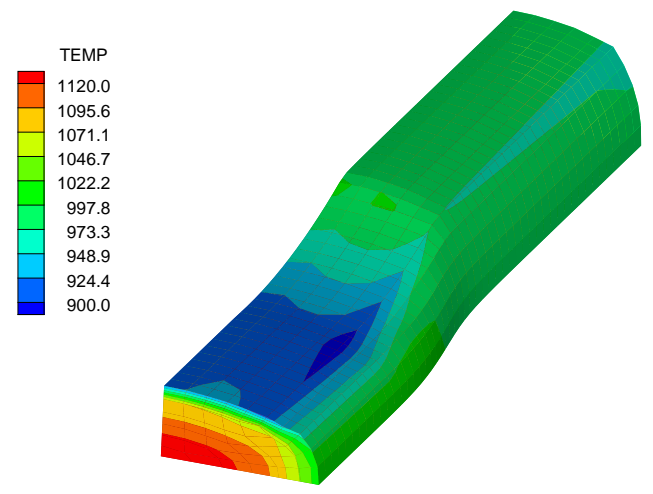

(b) TEMPERATURE DISTRIBUTION

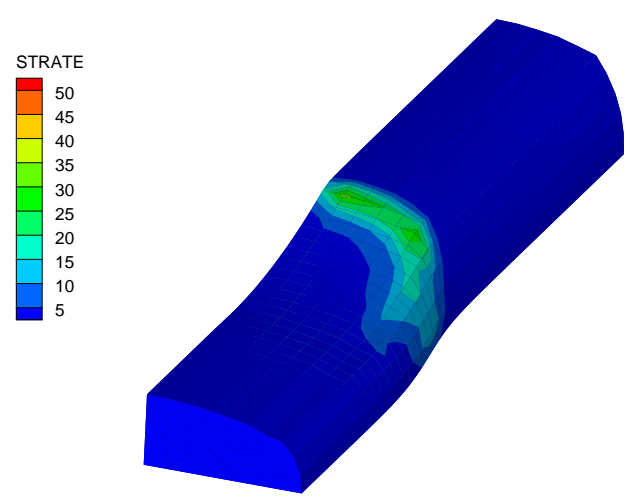

(d) STRAIN RATE DISTRIBUTION

FIGURE 2.5: Steady state control volume after visco-plastic analysis and results from FEM.

and pressure. The important variables that are necessary for a successful prediction of microstructure are the temperature, effective strain from current stand, and the resident time. The resident time is the time that elapses between a material particle's entry into the control volume and the time at its current location in space [11]. Since the analysis is a flow formulated analysis and a material particle is assumed to follow a streamline, this time can be considered for calculating the current strain rate instead of the strain calculated by the analysis for microstructure prediction. 


\section{CHAPTER 3: MESH RE-ZONING IN MULTI-STAND ROLLING}

\subsection{Introduction}

RAWHIDE, the finite-element (FE) package discussed in detail in Chapter 2 is numerically efficient and fast for analyzing the thermomechanical histories during multistand rolling of metals. The package can be used in designing rolling schedules and analyzing process anomalies. The accuracy of the algorithm was demonstrated in [1] by comparing numerical predictions with the experimental results for 4-stand rolling of nickel-base superalloy (alloy 718). However, a typical multistand rolling process consists of as many as 26 stands and as the workpiece passes through these stands, the material deforms significantly (see FIGURE 3.11) and consequently, FE mesh distortion becomes progressively severe. The algorithm presented in [1] does not have remeshing capabilities. In this Chapter, a remeshing technique is presented to handle severe distortions during the rolling process. The technique does not alter significantly the efficiency of the original algorithm.

In addition, even though RAWHIDE carries out a two-dimensional transient heat transfer analysis during the interpass, the temperature history is not recorded during this analysis. It is necessary to have this information in order to predict the microstructure evolution during the interpasses. The major contributors to the microstructure evolution (see Chapter 4 for detailed discussion) are the metadynamic recrystallization (MDRX) and grain growth processes that occur after deformation and further cooling or annealing. Therefore, RAWHIDE package needed a modification to write the temperature histories to accurately predict the microstructure evolution.

\subsection{Mesh distortion}

As detailed in [1], the above mentioned formulation has been successfully used for multi-stand rolling. However, without the re-meshing capabilities, this FE formulation fails at later stands due to severe mesh distortion. A simple angle check revealed 


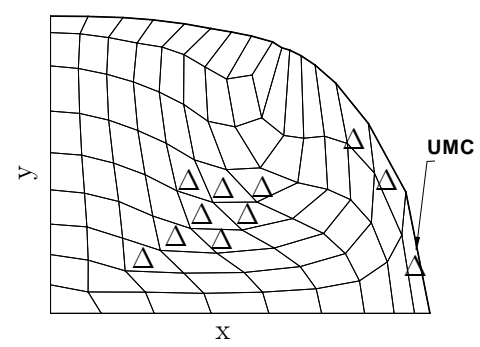

(a) First cross-section

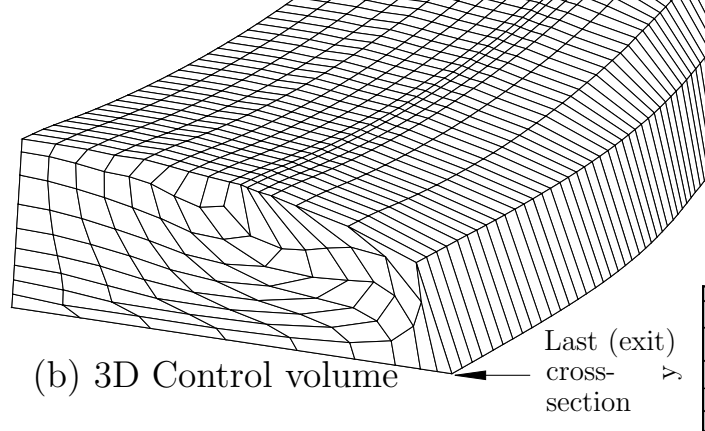

$\Delta \overrightarrow{0}$ - Some of the distorted elements
UMC - Unrealistic missing of surface curvature

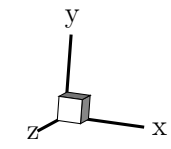
AT THE END OF ODD STANDS
THE SHAPE BECOMES ELLIPTICAL FROM ROUND

First (entrance) cross-

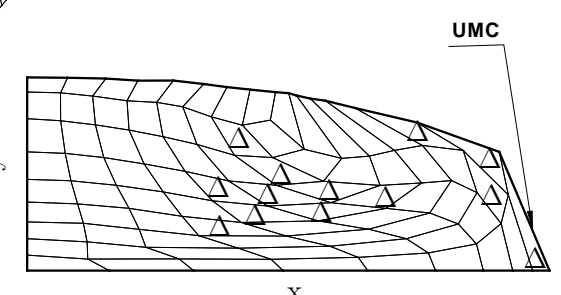

(c) Last cross-section

FIGURE 3.1: Distorted mesh during multi-stand rolling of Alloy 718. The mesh corresponds to $13^{\text {th }}$ stand.

that in many elements in the deformed FE mesh, the included angles were greater than $170^{\circ}$. In this study, an element is defined as distorted when an internal angle exceeds $170^{\circ}$. FIGURE 3.1 shows a case at the end of analysis in the $13^{\text {th }}$ stand. The cross-sections shown are the first (entrance to stand) cross-section (FIGURE 3.1(a)) and the last (exit from stand) cross-section (FIGURE 3.1(c)) of the control volume considered in the analysis. The distorted elements (as defined previously) are marked in these figures with the symbol ' $\Delta$ '. As the analysis progresses over stands, starting from the first stand, near the cross-sections that have gone through the roll-gap, there is an unrealistic loss of bar surface curvature. This is due to the fact that linear elements are used in the analysis and these elements distort significantly as the material passes through the later stands (see FIGURE 3.1(b, c)). Therefore, to proceed further and avoid mesh distortion issues, a remeshing and data interpolation algorithms 
are needed. The next Section provides a discussion of the methodology proposed for remeshing and interpolation collectively called as "mesh rezoning."

\subsection{Outline of the mesh re-zoning approach}

The remeshing algorithm proposed in this work consists of three steps:

1. Fit the outer surface with an algebraic curve.

2. Re-define the mesh by keeping the same number of elements.

3. Interpolate the data from previous mesh to new mesh using an accurate and efficient algorithm.

The first step is needed to restore the bar curvature. The area change after this redefinition of outer surface is assumed to be acceptably small. Since the boundary after this redefinition has an algebraic form, the nodes in the new mesh can be easily found. In order to retain the efficiency and simplicity of the original solution algorithm, the number of nodes (and nodal connectivity) must remain the same before and after remeshing. Step 2 ensures that this requirement is satisfied. Step 3 ensures that the data transfer from old to new mesh does not lead to significant errors or computational cost. The remeshing is essentially addressed as a two-dimensional problem, even though the analysis is three-dimensional. As shown in FIGURE 3.2(a), the cross sectional mesh at the end of the analysis is taken and the boundary defining the mesh is considered as depicted in FIGURE 3.2(b).

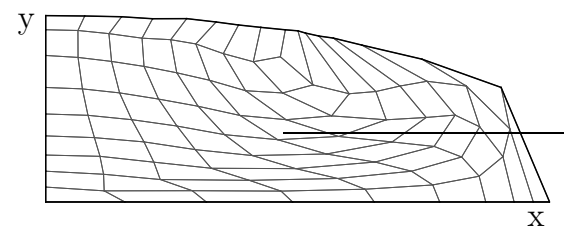

(a) Cross Sectional Mesh

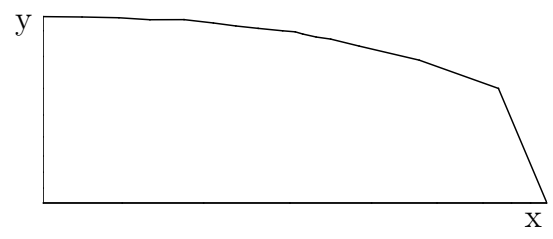

(b) Cross Section Boundary

FIGURE 3.2: Boundary extraction from the FE mesh. 


\subsection{Cubic splines}

Polynomial fits on the outer surface of the distorted mesh may lead to erroneous results because of round-off errors and overshoot. In addition, polynomial fits tend to swing through wild oscillations in the vicinity of an abrupt change in slope on the outer surface which happens around stand 13, (see FIGURE 3.1 (c)) for the data considered in this work. On the other hand, the algebraic curves known as cubic splines are limited to third-order changes, and the oscillations are kept to a minimum and provide superior approximation of the behavior of functions that have local, abrupt changes. Therefore, cubic splines are considered as the appropriate choice of algebraic curves, for the intervals between each pair of contiguous nodes (knots) on the outer surface. Spline functions are lower-order polynomials that are applied to subsets of data points and are widely used in computer graphics. However, the spline-fitting is difficult to automate for the commonly used boundary conditions. 'Natural splines'

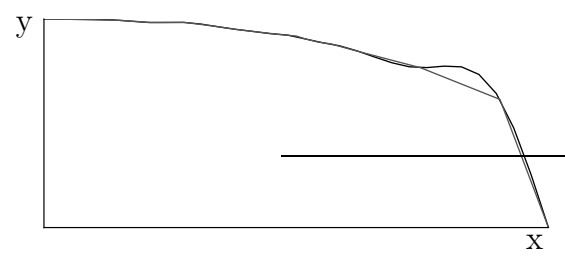

(a) NOT-A-KNOT Spline

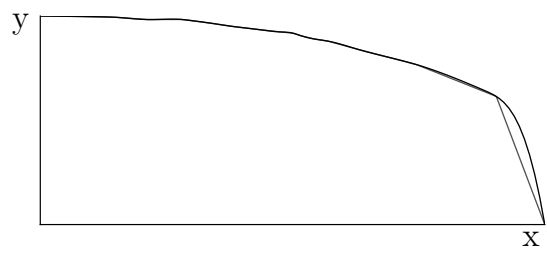

(b) TAUT Spline

FIGURE 3.3: Various spline fits for the mesh boundary.

can be created automatically, but they replicate the original boundary shown in FIGURE 3.2(b) since they approximate the end intervals with a straight line. 'Not-aknot' boundary condition provides smooth fit and can be automated but the problem of 'extraneous-inflection points' (see FIGURE 3.3(a)) needs special treatment with an additional tension parameter as suggested by Schweikert [17]. Algorithms by Cline [18], are available provided the tension parameters are known. Fletcher and McAllister [20] proposed an algorithm for automatic tension adjustment. However, one of the disadvantages is the convergence issues associated with the adjustment of the tension 
parameter. Therefore, their approach becomes semi-automatic and is not preferred in this work. Sapidis, et al., [19] give a solution to find the tension parameters, with a detailed and more involved mathematics. De Boor's [16] simplistic and elegant 'tautsplines' require that the independent data points need to be montonously increasing for convexity preserving splines. The current work involves the independent data points ( $x$-coordinates) that increase monotonically and therefore, become a candidate for taut-spline approximation. The biggest advantage of de Boor's algorithm is the complete automation involved. Due to this reason, de Boor's algorithm is employed here to redefine the outer surface. FIGURE 3.3(b) represents such a fit for the cross section considered.

\subsection{Re-meshing}

As discussed in the beginning of Section 3.3, the number of elements and the nodal connectivity are preserved while re-meshing. As shown in FIGURE 3.1, the largest included angle occurs mainly in the so-called 'diagonal' elements (represented by ' $\triangle$ ' in FIGURE 2.4(a)). The approach considered in this work for remeshing limits this included angle to less than $150^{\circ}$ (see FIGURE 3.4(a)). This is achieved by finding a parametric relation between the coordinates. Then, using a simple geometric approach the core elements can be found based on the core trapezoid (see FIGURE 3.4(b)). The outer layer nodes are the intersecting points between the piecewise cubic taut-spline and a line from the origin passing through the outermost core surface nodes (see FIGURE 3.5(a)). This can be done easily using Newton's method as follows. The piecewise cubic spline on a particular interval is given by,

$$
f(x)_{p p}=a+b\left(x-x_{i}\right)+\operatorname{cfrac}\left(x-x_{i}\right)^{2} 2+d \frac{\left(x-x_{i}\right)^{3}}{6},
$$

where $a, b, c$, and $d$ are the coefficients found from spline-fit applied on the outer surface, and $x_{i}$ are the 'knots' of known coordinates on the outer surface. The straight line with slope $m$ from the origin is,

$$
f_{s}(x)=m x \text {. }
$$




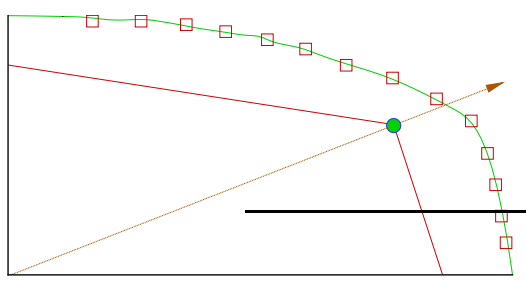

(a) Parametric Core.

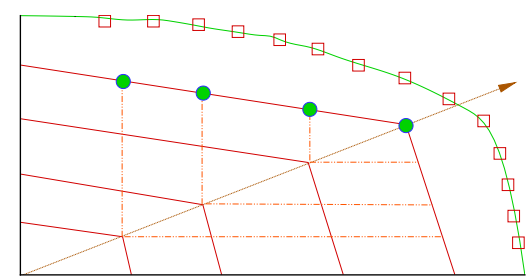

(b) Redefined Core.

FIGURE 3.4: Creation of FE mesh used in the multi-pass rolling simulation (inner layers).

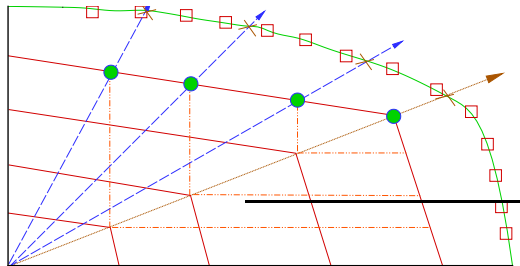

(a) Finding Outer Layer Elements.

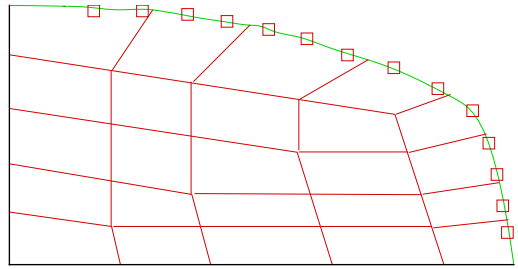

(b) Final Mesh Schematic.

FIGURE 3.5: Creation of FE mesh used in the multi-pass rolling simulation (outer layer).

At the intersecting point,

$$
f_{p p}(x)=f_{s}(x)
$$

and if,

$$
\begin{aligned}
g(x) & =f_{p p}(x)-f_{s}(x), \\
& =a+\left(x-x_{i}\right) b+\frac{\left(x-x_{i}\right)^{2}}{2} c+\frac{\left(x-x_{i}\right)^{3}}{6} d-m x .
\end{aligned}
$$


The solution to the equation $g(x)=0$ is the intersection point and that can be found using Newton's method as per the following expression:

$$
x_{n+1}=x_{n}-\frac{g\left(x_{n}\right)}{g^{\prime}\left(x_{n}\right)} .
$$

The method fails if $g^{\prime}\left(x_{n}\right)$ approaches zero. Evaluating the following terms in equation $(3.4)$,

$$
\left(x-x_{i}\right)^{2}=x^{2}-2 x x_{i}+x_{i}
$$

and

$$
\left(x-x_{i}\right)^{3}=x^{3}-3 x^{2} x_{i}+3 x_{i}^{2} x-x_{i}^{3} .
$$

The derivatives of the above terms can be expressed as,

$$
\frac{d}{d x}\left(x-x_{i}\right)^{2}=2\left(x-x_{i}\right)
$$

and

$$
\begin{aligned}
\frac{d}{d x}\left(x-x_{i}\right)^{3} & =3 x^{2}-6 x x_{i}+3 x_{i} \\
& =3\left(x^{2}-2 x x_{i}+x_{i}^{2}\right) \\
& =3\left(x-x_{i}\right)^{2} .
\end{aligned}
$$

Using equations (3.1), (3.2), (3.4), and from the derivatives determined above, the equation (3.4) can be expressed as,

$$
\begin{aligned}
g^{\prime}(x) & =b+\left(x-x_{i}\right) c+\frac{\left(x-x_{i}\right)^{2}}{2} d-m \\
& =(b-m)+\left(x-x_{i}\right) c+\frac{\left(x-x_{i}\right)^{2}}{2} d .
\end{aligned}
$$

Fortunately, Newton's method needs no modification and works stably due to the first term in equation (3.6). The remeshing becomes a simple geometry problem and can be handled easily. The schematic of a finished mesh would look similar to the one shown in FIGURE 3.5(b). This approach when applied to the cross sections at 
the end of $12^{\text {th }}$ and $13^{\text {th }}$ as benchmarking cases looked as shown in FIGURE 3.6. A more elegant way would be to use the point where the slope changes abruptly on the spline to find the trapezoidal corner node (see FIGURE 2.4(a)) in the core region. This slope change point approach can avoid the larger elements in the outer layer seen in FIGURE 3.6(b), and can be studied in the future for further modification of the remeshing algorithm.

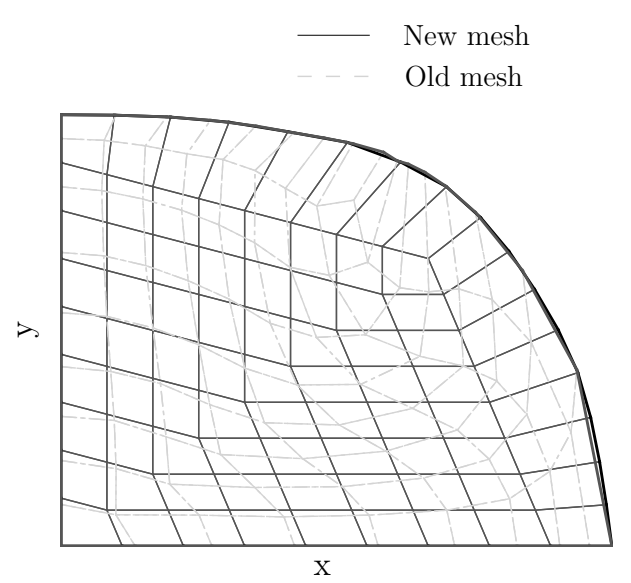

(a) Stand 12 end cross-section
POINT AT WHICH SLOPE CHANGES SHARPLY
CAN BE USED TO FIND THE CORNER POINTS
IN THE CORE FOR THE NEW MESH TO AVOID

THE BIGGER SIZED ELEMENTS AT THE ENDS.

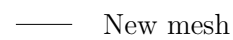

Old mesh

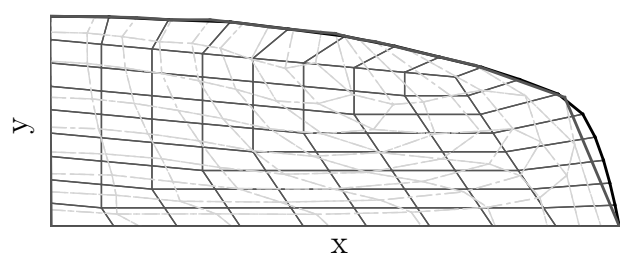

(b) Stand 13 end cross-section

FIGURE 3.6: Redefined mesh during multi-stand rolling of alloy 718.

\subsubsection{Influence on area}

From the benchmarking cases shown in FIGURE 3.6, it can be observed that there is an additional area than the one calculated by the FE simulation. The calculated area increase is found to be $0.267 \%$ for the case shown in FIGURE 3.6 (a) while it is higher at $1.08 \%$ for the case shown in FIGURE 3.6(b). Therefore, the increase is insignificant in the case of round cross sections than the oval cross sections. A simple experiment involving an automatic enforcement of remeshing at every fourth stand was carried out and the results are shown in Table 3.1. From the table, it is clear that the enforcement of remeshing at every fourth stand yields insignificant area increase when compared to the results from an angle based enforcement of remeshing. This is an important feature of the remeshing algorithm since the material is assumed to be 


\begin{tabular}{cc}
\hline Stand No. & \% Increase in Area \\
\hline Stand - & 0.0064 \\
Stand - 8 & 0.0 \\
Stand - 12 & 0.0 \\
Stand - 16 & 0.0 \\
\hline
\end{tabular}

TABLE 3.1: Area increase due to remeshing.

incompressible.

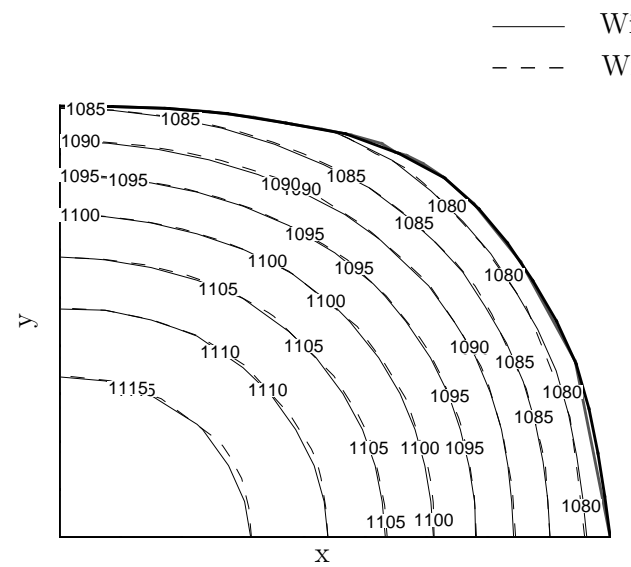

(a) Temperature
With re-meshing \& interpolation

Withoug re-meshing

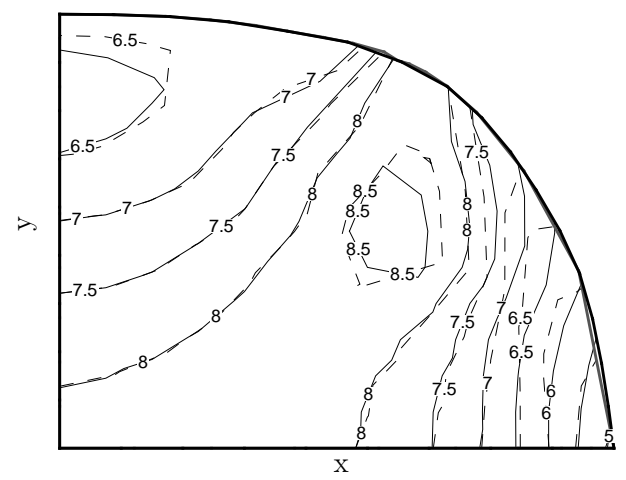

(b) Accumulated Strain

FIGURE 3.7: Re-meshed \& interpolated contour comparison at the end of $12^{\text {th }}$ stand.

\subsection{Interpolation}

The mesh distortion problem associated with the FE models of forming processes were addressed by various authors $[21 ; 22 ; 23 ; 24]$, and accordingly there are various models for re-meshing and interpolating the essential data. The essential data are the history-dependent variables namely temperature, strain, and pressure (needed as starting value for pressure iteration in the next stand). The approach by Crawford, et al., [24] was found to be very simple and easy to automate. Their approach is based on inverting the geometric shape functions of elements of distorted mesh to determine the parameter values of nodes on the new mesh with respect to the old 
mesh [24]. In other words, it is a 'parametric inversion' and is very well suited for a finite element mesh having iso-parametric elements. The parameters involved are essentially the local co-ordinates $\xi$, and $\eta$, for a two-dimensional finite element. If the parameters $\xi$, and $\eta$ are found, then the data transfer is straightforward. In this work, a two-dimensional simplification is considered, that is, data will be transferred to nodes within each cross-section one after the other analogous to the approach used in creating the FE mesh discussed in Section 3.2. This simplification avoids solving an 8-degree polynomial even for a simple tri-linear brick element. In addition, the mesh distortion in the steady-state rolling occurs primarily in the cross-sections, as if the elements are bi-linear quadrilaterals and distortion present in the $z$ direction is negligible. The approach considered involves the following algebra. For an isoparametric element, the geometry and variables are governed by,

$$
x=\sum_{i=1}^{4} N_{i}(\xi, \eta) x_{i}, \quad y=\sum_{i=1}^{4} N_{i}(\xi, \eta) y_{i}, \quad \text { and } \quad u=\sum_{i=1}^{4} N_{i}(\xi, \eta) u_{i}
$$

$N_{i}(\xi, \eta)$ is the bi-linear shape function defined as follows, and $u$ is any variable/data that need to be transferred to the new mesh.

$$
\begin{aligned}
N_{1}(\xi, \eta) & =\frac{1}{4}(1-\xi)(1-\eta), \\
N_{2}(\xi, \eta) & =\frac{1}{4}(1+\xi)(1-\eta), \\
N_{3}(\xi, \eta) & =\frac{1}{4}(1+\xi)(1+\eta), \text { and } \\
N_{4}(\xi, \eta) & =\frac{1}{4}(1-\xi)(1+\eta) .
\end{aligned}
$$

In the above, for a new mesh, the coordinates $x$ and $y$ are known at each new node, and if the bounding old element for any new node is found, all four values of $x_{i}$ and $y_{i}$ are also known and there are two equations in two unknowns ( $\xi$ and $\eta$ ), that can 
be found by applying the process of elimination as follows.

$$
\begin{aligned}
x= & \frac{1}{4}(1-\xi)(1-\eta) x_{1}+\frac{1}{4}(1+\xi)(1-\eta) x_{2}+\frac{1}{4}(1+\xi)(1+\eta) x_{3} \\
& +\frac{1}{4}(1-\xi)(1+\eta) x_{4} \\
= & \frac{1}{4}\left\{[1+\xi \eta-\eta-\xi] x_{1}+[1-\xi \eta-\eta+\xi] x_{2}+[1+\xi \eta+\eta+\xi] x_{3}\right. \\
& \left.+[1-\xi \eta+\eta-\xi] x_{4}\right\} \\
= & \frac{1}{4}\left\{\left[x_{1}+x_{2}+x_{3}+x_{4}\right]+\left[-x_{1}+x_{2}+x_{3}-x_{4}\right] \xi+\left[(\xi-1) x_{1}-(\xi+1) x_{2}\right.\right. \\
& \left.\left.+(\xi+1) x_{3}+(1-\xi) x_{4}\right] \eta\right\} .
\end{aligned}
$$

This can be written as,

$$
x=a_{1}(\xi)+a_{2}(\xi) \eta
$$

where,

$$
a_{1}(\xi)=\frac{1}{4}\left\{\left(x_{2}-x_{1}+x_{3}-x_{4}\right) \xi+\left(x_{1}+x_{2}+x_{3}+x_{4}\right)\right\}
$$

and

$$
a_{2}(\xi)=\frac{1}{4}\left\{(\xi-1) x_{1}-(\xi+1) x_{2}+(\xi+1) x_{3}+(1-\xi) x_{4}\right\}
$$

From equation (3.7), using a similar approach we find that,

$$
y=b_{1}(\xi)+b_{2}(\xi) \eta
$$

where,

$$
b_{1}(\xi)=\frac{1}{4}\left\{\left(y_{2}-y_{1}+y_{3}-y_{4}\right) \xi+\left(y_{1}+y_{2}+y_{3}+y_{4}\right)\right\}
$$

and

$$
b_{2}(\xi)=\frac{1}{4}\left\{(\xi-1) y_{1}-(\xi+1) y_{2}+(\xi+1) y_{3}+(1-\xi) y_{4}\right\}
$$

$a_{1}, a_{2}, b_{1}$, and $b_{2}$ are functions of $\xi$ only and can be found by substituting the bilinear shape functions in equation (3.7) for $x$ and $y$. Now, upon eliminating $\eta$ from 
the equation (3.8) and the equation (3.11),

$$
\begin{aligned}
\eta & =\frac{x-a_{1}(\xi)}{a_{2}(\xi)} \\
& =\frac{y-b_{1}(\xi)}{b_{2}(\xi)}
\end{aligned}
$$

From this equality, $y$ is found as,

$$
y=b_{1}(\xi)+\left[\frac{x-a_{1}(\xi)}{a_{2}(\xi)}\right] b_{2}(\xi)
$$

and

$$
a_{2}(\xi) y=b_{1}(\xi) a_{2}(\xi)+\left[x-a_{1}(\xi)\right] b_{2}(\xi) .
$$

Consequently,

$$
\begin{aligned}
f(\xi) & =a_{2}(\xi)\left[y-b_{1}(\xi)\right]+\left[a_{1}(\xi)-x\right] b_{2}(\xi) \\
& =y a_{2}(\xi)-a_{2}(\xi) b(\xi)+a_{1}(\xi) b_{2}(\xi)-x b_{2}(\xi) \\
& =\left[a_{1}(\xi) b_{2}(\xi)-a_{2}(\xi) b_{1}(\xi)\right]+\left[y a_{2}(\xi)-x b_{2}(\xi)\right] .
\end{aligned}
$$

The $\xi$ that satisfies the equation $f(\xi)=0$, can be found iteratively using the Newton's method as follows,

$$
\xi_{n+1}=\xi_{n}-\frac{f(\xi)}{f^{\prime}(\xi)} .
$$

Assume,

$$
\begin{aligned}
& c_{1}(\xi)=a_{1}(\xi) b_{2}^{\prime}(\xi)+a_{1}^{\prime}(\xi) b_{2}(\xi), \\
& c_{2}(\xi)=a_{2}(\xi) b_{1}^{\prime}(\xi)+a_{2}^{\prime}(\xi) b_{1}(\xi), \\
& d_{1}(\xi)=a_{2}^{\prime}(\xi),
\end{aligned}
$$

and

$$
d_{2}(\xi)=b_{2}^{\prime}(\xi)
$$


From equations (3.9), (3.10), (3.12), and (3.13),

$$
\begin{aligned}
a_{1}^{\prime}(\xi) & =\frac{1}{4}\left(x_{2}-x_{1}+x_{3}-x_{4}\right) \\
a_{2}^{\prime}(\xi) & =\frac{1}{4}\left(x_{1}-x_{2}+x_{3}-x_{4}\right) \\
b_{1}^{\prime}(\xi) & =\frac{1}{4}\left(y_{2}-y_{1}+y_{3}+y_{4}\right)
\end{aligned}
$$

and

$$
b_{2}^{\prime}(\xi)=\frac{1}{4}\left(y_{1}-y_{2}+y_{3}-y_{4}\right)
$$

By using the above expressions, equation (3.14) can be rewritten as,

$$
\xi_{n+1}=\xi_{n}-\frac{\left[a_{1}\left(\xi_{n}\right) b_{2}\left(\xi_{n}\right)-a_{2}\left(\xi_{n}\right) b_{1}\left(\xi_{n}\right)\right]+\left[y a_{2}\left(\xi_{n}\right)-x b_{2}\left(\xi_{n}\right)\right]}{\left[c_{1}\left(\xi_{n}\right)-c_{2}\left(\xi_{n}\right)+y d_{1}\left(\xi_{n}\right)-x d_{2}\left(\xi_{n}\right)\right]} .
$$

Choose $\xi_{0}=0,1,-1$ and iteratively check $\left|\xi_{n+1}-\xi_{n}\right|<$ Tol, where Tol is the tolerance criterion to find $\xi$. The solution to equation (3.15) that lies in the interval $[-1,+1]$ is the acceptable solution. Degeneracies did occur with this approach, as also noted by Crawford, et al., [24]. These degeneracies were overcome by providing an additional algorithm called 'PNPOLY', developed by W. R. Franklin [25] based on one of Haines's [26] approaches for testing a point inside a polygon. The mathematics behind this is a simple shoot-a-ray method developed using Jordan Curve Theorem in geometry. An immediate application of this algorithm is to check if a new node lies inside any old element. Then the parameter $\xi$ is calculated based on equation (3.15) and checked for their interval validity. Once $\xi$ is determined, $\eta$ can be found in closed-form. However, this approach fails on the boundary because the new boundary falls outside the volume contained by the old mesh. Therefore, for the new boundary nodes one can use the same spline-fit for the data. Linear interpolation/extrapolation is used for the boundary nodes as it is just sufficient for the dataset. Two of the key variables in the analysis, the temperature and strain are compared in FIGURES 3.7 (a) and (b) for the $12^{\text {th }}$ stand before and after re-meshing. The data interpolated are in excellent agreement with each other. 


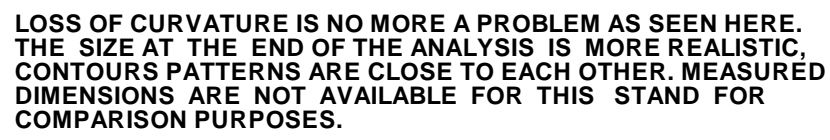

_ Strains for re-meshed geometry

- _ - Strains for geometry without re-meshing

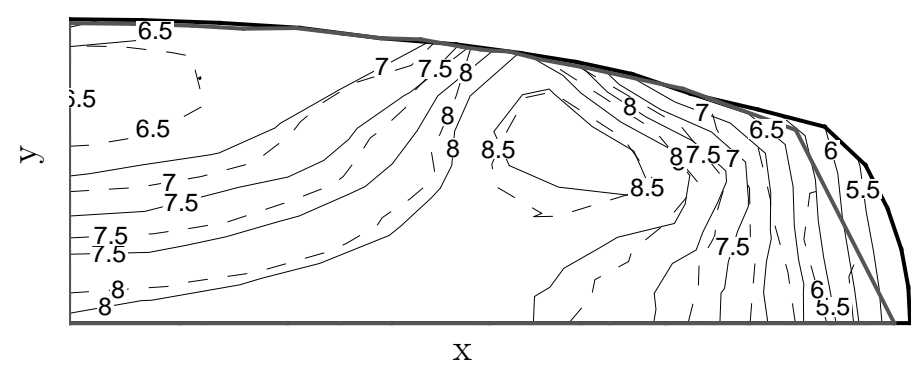

FIGURE 3.8: Strain contours.

\subsection{Results from mesh re-zoning}

The algorithm proposed by Thompson, et al., [1] was tested with the newly incorporated mesh re-zoning algorithms explained in Section 3.3 and found to work very well. The FE algorithm was coded in such a way that mesh re-zoning is user controlled. That is, the user has the options such as an angle based automatic remeshing option and remeshing at every $n^{\text {th }}$ even stand. The data gets updated for that stand, before being read for analysis in the next stand. The example considered here involves hot rolling of a circular bar of alloy 718 (the same material considered by Thompson, et al., [1]) through 16 stands. For a mesh with 5 core divisions and 1 outer divisions the shape prediction is compared in FIGURE 3.9 and the predicted shapes are in good agreement with the factory given samples for the same material and rolling parameters. As another benchmark, for a mesh with 8 core divisions and 1 outer division (FIGURE 2.4(a)) along the width (maximum $x$-coordinate) and height (maximum $x$-coordinate) considered in Section 3.1, mesh re-zoning occured 

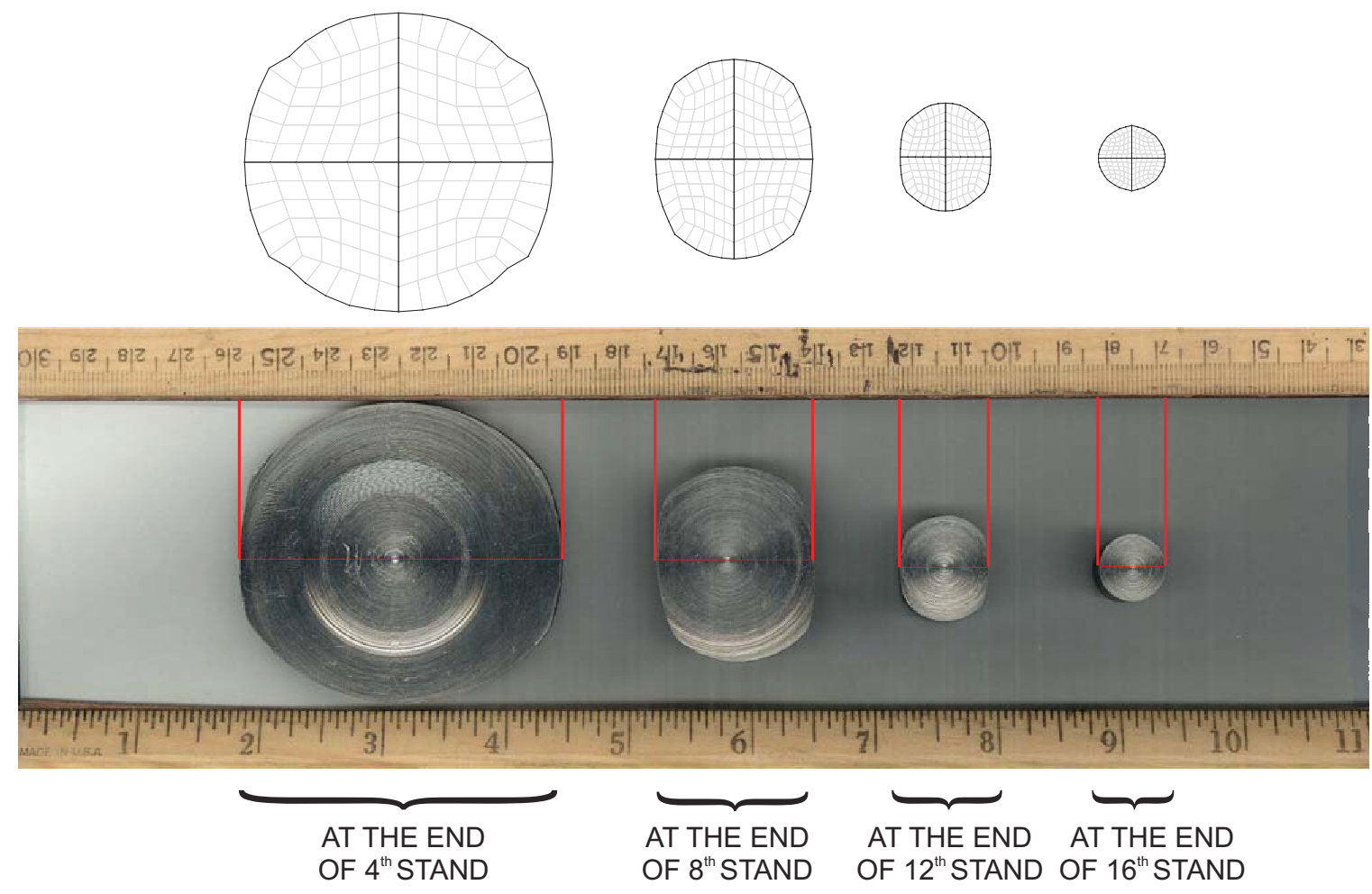

FIGURE 3.9: Comparison of predicted shapes.

at stands 5 and 12. In FIGURE 3.8, the contour levels at the end of the $12^{\text {th }}$ stand are compared for with and without re-zoning. As is evident from this figure, the contours match closely in the interior regions. A comparison with the analysis predicted with remeshing (PWR) dimensions, analysis predicted without remeshing (PWOR) dimensions, and measured (M) dimensions are listed in Table 3.2. It can be observed that the predicted dimensions are in close agreement with the measured dimensions. Further, the predicted dimensions when remeshing is enforced are close to the measured values. There is no data available in the table for predicted dimensions from the analysis carried out without remeshing (that is, PWOR), due to the breakdown of analysis at the $13^{\text {th }}$ stand.

\subsection{Interpass temperature history}

A change in the original RAWHIDE was incorporated to write the history of temperature while carrying out the two-dimensional heat transfer analysis during the interpasses. This is important in predicting the microstructural evolution accurately. A detailed discussion for the necessity of this change is provided in Chapter 4. Prior 


\begin{tabular}{llcc}
\hline & & $\begin{array}{c}\text { Width } \\
(\mathrm{mm})\end{array}$ & $\begin{array}{c}\text { Height } \\
(\mathrm{mm})\end{array}$ \\
\hline Stand-4 & PWR & 63.00 & 65.26 \\
& PWOR & 63.00 & 65.26 \\
& M & 63.06 & 67.31 \\
Stand-8 & PWR & 40.86 & 32.14 \\
& PWOR & 40.75 & 32.10 \\
& M & 40.96 & 32.48 \\
Stand-12 & PWR & 21.90 & 18.32 \\
& PWOR & 21.50 & 18.43 \\
& M & 22.05 & 18.17 \\
Stand-16 & PWR & 14.64 & 13.18 \\
& PWOR & - & - \\
& M & 13.76 & 13.68 \\
\hline
\end{tabular}

TABLE 3.2: Predicted Vs. Measured.

to the incorporation of this modification RAWHIDE plotted temperatures linearly in the interpasses. However, the variation of temperature may not be linear as shown in the FIGURE 3.10(b). FIGURE 3.10(b) illustrates the temperature history of the node at the center of the billet (described by a dot at the origin in FIGURE 3.10(a)). The results were extracted from a 16 stand rolling analysis for the Alloy 718 including a 2 seconds air quenching followed by 2 second water quenching at the end of the $16^{\text {th }}$ stand. The inset in the FIGURE shows the temperature distribution of center node during the first interpass.

\subsection{Conclusion}

FIGURE 3.11 shows the reduction in area during the rolling process for a 16-stand continuous rolling. The reduction is of such magnitude that, the analysis interrupts in the midway due to severe mesh distortion. A systematic approach to address the mesh distortion and associated unrealistic material reduction problems in a continuous multi-stand shape rolling has been developed and validated. The approach enforces both remeshing and data transfer from the old to the new mesh. The data transfer is done in a completely automated fashion without any of the parameter 


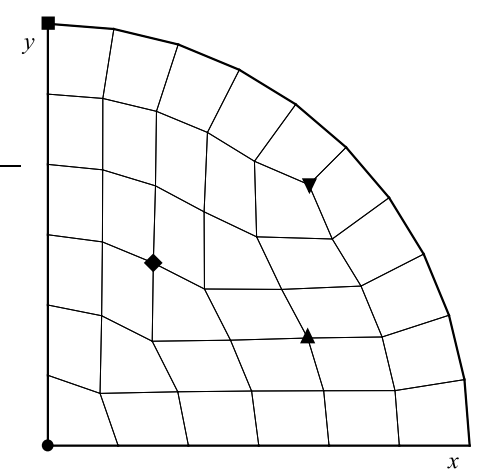

(a) Initial Cross Section.

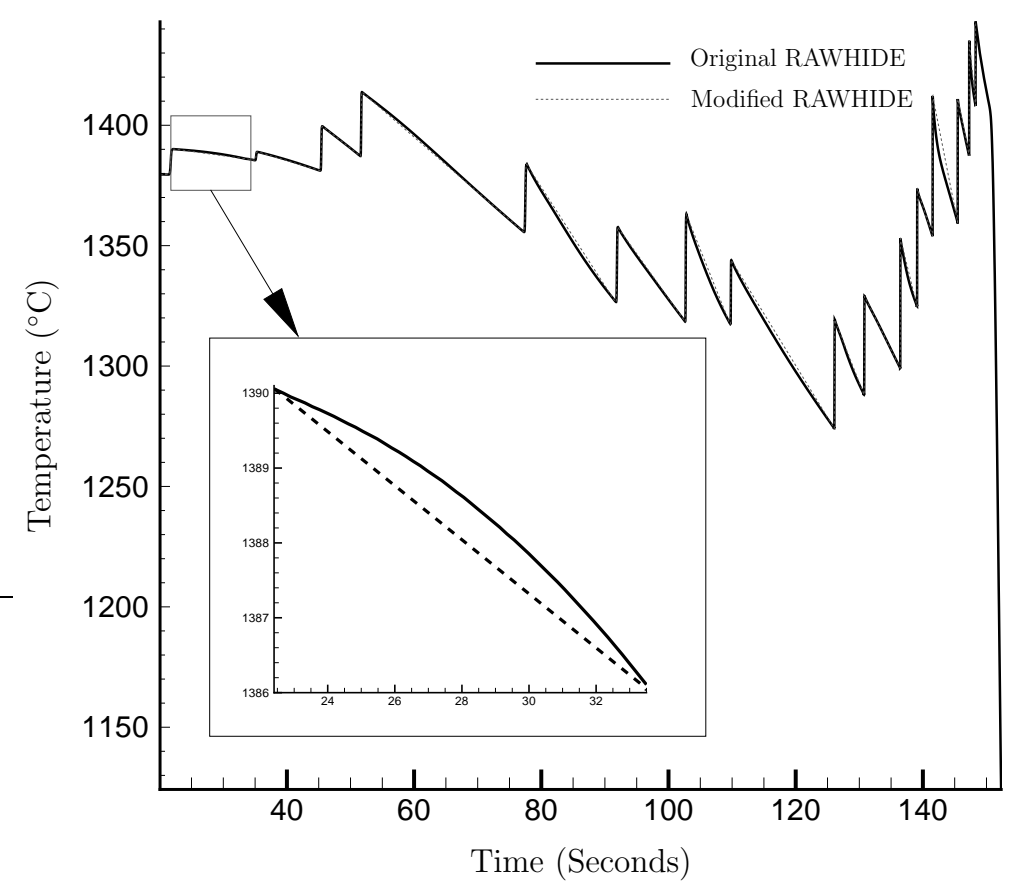

(b) Result for Center Node.

FIGURE 3.10: Temperature history output. 


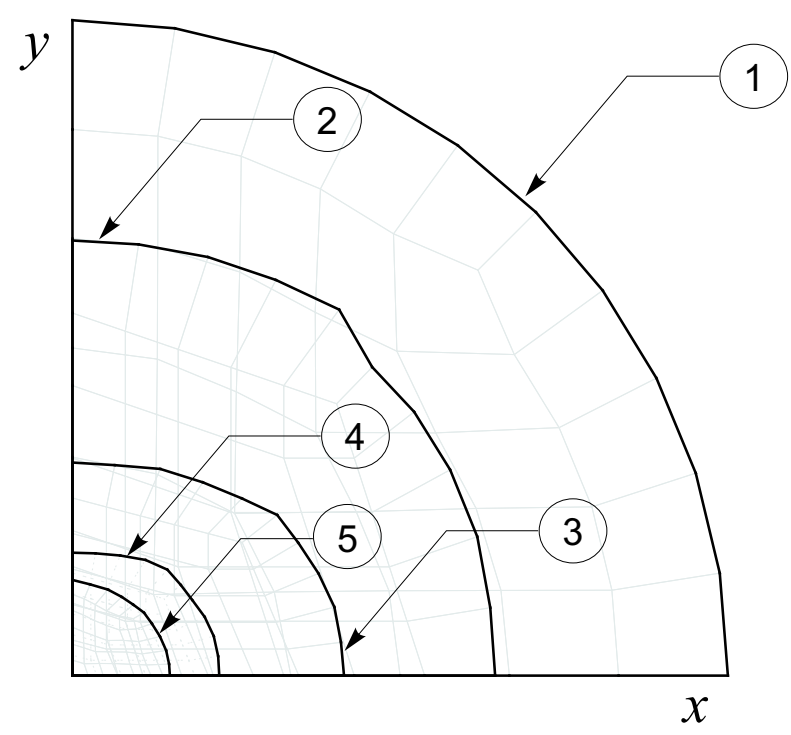

(1) Initial cross section of the billet

2 Cross section at the end of $4^{\text {th }}$ stand ( $56 \%$ Area Reduction)

(3) Cross section at the end of $8^{\text {th }}$ stand ( $~ 86 \%$ Area Reduction)

(4) Cross section at the end of $12^{\text {th }}$ stand ( $95 \%$ Area Reduction)

(5) Cross section at the end of $16^{\text {th }}$ stand ( $98 \%$ Area Reduction)

FIGURE 3.11: Reduction in area during continuous rolling.

degeneracies noted in [24] using a similar approach. An additional modification of including temperature in the history output during interpass analysis was incorporated in the original RAWHIDE package. 


\section{CHAPTER 4: MICROSTRUCTURE THEORY AND RELATED WORK}

\subsection{Introduction}

In the case of Alloy 718, work hardening and dynamic softening coexist during hot deformation [65]. Hardening is mainly due to the increase of dislocation density in the superalloy during the deformation process. Various deformation parameters (such as strain, strain rate, and temperature) influence the microstructure. During deformation, strain causes the increase of dislocation density which results in the work hardening. The strain rate accelerates the accumulation of dislocations that results in the strain-rate hardening. Temperature is related to the softening process through the resulting decrease or rearrangement of dislocations. In general, the thermomechanical processing encompasses recovery, recrystallization and grain growth. Recovery and/or recrystallization may occur during deformation at high temperatures which are the common softening or restoration processes. In addition, the rates of cooling of the material are generally very low in large-scale metal forming operations, allowing recovery, recrystallization and grain growth to occur immediately after hot deformation. These dynamic restoration processes are different from the static annealing processes which occur during post-deformation heat treatment. These processes are of special importance to the metal industry. This is due to the fact that, these processes lower the flow stress of the material. Therefore, these restorative processes enable the material to be deformed more easily. In addition, they also have an influence on the texture and the grain size of the worked material. However, these processes are not well understood since they are difficult to study experimentally and model theoretically.

\subsubsection{Recovery}

It has been observed that recovery lowers the driving force for recrystallization [94]. The kinetics of recrystallization is influenced by a significant amount of prior recovery. Even though, it is very difficult to distinguish recovery and recrystallization, recovery 
mechanisms influence the nucleation in recrystallization. Complete recovery can only occur if the polycrystalline metal is lightly deformed. However, if the crystals are deformed into stages II or III of work hardening [95], then recrystallization intervenes the recovery. That is, highly strained materials undergo recrystallization when compared to recovery. This is attributed to the annealing behavior of the various types of dislocation structure produced during deformation.

\subsubsection{Recrystallization}

Dynamic recovery is dominant in high stacking fault energy (SFE) metals such as aluminum and its alloys, $\alpha$-iron and ferritic steels. This recovery process is rapid and extensive at high temperatures. In the case of metals of low or medium stacking fault energy (copper, nickel and austenitic iron), the recovery processes are slow, and dynamic recrystallization dominates after a critical deformation condition is reached. Dynamic recrystallization has clear nucleation and growth stages and is classified as

a discontinuous process [94]. During dynamic recrystallization, new grains originate at the old grain boundaries. As the material continues to deform, the dislocation density of the new grains increases, thus reducing the driving force for further growth. In addition, the nucleation of further grains at the migrating boundaries limits the growth of the new grains. This leads to a thickening band of recrystallized grains. If there is a large difference between the initial grain size and the recrystallized grain size, then a 'necklace' structure (see FIGURE 4.1) of grains may be formed, and eventually the material will become fully recrystallized.

\subsubsection{Parameters}

The progress of recrystallization during isothermal annealing is commonly represented by a plot of the volume fraction of material recrystallized (X) as a logarithmic function of the independent variable as follows,

$$
X=1-\exp \left(-\beta x^{n}\right)
$$

The independent variable $(x)$ in equation (4.1) can be time or strain, depending on the phase of recrystallization process discussed later. This is commonly known as the 


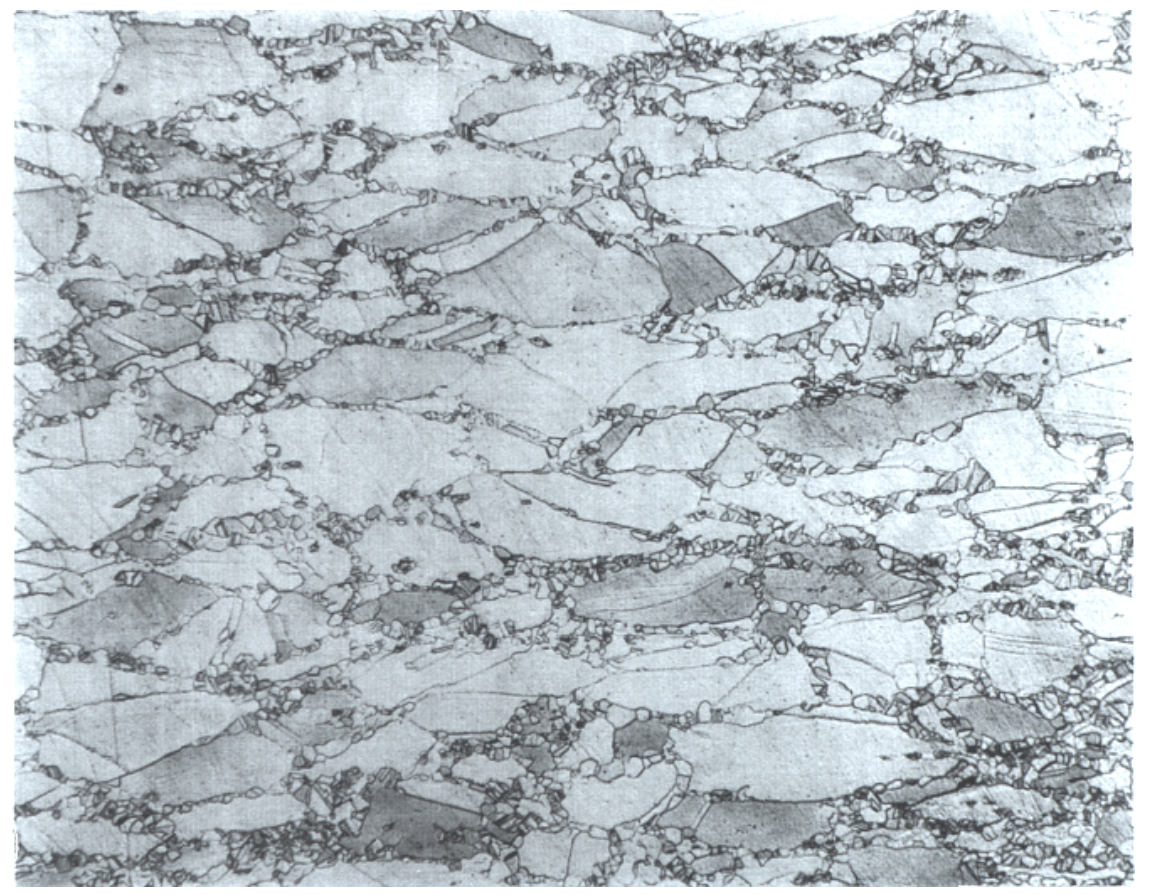

FIGURE 4.1: Microstructure on the surface of an as-rolled billet showing 'necklace' structure (Courtesy: ATI Allvac).

Johnson-Mehl-Avrami-Kolmogorov (JMAK) model [94]. Equation (4.1) is typical of many transformation reactions, and has been described in terms of the constituent nucleation and growth processes. $\beta$ is typically a function of the rate at which the nuclei are formed, and the rate at which the grains grow. The exponent $n$ is usually defined as JMAK or Avrami exponent. The significant feature of the JMAK approach is that the nucleation sites are assumed to be randomly distributed. However, it is too simple to quantitatively model a process as complex as recrystallization. Graphically, equation 4.1 represents a sigmoidal curve (see FIGURE 4.2) showing an apparent incubation time before any recrystallization is detected. Then, an increasing rate of recrystallization is detected by a linear region, ultimately, leading to a decreased recrystallized region. In general, for isothermal experiments, the measure of the rate of recrystallization is characterized by the value of $x$ at which recrystallization is $50 \%$ complete. The microstructural evolution is usually dependent on the deformation temperature $(\mathrm{T})$ and strain rate $(\dot{\varepsilon})$ in addition to the strain $(\varepsilon)$ at temperatures where thermally activated deformation and restoration processes occur. The strain 


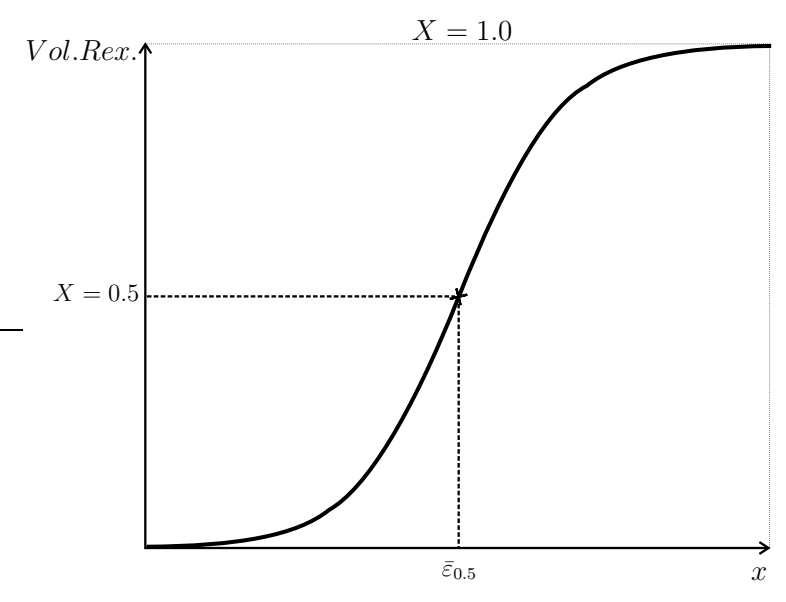

FIGURE 4.2: DRX based on Strain.

rate and deformation temperature are often incorporated into a single parameter, the 'Zener-Hollomon parameter' $(Z)$ also known as the 'temperature compensated strain rate.' This parameter is defined as:

$$
Z=\dot{\varepsilon} \exp \left(\frac{Q}{R T}\right)
$$

where $Q$ is the activation energy of the process. $Z$ is closely related to the flow stress and hence to the dislocation density. The Zener-Hollomon parameter is particularly convenient for discussions of hot working processes in which the temperature and strain rate are generally known, whereas the flow stress may not be measurable. It should be noted that the flow stress is incorporated into RAWHIDE discussed in Chapter 1 as a function of temperature, strain, and strain rate in a tabular form.

\subsection{Microstructural evolution}

From the brief discussion about the restoration processes and the parameters that characterize the microstructure, it is clear that during high strain and strain rate thermomechanical processes such as multi-pass rolling, recrystallization is the major restoration process that influences the development of microstructure. Therefore, further discussion will focus on the details of recrystallization alone. Recrystallization based microstructure evolution may involve the following phases,

- Dynamic recrystallization (DRX) 
- Metadynamic recrystallization (MDRX)

- Static recrystallization (SRX) and recovery/grain growth (SGG)

\subsubsection{DRX}

DRX occurs during deformation when the strain exceeds a certain critical strain $\bar{\varepsilon}_{c}$. This occurs somewhat before the peak of the stress-strain curve. For a range of testing conditions, maximum stress is uniquely related to the Zener-Hollomon parameter (Z). $\varepsilon_{c}$ decreases steadily with decreasing stress or Zener-Hollomon parameter [30]. In this process, the nuclei for recrystallization are formed. DRX is associated with very high dislocation densities and correspondingly high energy levels. It is a very unstable process involving partial recrystallizations and depends on pre-heating, hot-working temperature, imposed strain and strain rate. DRX may also occur during creep deformation [94]. However, the main difference is that the hot working is generally carried out at higher strain rates $\left(1-100 \mathrm{~s}^{-1}\right)$, whereas, strain rates during creep are very low (below $10^{-5} \mathrm{~s}^{-1}$ ).

\subsubsection{MDRX}

MDRX occurs after deformation because the strains required to complete the DRX are not continuously achieved. The strains are still greater than the critical strain $\bar{\varepsilon}_{c}$. During MDRX, no new nuclei are formed but the dislocations density reduces. Even though the straining is stopped, annealing continues and the existing nuclei will grow with no incubation period [30] into the heterogenous, partly dynamically recrystallized matrix. In this heterogenous matrix, the dislocation free nuclei formed during DRX continue to grow during the early stages of post deformation annealing due to MDRX [94]. MDRX is also an unstable process but the grain structure transforms to a fully recrystallized structure. There are other unrecrystallized regime with a high dislocation density in the matrix that also undergo MDRX, however, some literature cited in the Reference [94] classify them as SRX, discussed in the next Section.

\subsubsection{SRX}

Static recrystallization may occur when a hot deformed material is subsequently annealed as mentioned in MDRX. This is very similar to the MDRX, however, stored 
energy is lower than the previously mentioned processes. SRX is also strongly dependent on $Z$ and the effect of strain is also important, and in materials that undergo DRX, the SRX depends on whether the strain was larger or smaller than that required for DRX. The current work does not distinguish MDRX and SRX as separate processes and only MDRX is considered for further analysis.

\subsubsection{SGG}

When the material is fully recrystallized, then further grain growth may occur. Static grain growth occurs after deformation. The strains are less than the critical strain $\bar{\varepsilon}_{c}$. Grain growth is a stable process.

\subsection{Empirical modeling}

Empirical laws describing the various processes mentioned above establish the relationships between microstructural features such as, grain size, texture, and processing parameters such as tool and workpiece geometry, temperature, deformation speed,

and amount of deformation through regression analysis of experimental data [45; 49].

\subsection{Related work}

The previous Sections provided an overview of the microstructural processes that were the subject of study in predicting the microstructure evolution during various thermomechanical processing of different materials. Various authors have chosen different approaches depending on the behavior of any given specific material to evaluate the microstructural evolution. Sizable research has been carried out in predicting the evolution of microstructure during hotworking of superalloys over the last three decades. The following gives a comprehensive survey of the literature related to the microstructural evolution pertinent to the current work.

\subsubsection{Constitutive laws}

One of the original and important publications on the recrystallization and grain growth during hot deformation, can be attributed to Sellars [30]. Sellars considers the relationship between the grain size obtained after each process (DRX, MDRX and SRX) and the stress. This publication discusses the initiation of various processes with reference to a certain critical strain. Further, the work by Sellars emphasized that the deformation is a process taking place by dislocation movements rather than by 
diffusion mechanism theories existing at that time. The evolution of microstructure was studied as a function of temperatures, pass reductions, speeds, and times in rolling schedules by Sellars and Whiteman [28] during the plate rolling of low carbon manganese steel. The constitutive equations were written in terms of the temperature during the deformation, strains (which is a function of dislocation density) and strain rate for the various recrystallization and recovery processes. Smelser, et al. [3], consider a material structure based internal state variable to find the constitutive laws for extrusion process. The use of an internal variable constitutive model has allowed a detailed comparison of the evolution of microhardness in the deformation zone and subsequent product. The use of numerical techniques is one of the key features of this study. Brown, et al. [76], also describe an internal variable constitutive model for hot working of metals. Constitutive equations for cyclic plasticity of waspaloy were proposed by Latif [55] to overcome the flaws encountered in the previous kinematic hardening models. Davenport, et al. [31], describe the flow stress behavior or steel during hot deformation. This work standardizes the constitutive equations in terms of $Z$ which in turn, is a function of temperature and strain rate. Hot torsion tests were conducted by Hodgson, et al. [32], to study the microstructural changes during interpass times and accumulated strains common in rod and bar rolling. In this work, the accumulated strain from pass to pass in C-Mn steel rod rolling was used to calculate complete dynamic recrystallization. Shen $[49 ; 50 ; 51]$, considered the forging of waspaloy turbine discs. Shen developed constitutive equations involving the $Z$ and carefully studies the effect of DRX, MRX, and SRX on the microstructure evolution. The constitutive equations were developed in line with a series of experiments carried out using Gleeble testing machine and realtime forgings at a factory. Kuziak, et al. [47] followed the approach of Gangshu, to predict the microstructure in the forging of nickel-base superalloy bolt heads.

The current work takes into account the empirical laws proposed by Gangshu [49] as the basis. However, the development of the proposed microstructural algorithm incorporates appropriate modifications to predict the behavior of various superalloys including Alloy 718. 


\subsubsection{Computations}

The research works mentioned in the previous Section involved a tremendous amount of time and effort in carrying out experiments and establishing the constitutive models for the various recrystallization processes. However, as mentioned earlier in Chapter 1 industrial trials and laboratory experiments are very restrictive in the information they provide. The advancement in the computer industry, better plastic deformation constitutive laws, and numerical procedures such as Finite Element Methods (FEM) to simulate complicated thermomechanical processing provided the impetus for further development in the prediction of microstructure. The constitutive equations describing the microstructure evolution need parameters such as temperature, strain and strain rates. These can be found using the FEM by commercial packages such as FORGE [47; 73; 75], ABAQUS [38], LARSTRAN/SHAPE [74], DEFORM [43; 64] and RAWHIDE discussed in Chapter 1.

Anderson, et al. [82], were some of the pioneers in numerically simulating the grain growth in materials during the early 80's. The use of FEMs picked up slowly and by the end of the twentieth century, there was substantial work done in modeling of microstructural evolution. The microstructural modeling coupled with the plastic deformation could predict various hotworking processes. Also, this helped in coming up with newer and better constitutive models for different materials. The microstructural phenomena can be studied even more precisely with the advent of the faster computers and quasicontinuum formulations [79].

Beynon \& Sellars developed SLIMMER (sheffield Leicester Integrated Model for Microstructural Evolution in Rolling) [27]. This program can calculate rolling loads and torques using Sims theory with an accurate prediction of mean flow stress. In their model, they consider the dynamic recovery. The predominant microstructural feature of dynamically recovering metals is a well-defined subgrain structure. Modeling the dynamic microstructural events is important for determining flow stress levels, and hence rolling loads. A more detailed work was carried out by Habraken \& Bourdouxhe [15] on steel pieces when the materials were cooling from high temperature. In this work, they use the additivity principle, that is, the microstructure obtained 
at the end of a continuous cooling is a result of a succession of elementary isothermal transformations, each one independent of the preceding thermal history. The model considers germination/nucleation being achieved if the Scheil's sum reaches unity. This is an extensive work involving the total strain rate incorporating five terms: elastic, thermal, transformation, plastic, transformation plastic. A recent publication by Lin, et al. [33] treats the microstructure evolution in the context of dislocation densities using viscoplastic equations for C-Mn steel.

Mirza, et al. [77], incorporated microstructure predicting algorithms in a FE package to determine the microstructure in aluminum alloys. Goerdeler, et al. [74], and Hirch, et al. [78], developed simulation procedures that can predict the grain orientation or texture in addition to the usual grain size prediction during the multi-pass rolling of aluminum alloys. The relative position of the peak in the flow stress shifts as a function of the $Z$ complicating the analyses. Davenport, et al. [31], suggest the incorporation of constitutive equations into first stage equations, describing the stress at a given strain as a function of $Z$, and second stage equations, resulting in a continuous flow stress curve. Serajzadeh [34; 35], in his publications discusses an approach involving the basic balance laws coupled with the microstructural behaviors. The vast number of experiments and numerical procedures carried out for steel materials have been compiled in the form of a textbook [37] listed in the References. This literature incorporates the many procedures developed for microstructural evolution during various processes and the established constitutive laws for various iron alloys.

The current work employs the FEM calculated deformation variables from the custom built FE code, RAWHIDE and uses the formulation discussed in the next Section to model the microstructure as a separate microstructure analysis package.

\subsubsection{Formulations}

Some of the published works take specific recrystallization processes into consideration. The effects of DRX and MRX are discussed by Zhou \& Baker [46] on wrought Alloy 718 in hot deformation and the kinetics of DRX is predicted by Serajzadeh [36]. Semiatin, et al. [48], divide the DRX into discontinuous DRX (DDRX) and continuous DRX (CDRX) for low stacking fault-energy materials such as nickel based alloys. 
Some authors tried to modify the FEM with reference to the basis such as Lagrangian [38], Eulerian and mixed formulation [7] in predicting the microstructure evolution. Josef, et al. [7], develop a velocity-displacement model (or vu-model) under planestrain conditions for hot rolling process which is a better model when compared with the velocity-pressure model commonly found in the commercial FEM packages. Karhausen, et al. [39], provide a comprehensive procedure in implementing the microstructure prediction during a five pass rolling of $\mathrm{Cr}-\mathrm{V}$ Steel. In this procedure, the effective strain used in the calculations is assumed to be a function of the volume recrystallized during the rolling process. Pauskar, et al. [41], introduce an averaging procedure for various families of grains as the deformation proceeds in various passes during the rolling of TMS-80R steel. Thomas, et al. [61], developed a widely used microstructure model applicable mainly for the industries based on JMAK model. This model included refinements that enables microstructure prediction for several deformation sequences as seen in forging. An applicable example would be the rolling process which is the subject of the current research. In addition, this model also expands the microstructure prediction to multiple grain families, viz, waves of recrystallized grains and the remaining ones after each deformation and subsequent holding time. This procedure is similar to the procedure mentioned in Reference [41]. The procedure developed by Thomas, et al. [61] is more comprehensive instead and has been validated for Alloy 718 which undergoes more complex microstructural processes when compared with those of the steel material considered in [41].

The formulation proposed and developed in the current work is based on the procedure developed in [61]. However, it is modified significantly to suit multi-pass rolling.

\subsubsection{Precipitates}

All these works find the average grain sizes from recrystallization alone and do not take into account the precipitation of phases such as $\gamma^{\prime}, \gamma^{\prime \prime}$ and $\delta$ that contribute essentially to the microstructural events. Gao and Wei [83] attempt to study the precipitation of $\gamma^{\prime \prime}$ while Wosik [84] and Penkalla [85] studied the precipitation of $\gamma^{\prime}$. Some researchers, as Guest et al. [57] worked with grain set models to incorporate the effects of the precipitates using Scheil's additive function. Sellars [29], discusses 
the effect of secondary phase particles on recrystallization in steel and aluminum alloys. Liu, et al. [59] observed the influence of cold rolling on the morphology and the volume fraction of $\delta$ phase of Alloy 718. However, modeling this complex precipitation requires a more detailed understanding. As noted in Section 1.3.1, the microstructure of Alloy 718 is governed by the fcc lattice structure of the $\gamma$ matrix [70] and a number of above mentioned characteristic precipitates. Above $1010{ }^{\circ} \mathrm{C}$,

only $\gamma$ phase is found in equilibrium and at temperatures below $1010{ }^{\circ} \mathrm{C}, \delta$ phase particles precipitate.

The current study does not include the influence of precipitates in evaluating the microstructure due to the complications involved in implementing the influence of precipitates into the analysis.

\subsubsection{Atomistic approaches}

Frost, et al. [81], implemented a first principles approach in predicting the grain growth using Monte-Carlo simulations. In this work, the development of crystallographic texture, the effect of dispersion of second phase particles are discussed. However, the analysis suffers from the computational intensity involved. Since it is generally difficult to relate the gross deformation behavior to atomistic and crystalline deformation mechanisms, empirical relationships have been developed for only specific ranges of temperatures, strain rates, and strains [69]. The other alternative is to employ multiscale approaches as discussed by Yu, et al. [80]. The empirical microstructural relationships are replaced by numerically efficient and accurate physicsbased models at the mesoscopic length scale. Physical laws such as the surface energy reduction law governing normal grain growth, the site-saturated nucleation law for recrystallization are employed to model the evolution of the lattice [93]. Recrystallization mapping is applied locally and then nucleus growth phase is simulated based on total energy reduction. A drawback of the multiscale approach is that the mesoscopic properties such as grain boundary energies and mobilities of various materials need to be empirically evaluated or atomistically simulated. The multiple scale approach also needs realistic initial microstructure states and orientation as well as accurate nucleation laws for recrystallization are needed. 
Due to the associated difficulties and lack of critical parameters for the material under consideration, the current work does not attempt to investigate the multiscale approach for the multi-pass rolling of Alloy 718 derivative. 


\section{CHAPTER 5: MICROSTRUCTURE ALGORITHM}

\subsection{Introduction}

In this Chapter, the formulation of the algorithm for prediction of microstructure evolution for a generic multi-stand rolling is presented. This formulation is specific to rolling processes that are modeled with flow formulations described in Chapter 2 , that is, the material is considered a viscous fluid. However, a minor modification can be applied to the developed procedure to generalize the current approach to include other formulations. In this approach, the development of microstructure variables is predicted along a streamline from the flow formulation of the rolling process during a pass. Examples of such streamlines in a pass from the FE analysis are shown in FIGURE 5.1. In addition, the microstructure is allowed to develop during interpass periods as well. However, RAWHIDE predicts only the temperature distribution during the interpass by simulating a two-dimensional transient heat-transfer analysis. Therefore, the continuation of microstructure development will be implemented along a hypothetical straight streamline whose nodes correspond to the points in time during the interpass. Typically, the points in time correspond to the locations in space since the rolled material moves during the interpass without undergoing any change in shape. Since temperature is a significant parameter that determines the microstructure and hence the properties, the accurate prediction of temperature is also critical during the pass and interpass. Hence, the need for accurate temperature history recording [75]. This has been done by modifying the existing FE code discussed in Chapter 3 to printout the calculated temperatures for use in the microstructure evolution calculations. The application of streamline outputs from commercial FE codes to calculate the evolution of material properties based on elementary rolling was implemented previously by Goerdeler, et al. [74]. Shen, [49] employs an element based approach in contrast to the proposed streamline based approach for a similar superalloy in the context of forging analysis. The following Sections describe the 


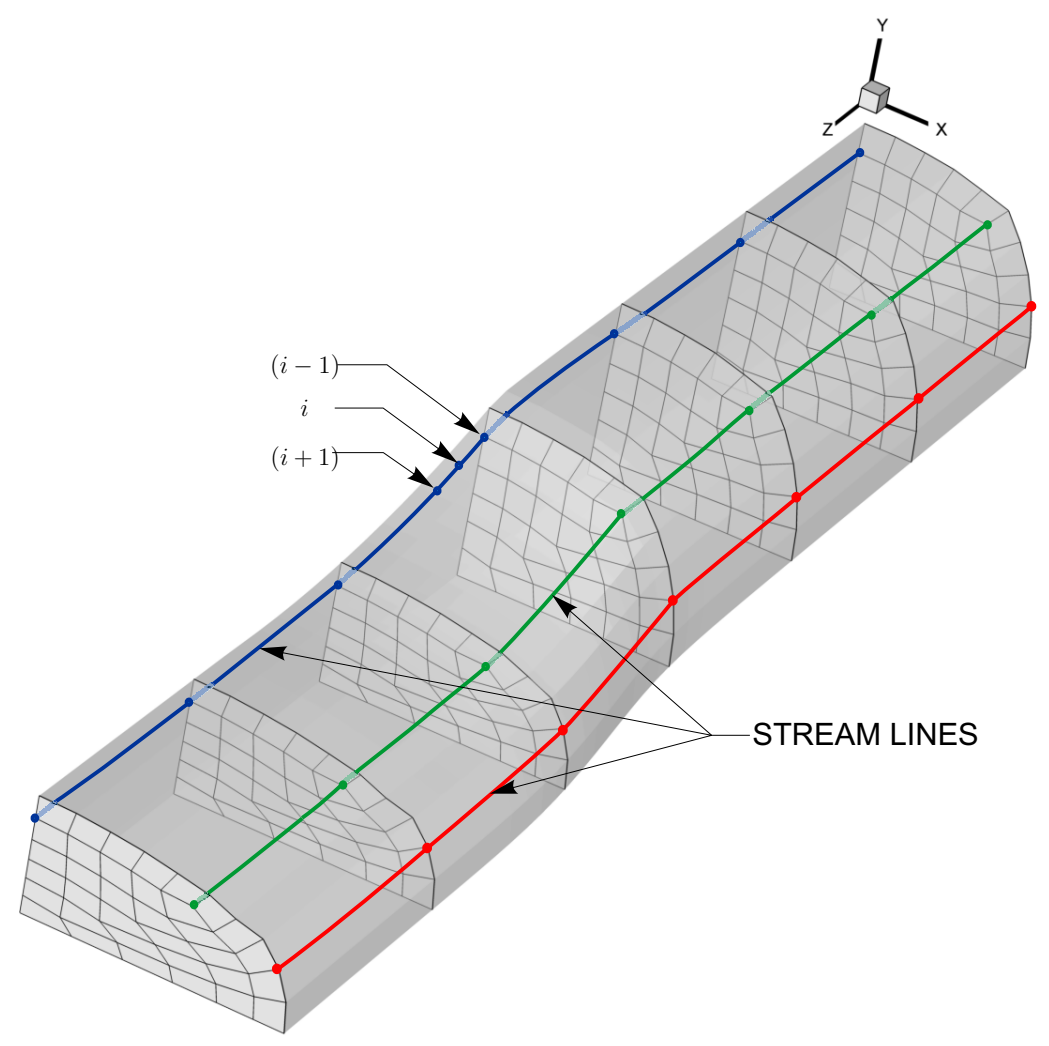

FIGURE 5.1: Typical streamlines from the 3D FE analysis (mesh from stand 3).

proposed microstructure formulation and its implementation to capture the various microstructural events described in Chapter 4. As mentioned earlier, the current approach does not include the influence of the precipitates such as $\gamma^{\prime}, \gamma^{\prime \prime}$, and $\delta$ and hence determines only the average distribution of the grain size and recrystallized fraction and hence it is applicable mostly to the supersolvus temperature regime.

\subsection{Formulation}

The proposed procedure considers two grain families; strained and recrystallized, at any given location in a streamline. This is a simplified version of the approach proposed by Thomas, et al. [61]. Both the families of grains undergo recrystallization based on a deformation criterion discussed later. The schematic shown in FIGURE 5.2 is developed based on Reference [61]. The schematic shows that an initial uniform grain size, described by $D_{s t}$, develops two grain families primarily characterized by 
the average volume recrystallized $(F)$, and average grain size $(D)$. The subscripts st and rex denote the respective strained and recrystallized family of grains. These families undergo further recrystallization based on the achievement of certain critical parameters and develop into four subgroups. The strained family develops recrystallized grains characterized by $d_{\text {strex }}, X_{s t}$ that represent the instantaneous grain size due to recrystallization laws and the recrystallization fraction respectively. The nonrecrystallized portion is the strained portion that is characterized by $d_{s t s t},\left(1-X_{s t}\right)$ which are functions of the recrystallized subgroup characteristics. Similar analogy is applicable to the recrystallized grain family subgroups and are characterized by $d_{\text {rexrex }}, X_{\text {rex }}$ for the recrystallized portion and $d_{\text {rexst }},\left(1-X_{\text {rex }}\right)$ for the strained portions. These families are expected to evolve during the deformation in a pass and during interpass. Then, an averaging algorithm is applied prior to the achievement of critical deformation parameters during the next pass. The proposed algorithm

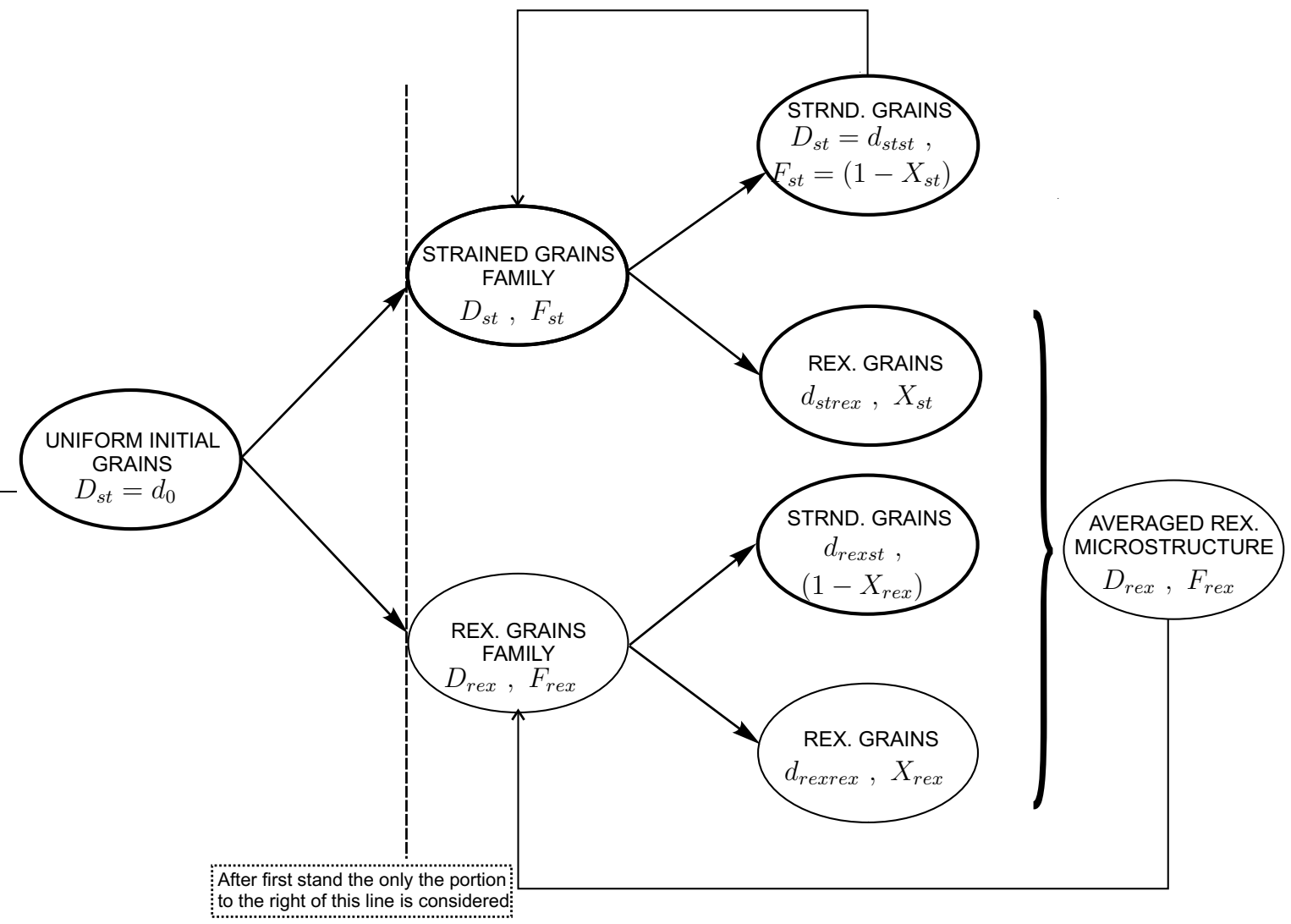

FIGURE 5.2: Microstructure families (based on Reference [61]). 
updates the following,

$$
\begin{aligned}
D_{\text {rex }} & =\frac{F_{\text {rex }} X_{\text {rex }} d_{\text {rexrex }}+F_{\text {rex }}\left(1-X_{\text {rex }}\right) d_{\text {rexst }}+F_{s t} X_{s t} d_{\text {strex }}}{F_{\text {rex }} X_{\text {rex }}+F_{\text {rex }}\left(1-X_{\text {rex }}\right)+F_{s t} X_{s t}}, \\
D_{s t} & =D_{s t}\left(1-X_{s t}\right)^{\frac{1}{n_{\alpha}}} \\
F_{\text {rex }} & =F_{\text {rex }} X_{\text {rex }}+F_{\text {rex }}\left(1-X_{\text {rex }}\right)+F_{s t} X_{s t},
\end{aligned}
$$

and

$$
F_{s t}=\left(1-X_{s t}\right) F_{s t}=1-F_{r e x}
$$

A detailed description of the microstructure evolution based on the deformation criterion can be explained with the schematic in FIGURE 5.3. The deformation during a pass is depicted with sawtooth lines at the bottom of the schematic. The figure is drawn for any arbitrary pair of stands with an interpass during continuous multi-pass rolling. The strain used for the microstructure calculation is given by equation (5.5).

$$
\bar{\varepsilon}_{x}^{i}=\bar{\varepsilon}^{i}+\nu(T) \bar{\varepsilon}_{x}^{(i-1)}
$$

where the subscript $x$ can be st or rex to represent the strained and recrystallized families, $\bar{\varepsilon}^{i}$ is the FE analysis evaluated instantaneous effective strain due to deformation, $\nu(T)$ is the temperature dependent factor used as a fraction for the previously stored strain $\bar{\varepsilon}_{x}^{(i-1)}$. The factor $\nu(T)$ varies between 0 and 1 [61]. In the present work, it is taken as a constant value of 0.5. The second term in the equation (5.5) denotes the retained strain from a previous pass. When this equivalent strain reaches a critical strain $\bar{\varepsilon}_{c}$, and if the effective strain rate $\dot{\bar{\varepsilon}}$ reaches a value greater than equal to 0.01 , DRX is initiated. Consider a single streamline shown in the FIGURE 5.4, extracted from the FIGURE 5.1. Based on the FIGURE 5.4, the strain rate is calculated as,

$$
\dot{\bar{\varepsilon}}=\frac{\delta \bar{\varepsilon}}{\delta t}
$$

that is,

$$
\dot{\bar{\varepsilon}}=\frac{\bar{\varepsilon}^{i}-\bar{\varepsilon}^{i-1}}{t^{i}-t^{i-1}} .
$$




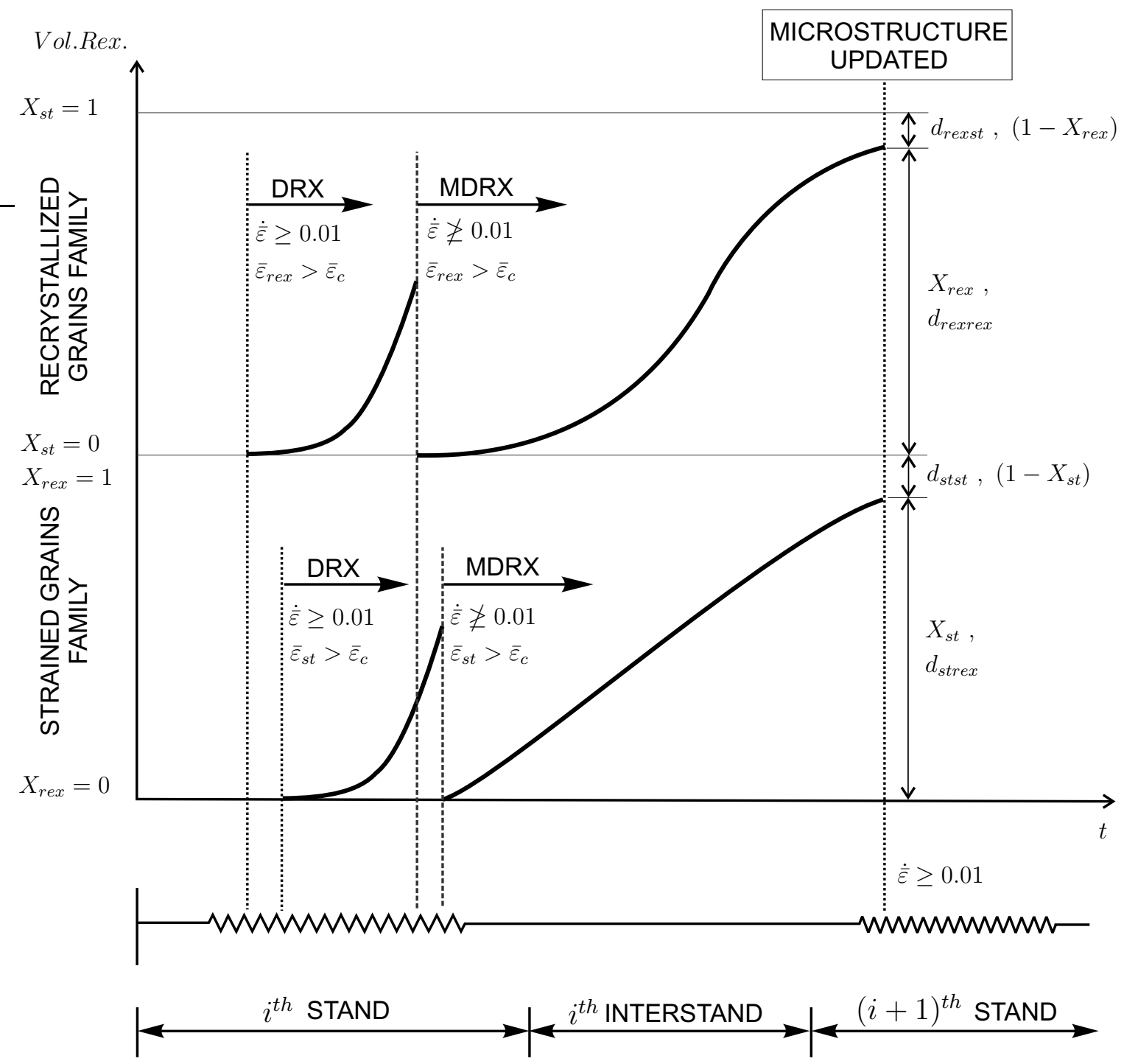

FIGURE 5.3: Microstructure evolution of different families.

MDRX follows DRX as mentioned in detail in Chapter 4 and this process does not require the the strain rate to be greater than 0.01 , however, the need for critical strain to be achieved still holds. This process assumes that the recrystallization initiates from the beginning, even though, some of the grains may be partially recrystallized due to DRX. The initial grain size used for MDRX calculations are the original, initial grain sizes used in calculating the DRX grain size. Therefore, DRX is considered only as a recrystallization initiation process and the contribution to the microstructure is primarily due to MDRX [61]. This research does not include SRX since there is no clear demarcation of MDRX and SRX in the case of Alloy 718. When the 


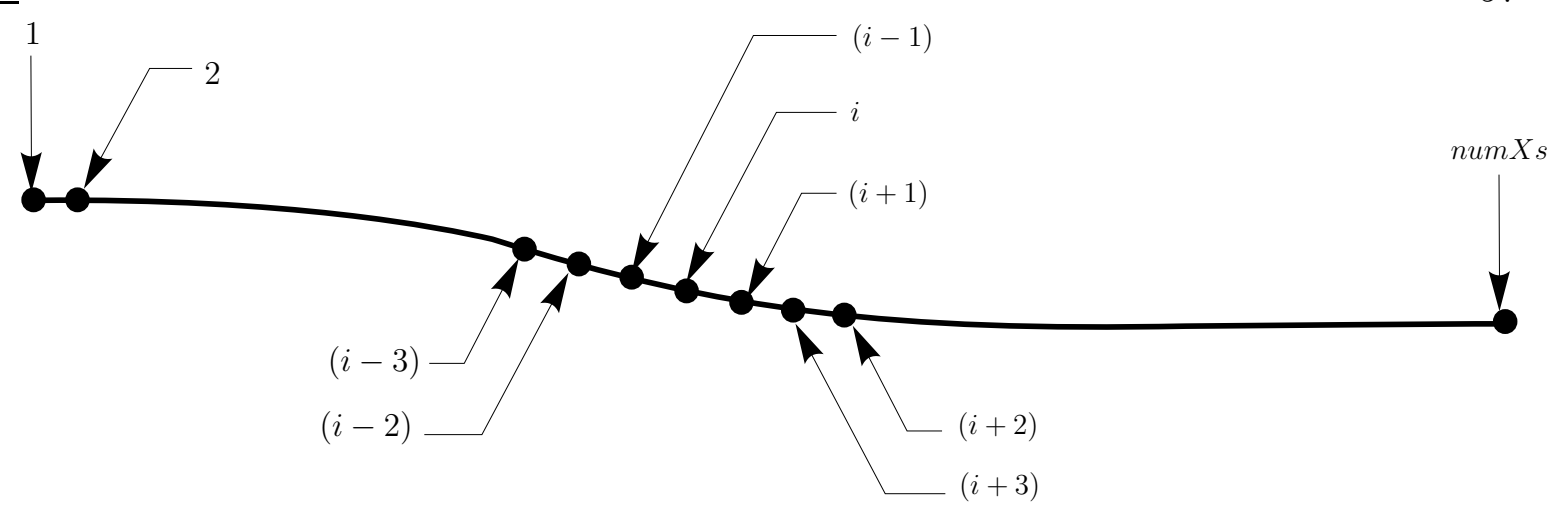

FIGURE 5.4: Nodes on a streamline.

recrystallization fraction approaches 0.98 , the applicable node is assumed to be fully recrystallized and static grain growth is initiated until further deformation changes the microstructure evolution process. In this work, the nodes in the FE mesh are considered to follow the streamline and the nodal deformation variables are used in calculating the microstructure variables.

\subsection{Implementation}

Typically, the various microstructural processes are characterized empirically based on isothermal experiments carried out at different strain rates and strains with linear regression analysis applied in developing the empirical laws [49]. Recrystallization is a continuously evolving process and an instantaneous application of the empirical laws may not capture the continuous nature of the process under consideration. In addition, the deformation is not an isothermal process. The approach described in this Chapter calculates the evolution based on time integration detailed in the following Sections. The flow chart at the end of this Chapter (FIGURE 5.7) shows the computer implementation of the detailed procedure.

\subsubsection{Dynamic recrystallization}

The rate of DRX increases with an increase in temperature and strain, and decreases with an increase in the strain rate [49;56]. A generic form of the relationship can be obtained from the literature by Shen [49], Thomas, et al. [61], and Huang, et al. [45] for superalloys. For a particular location in a streamline, this can be observed 
from the expression for fraction recrystallized expressed as,

$$
X=1-\exp \left(-\ln (2)\left[\frac{\bar{\varepsilon}}{\bar{\varepsilon}_{0.5}}\right]^{a_{d y n}}\right)
$$

where $\bar{\varepsilon}_{0.5}$ refers to the strain at which the grains are $50 \%$ recrystallized (see FIGURE 5.5). In other words, $\bar{\varepsilon}_{0.5}$ refers to the strain at which $X=0.5$ and is expressed as follows:

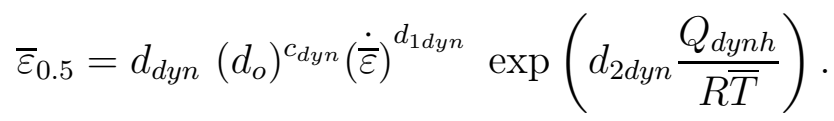

Equation (5.7) is representative of typical sigmoidal curve shown in FIGURE 5.5.

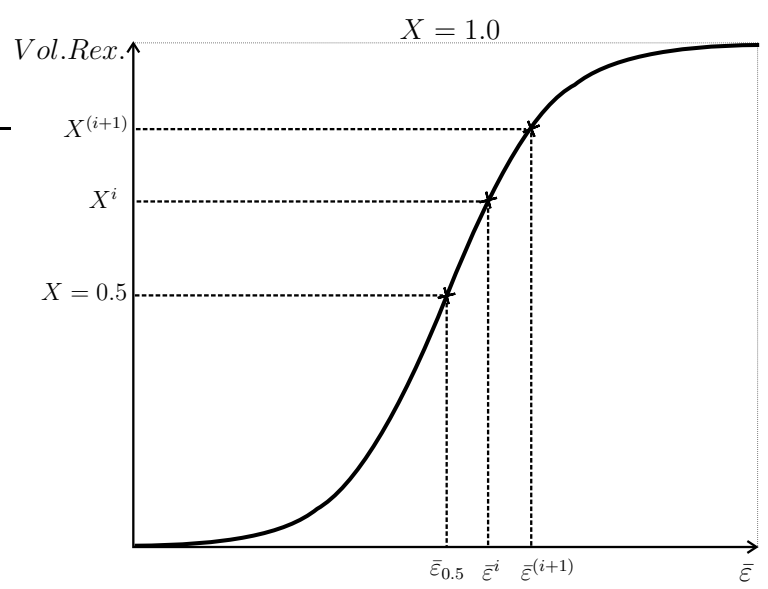

FIGURE 5.5: DRX based on strain.

In order to capture the continuous evolution of the fraction recrystallized, a time integration is necessary that incorporates the increment in strain in each increment of time. Accordingly, the recrystallization fraction for DRX is a function of the equivalent strain named as the virtual strain in this work. That is,

$$
X=f\left(\bar{\varepsilon}_{v}\right)
$$

Rewriting the equation (5.7) in line with equation (5.8),

$$
X=1-\exp \left[-\ln (2)\left(\frac{\bar{\varepsilon}_{v}}{\bar{\varepsilon}_{0.5}}\right)^{a_{d y n}}\right]
$$


or,

$$
1-X=\exp \left[-\ln (2)\left(\frac{\bar{\varepsilon}_{v}}{\bar{\varepsilon}_{0.5}}\right)^{a_{d y n}}\right] .
$$

Taking natural logarithms on both sides of equation (5.9),

$$
\ln (1-X)=-\ln (2)\left(\frac{\bar{\varepsilon}_{v}}{\bar{\varepsilon}_{0.5}}\right)^{a_{d y n}}
$$

or,

$$
\left(\frac{\bar{\varepsilon}_{v}}{\bar{\varepsilon}_{0.5}}\right)^{a_{d y n}}=\frac{\ln (1-X)}{-\ln (2)}
$$

Therefore, the virtual strain is given by,

$$
\bar{\varepsilon}_{v}=\bar{\varepsilon}_{0.5}\left(\frac{\ln (1-X)}{-\ln (2)}\right)^{\frac{1}{a_{d y n}}}
$$

During rolling, the material undergoes deformation continuously under the rolls and the strain continues to increase and so does the recrystallization fraction. In general, the recrystallization fraction for DRX during rolling is the sum of the recrystallization fraction from previous deformation and an incremental recrystallization fraction from the current deformation. This can be mathematically represented as (see FIGURE 5.4 for superscript notations),

$$
X^{i}=X^{i-1}+\delta X^{i-1}
$$

The right hand side of the above equation (5.11) can be expressed in terms of the virtual strain as,

$$
X^{i}=1-\exp \left[-\ln (2)\left(\frac{\bar{\varepsilon}_{v}^{i-1}+\delta \bar{\varepsilon}^{i-1}}{\bar{\varepsilon}_{0.5}^{i-1}}\right)^{a_{d y n}}\right]
$$

From FIGURE 5.4, initially $i=1$, therefore,

$$
\begin{aligned}
X^{1} & =X^{0}+\delta X^{0} \\
& =1-\exp \left[-\ln (2)\left(\frac{\bar{\varepsilon}_{v}^{0}+\delta \bar{\varepsilon}^{0}}{\bar{\varepsilon}_{0.5}^{0}}\right)^{a_{d y n}}\right] .
\end{aligned}
$$


Evaluating the virtual strain,

$$
\begin{aligned}
\varepsilon_{v}^{0} & =\varepsilon_{0.5}^{0}\left[\frac{\ln \left(1-X^{0}\right)}{-\ln (2)}\right]^{\frac{1}{a_{d y n}}} \\
& =0
\end{aligned}
$$

since $X^{0}$ is zero initially. Therefore, equation (5.13) simplifies to the following expression;

$$
X^{1}=1-\exp \left[-\ln (2)\left(\frac{\delta \bar{\varepsilon}^{0}}{\bar{\varepsilon}_{0.5}^{0}}\right)^{a_{d y n}}\right] .
$$

Similarly, if $i=2$, then,

$$
\begin{aligned}
X^{2} & =X^{1}+\delta X^{1} \\
& =1-\exp \left[-\ln (2)\left(\frac{\bar{\varepsilon}_{v}^{1}+\delta \bar{\varepsilon}^{1}}{\bar{\varepsilon}_{0.5}^{1}}\right)\right],
\end{aligned}
$$

where,

$$
\bar{\varepsilon}_{0.5}^{1}=f\left(\bar{T}^{i}, \dot{\bar{\varepsilon}}^{i}\right)
$$

and

$$
\bar{\varepsilon}_{v}^{1}=\bar{\varepsilon}_{0.5}^{1}\left[\frac{\ln \left(1-X^{1}\right)}{-\ln (2)}\right]^{\frac{1}{n}} .
$$

This algorithm is incorporated into GRANARY (the microstructure prediction package developed for the current work) for DRX based on the achievement of certain critical strain. The critical strain is expressed as a fraction of the peak strain. Peak strain is a material and process dependent variable. The peak strain, critical strain, and the strain corresponding to $50 \%$ recrystallization for any location are given by,

$$
\begin{aligned}
& \bar{\varepsilon}_{c}=n_{\text {dynctop }} \bar{\varepsilon}_{p}, \\
& \bar{\varepsilon}_{p}=f_{d y n} d_{o}^{g_{d y n}} \dot{\bar{\varepsilon}}^{h_{1 d y n}} \exp \left(h_{2 d y n} \frac{Q_{d y n h}}{R \bar{T}}\right),
\end{aligned}
$$

and

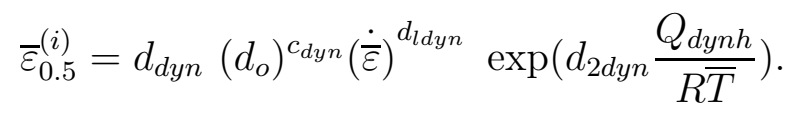


Equations (5.12), (5.14), (5.15), and (5.3.1) are evaluated by substituting the average temperature and strain rate calculated using the following expressions;

$$
\bar{T}^{i}=\frac{T^{i}+T^{i+1}}{2}
$$

and

$$
\dot{\bar{\varepsilon}}^{i}=\frac{\delta \bar{\varepsilon}^{i}}{\delta t^{i}}
$$

The grain size expression developed by empirical methods give only the grain size at steady state, that is when the recrystallization is $100 \%$ complete. However, the grain size also evolves continuously and the instantaneous grain size need to be incorporated in the recrystallization fraction [61]. Equation (5.16) is the commonly found expression for the steady state grain size due to recrystallization. Equation (5.17) is the instantaneous recrystallized grain size as a function of the fraction recrystallized. The third expression (given by equation (5.18)) denotes the grain size of the strained grains in the current family of grains.

$$
\begin{aligned}
d_{d r x}^{(s s)} & =p_{d y n}(\dot{\bar{\varepsilon}})^{q_{1 d y n}} \exp \left(q_{2 d y n} \frac{Q_{d y n d}}{R \bar{T}}\right) \\
d_{d r x}^{(i)} & =d_{d r x}^{(s s)}\left(X^{(i)}\right)^{n_{x d r x}}
\end{aligned}
$$

and

$$
d_{s t r}^{(i)}=d_{0}\left(1-X^{(i)}\right)^{n_{x d r x s t}} .
$$

In these expressions, the following parameters,

$$
\begin{aligned}
& n_{x d r x}, n_{x d r x s t}, Q_{d y n h}, Q_{d y n d}, n_{d y n c t o p}, Q_{d y n p}, a *_{d y n}, b *_{d y n}, c *_{d y n} \\
& d 1 *_{d y n}, p *_{d y n}, q 1 *_{d y n}, f *_{d y n}, g *_{d y n}, h 1 *_{d y n}, h 2 *_{d y n}, d 2 *_{d y n}, q 2 *_{d y n}
\end{aligned}
$$


are pertinent to the specific material and are described in the nomenclature. In the parameters with the symbol $*$, the

$$
\begin{aligned}
& * \text { represents } 1 \text { for sub-solvus }\left(\bar{T}<1010{ }^{\circ} \mathrm{C}\right) \\
& * \text { represents } 2 \text { for solvus }\left(\bar{T}=1010{ }^{\circ} \mathrm{C}\right) \\
& * \text { represents } 3 \text { for super-solvus }\left(\bar{T}>1010{ }^{\circ} \mathrm{C}\right)
\end{aligned}
$$

as stated in Reference [49]. As a first step to describing similar laws for Alloy 718, the empirical laws that describe the behavior of Waspaloy found in the Reference [49] were incorporated into the microstructure algorithm as one of the many models that may be utilized by the user.

\subsubsection{Metadynamic recrystallization}

When DRX is not 100\% complete, further recrystallization occur without the addition of any strain [68]. This is characterized as MDRX, as discussed in the previous Chapter. During MDRX, the recrystallization fraction is primarily a function of the time $(t)$ and a time constant $\left(t_{0.5}\right)$ at which the recrystallization is $50 \%$ complete. The general expression is similar to equation (5.7). The fraction recrystallized during MDRX and the time at which 50\% recrystallization occurs, can be expressed in general by equations (5.19) and (5.20).

$$
\begin{aligned}
X^{i} & =1-\exp \left[-\ln (2)\left(\frac{t}{t_{0.5}}\right)^{a_{m d y n}}\right] \\
t_{0.5} & =b_{m d y n} d_{o}^{c_{m d y n}} \bar{\varepsilon}^{f_{m d y n}} \dot{\bar{\varepsilon}}^{d_{1 m d y n}} \exp \left(d 2_{m d y n} \frac{Q_{m d y n h}}{R \bar{T}}\right) .
\end{aligned}
$$

The general expression for the fraction recrystallized during MDRX characterizes a sigmoidal curve shown in FIGURE 5.6 similar to FIGURE 5.5. Some literature [72] does not include time in the calculations of MDRX. This approach is questionable [56] since MDRX evolves with time without the addition of strain and strain rate does not affect the process. That is, strain rate is zero during MDRX [56]. However, the strain rate is necessary in order to calculate the variable $t_{0.5}$. In the present work, the average strain rate during deformation has been incorporated to calculate 


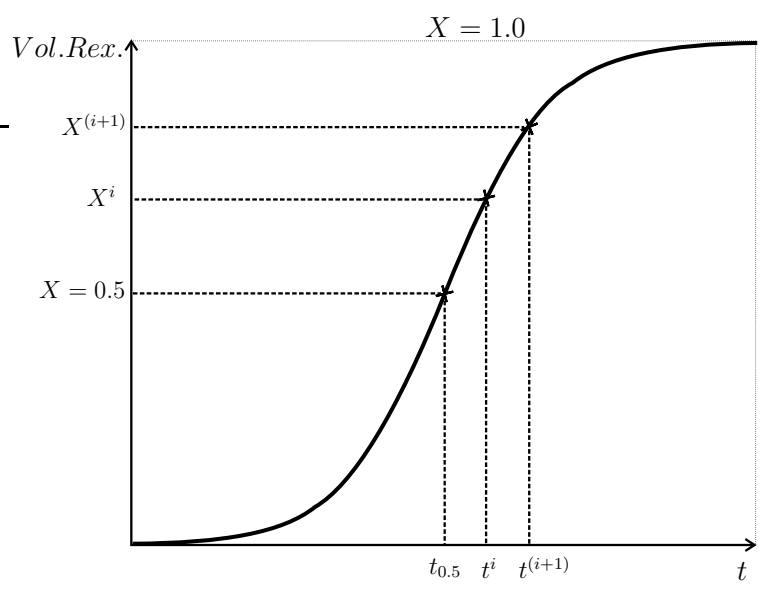

FIGURE 5.6: MDRX based on time.

this variable. To capture the continuous evolution of MDRX, a similar approach developed in the previous Section is applied in the following paragraphs. The fraction recrystallized during MDRX can be expressed in terms of a virtual time similar to the virtual strain shown in equation (5.12) as,

$$
X^{i}=1-\exp \left[-\ln (2)\left(\frac{t_{v}^{i-1}+\delta t^{i-1}}{t_{0.5}^{i-1}}\right)\right] .
$$

From FIGURE 5.6, initially $i=1$, and therefore,

$$
X^{1}=1-\exp \left[-\ln (2)\left(\frac{t_{v}^{0}+\delta t^{0}}{t_{0.5}^{0}}\right)\right] .
$$

The expression for the virtual time is similar to the expression shown in equation (5.10). That is,

$$
\begin{aligned}
t_{v}^{0} & =t_{0.5}^{0}\left[\frac{\ln \left(1-x_{o}\right)}{-\ln (2)}\right] \\
& =0
\end{aligned}
$$

Therefore, the expression for the recrystallization fraction due to MDRX simplifies to,

$$
X^{1}=1-\exp \left[-\ln (2)\left(\frac{\delta t_{0}}{t_{0.5}^{0}}\right)\right] \text {. }
$$


While predicting the microstructure evolution during MDRX, the time at which $50 \%$ recrystallization occurs and the virtual time are evaluated with the following expressions at every location in the streamline,

$$
t_{0.5}^{(i)}=b_{m d y n} d_{o}^{c_{m d y n}} \bar{\varepsilon}^{f_{m d y n}} \dot{\bar{\varepsilon}}^{d_{1 m d y n}} \exp \left(d 2_{m d y n} \frac{Q_{m d y n h}}{R \bar{T}}\right)
$$

and

$$
t_{v}^{(i)}=t_{0.5}^{(i)}\left[\frac{\ln \left(1-X^{(i-1)}\right)}{-\ln (2)}\right]^{\frac{1}{a_{m d y n}}}
$$

and substituted in the equation (5.21). The following equations for the grain sizes due to MDRX are similar to the equations (5.16), (5.17), and (5.18) for calculating the grain sizes due to DRX,

$$
\begin{aligned}
& d_{m d r x}^{s s}=p_{m d y n} d_{0}^{s m d y n} \bar{\varepsilon}^{r m d y n} \dot{\bar{\varepsilon}}^{q_{1 m d y n}} \exp \left(q_{2 m d y n} \frac{Q_{m d y n d}}{R \bar{T}}\right), \\
& d_{m d r x}^{(i)}=d_{m d r x}^{(s s)}\left(X^{(i)}\right)^{n_{x m d r x}},
\end{aligned}
$$

and

$$
d_{s t}^{(i)}=\left(1-\left(X^{(i)}\right)^{n_{x m d r x s t}}\right.
$$

and utilize the results from equation (5.21). Following are the parameters in the aforementioned expressions. These are described in the nomenclature and can be found from literature $[45 ; 49 ; 62]$ for various superalloy materials;

$$
\begin{aligned}
& a_{m d y n}, b_{m d y n}, c_{m d y n}, f_{m d y n}, d 1_{m d y n}, d 2_{m d y n}, n_{x m d r x} \\
& n_{x m d r x s t}, p_{m d y n}, s_{m d y n}, r_{m d y n}, q 1_{m d y n}, q 2_{m d y n}, Q_{m d y n h}, Q_{m d y n d}
\end{aligned}
$$

\subsubsection{Static grain growth}

When the MDRX process is $100 \%$ complete, and the material does not undergo any additional strain, annealing occurs. Any extended hold at elevated temperatures causes the grains to grow statically. There are quadratic [45] and cubic [49] laws that describe the static grain growth. A general expression that describes the static grain 
growth is expressed by equation (5.23),

$$
d_{g g r}=\left[d_{i n i}^{n_{g g r}}+t_{g g r} \delta t \exp \left(\frac{-Q_{g g r}}{R \bar{T}}\right)\right]^{\frac{1}{n_{g g r}}}
$$

where the parameters $n_{g g r}, t_{g g r}, Q_{g g r}$ are listed in the nomenclature and, as mentioned earlier, can be found from literature. The grain growth typically occurs during the long interpasses and during hold times at the end of the rolling process. 


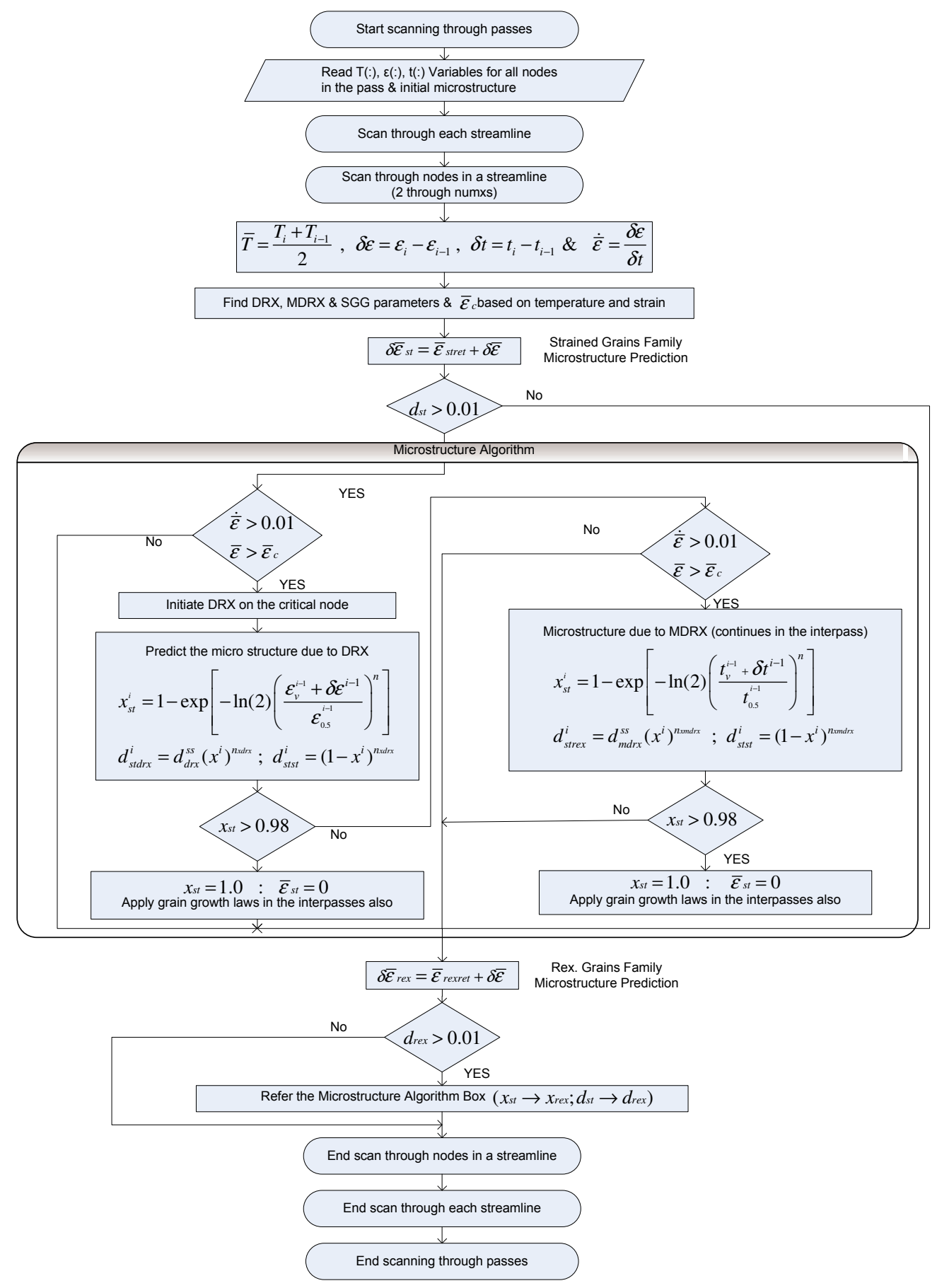

FIGURE 5.7: Microstructure algorithm. 


\section{CHAPTER 6: RESULTS AND DISCUSSIONS}

\subsection{Introduction}

The microstructure prediction algorithm was implemented in a user interactive package known as GRANARY, that can evaluate the microstructure evolution during

the multi-stand rolling of superalloys. Currently, the alloys Waspaloy [44; 49] and Alloy 718 [44; 45; 61] are included. Other superalloys may be added if the appropriate constants are known. In the present work, for the verification of the proposed algorithm, Alloy 718 was considered and a proprietary rolling pass schedule was used to carry out the FE analysis of rolling. The verification was conducted by comparing predicted microstructure to actual values for two different processes. In the first process, a 4-stand rolling followed by air cooling was considered. In the second process, a 16-stand rolling followed by air cooling and subsequent immersion quenching in water was considered.

The mesh considered for the rolling and microstructure analysis was developed with 5 core divisions and 1 outer division (see FIGURE 6.1(a)). The details of the core and outer divisions are given in Section 2.5 in line with FIGURE2.4(a). FIGURE 6.1(a) also shows some locations with symbols $\boldsymbol{\bullet}, \boldsymbol{\nabla}, \mathbf{\square}$, and $\boldsymbol{\Delta}$ chosen to study the history of microstructure evolution over many stands. These locations characterize center, mid radius, sub surface and surface locations, chosen to permit a comparison of predicted microstructure results with the experimental observations.

Even though the FE algorithm can accommodate a finer mesh, it was observed that the chosen mesh, that is, 5 core and 1 outer division, gives accurate enough results when compared with the measured temperature and shape at intermediate and final stands. It should also be noted, that the calculations are carried out at each node in a cross section and each node constitutes a point on a streamline. This approach is different from the approach found in the Reference [49], where microstructure was evaluated by using a nodal variables averaging in order to predict the average grain 
size of a particular FE mesh element. Since the deformation variables are extracted

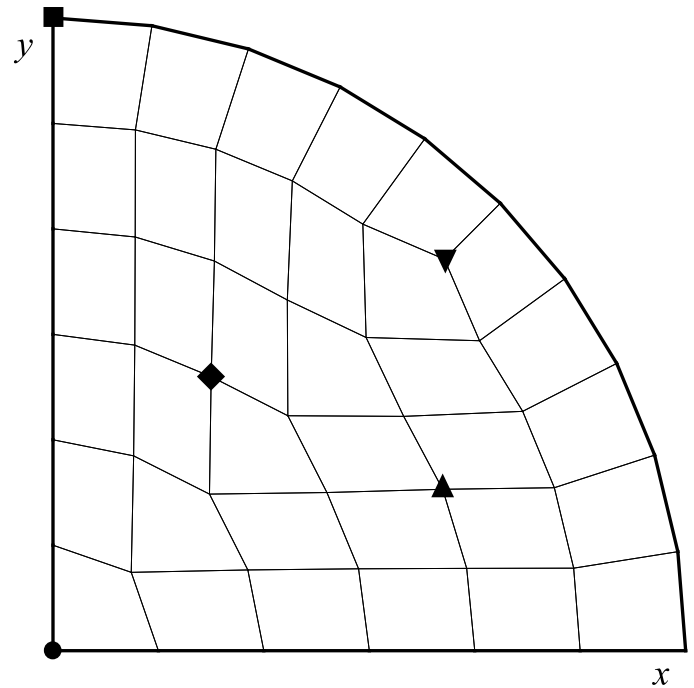

(a) Mesh and benchmarking locations.

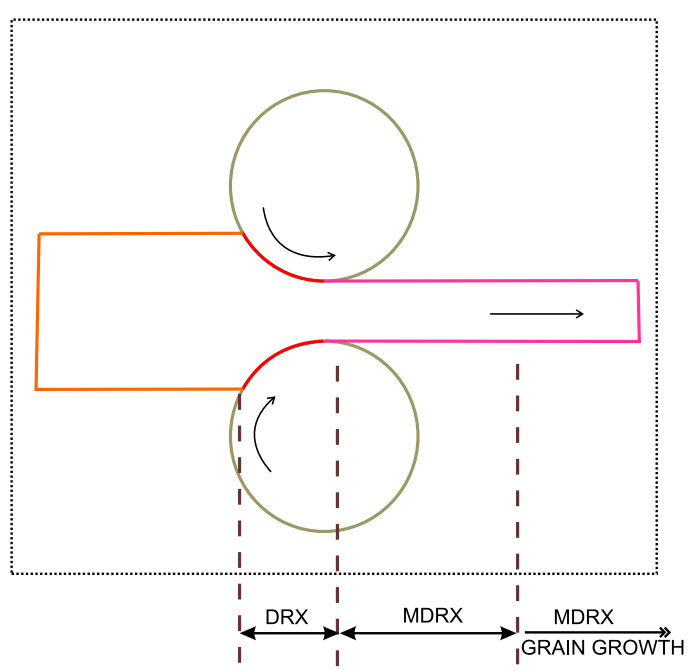

(b) Microstructure control volume.

FIGURE 6.1: Mesh considered and the schematic control volume in a pass.

to the nodes from the integration points, it was chosen to use the nodal variables to predict the microstructure and since the accuracy is not significantly affected by choosing the mesh considered, the selection of the mesh is justified. An initial uniform grain size of $90 \mu \mathrm{m}$ (ASTM rating 4, [92]) is used as input to the analysis.

\subsection{Cooling}

At the end of rolling, the bars may be either air cooled or water quenched. Water quenching can be with water sprays or by immersion. The current algorithm incorporates a cooling analysis at the end of the desired stand that is either air cooling, water quenching or a combination. At high strain rates, quench time becomes a critical parameter due to MDRX [61]. In addition, the micrographs to compare the predicted results were captured at the end of $4^{\text {th }}$ stand with an assumed 5 Seconds air cooling and at the end of $16^{\text {th }}$ stand with 2 Seconds air cooling and 2 Seconds immersion water quenching. Therefore, the results comparison incorporates both the analyses and involves a detailed discussion of the evolution. As a brief review of the discussion made in the previous Chapter, FIGURE 6.1 gives a larger picture of the 
microstructure evolution process. That is, DRX process under the rolls as deformation continues and MDRX in the region close to the rolls and a continuation of the MDRX and a static grain growth during the interpass or cooling depending on the achievement of $100 \%$ recrystallization.

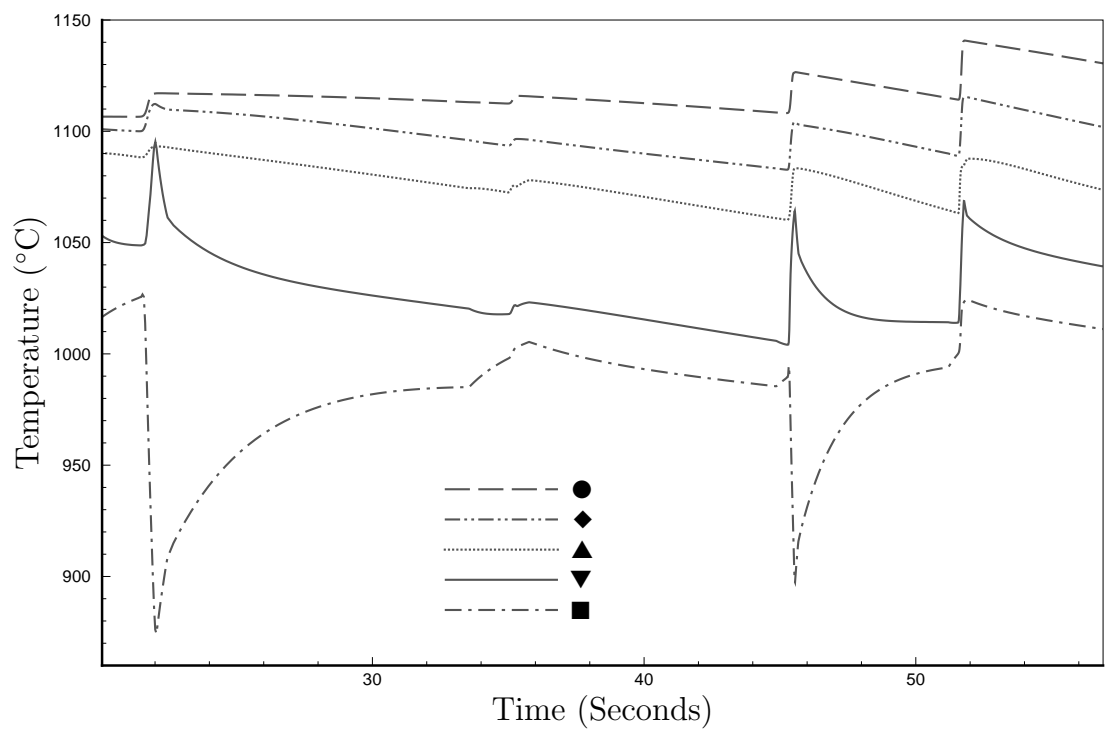

FIGURE 6.2: Temperature history on various streamlines (4-stand analysis).

\subsection{4 -Stand analysis with air cooling at the end of $4^{\text {th }}$ stand}

The temperature and equivalent strain histories at the chosen locations are shown in FIGURES 6.2 and 6.3 respectively. From these figures, it can be observed that the strains are low in the initial two stands, specifically very low during the $2^{\text {nd }}$ pass with a maximum value of 0.25 . It should be noted that, in FIGURE 6.3, the independent axis is not to scale. That is, the interpass times are much bigger compared to the pass times and hence the figure was created with actual strains and not to scale times. The strains reach a maximum of 0.9 during the $4^{\text {th }}$ pass at the subsurface location represented by the symbol $\boldsymbol{\nabla}$. The temperatures (FIGURE 6.2) are always maximum at the center location (represented by the symbol 0 ) and always minimum at the outer surface (represented by the symbol $\boldsymbol{\Delta}$ ). This is the expected pattern of deformation heating, that is, the core heats up more than the surface since there are radiative 


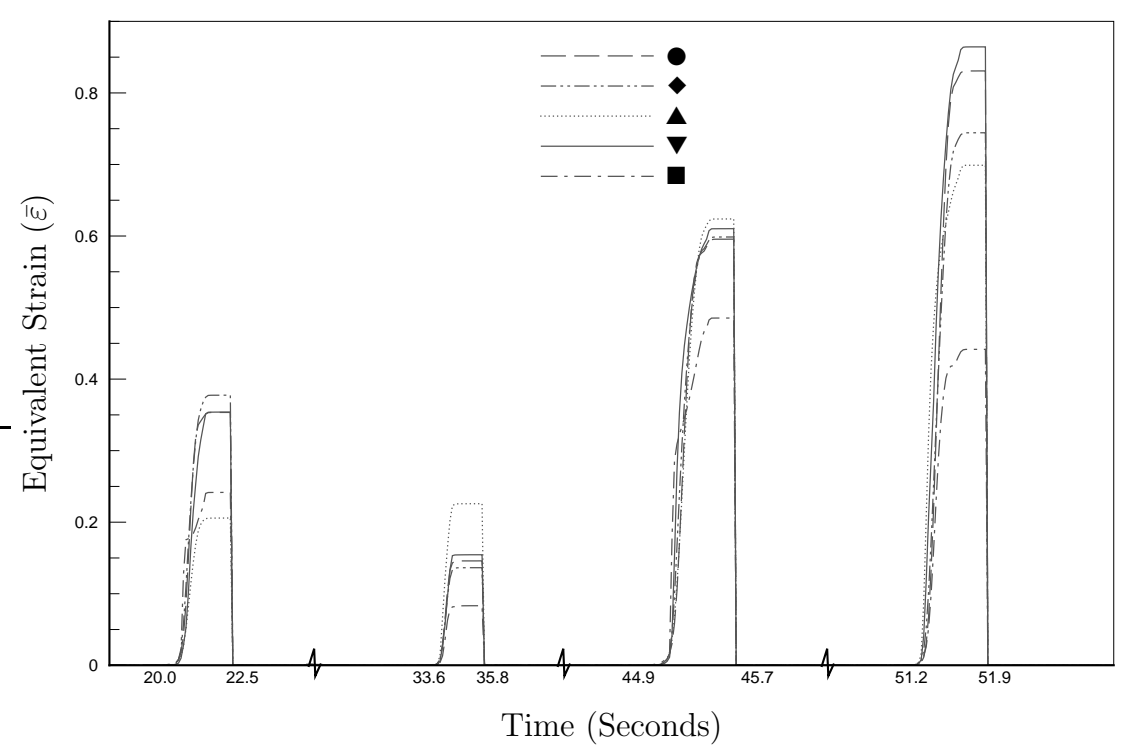

FIGURE 6.3: Strain history on various streamlines (4-stand analysis).

and convective heat losses at the surface. From FIGURE 6.2, the interpass regions in general reduce the temperature in the internal locations whereas the surface location represented by the symbol $\boldsymbol{\square}$ heats up.

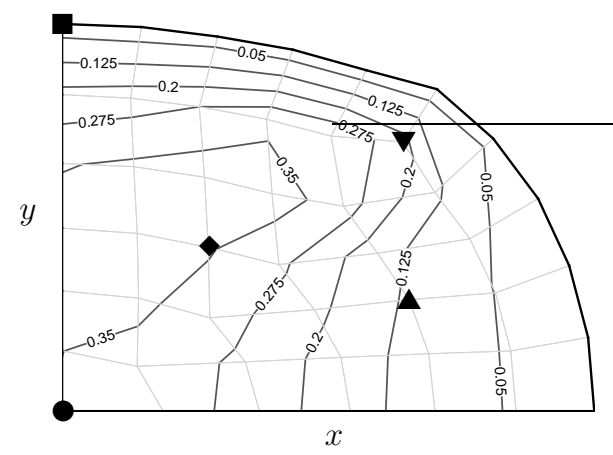

(a) $X_{s t}$ at the end of Stand-1.

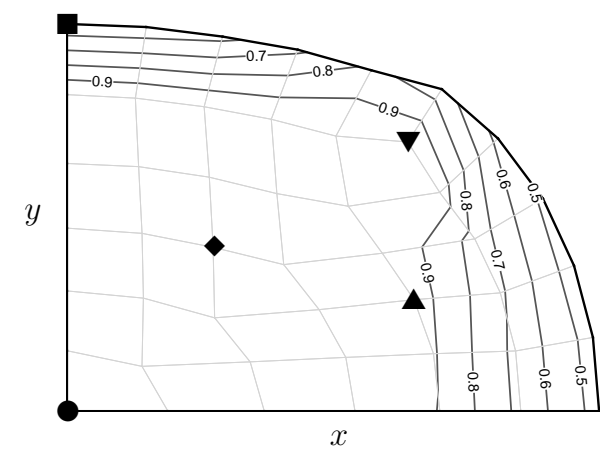

(b) $X_{s t}$ at the end of Interstand-1.

FIGURE 6.4: Recrystallization fractions (x100\%) at the end of $1^{\text {st }}$ stand and insterstands. 


\subsubsection{Initial observations}

The initial grain size denoted as $d_{0}$ is the initial strained grain family's grain size $\left(D_{s t}\right)$ and obviously, all the grains are initially strained grains only. That is, $F_{r e x}$ is 0 , and hence $F_{s t}$ is equal to 1.0. FIGURES 6.4(a) and 6.4(b) indicate the recrystallized fraction of the strained families. The grains close to the center are more recrystallized at the end of Stand-1 and at the end of interstand-1. This is due to the fact, that the strains (FIGURE6.3) and temperatures (FIGURE6.2) are higher near the core than at the other locations in the cross section. The recrystallization is primarily due to MDRX immediately after the deformation and during interpass. At the end of first stand, in the central region, initial grains are almost fully recrystallized while the surface experienced partial recrystallization. At the end of $2^{\text {nd }}$ stand, there are two families of grains, the strained and the recrystallized families. Therefore, the recrystallization fraction was evaluated for both the families. FIGURES 6.5(a)

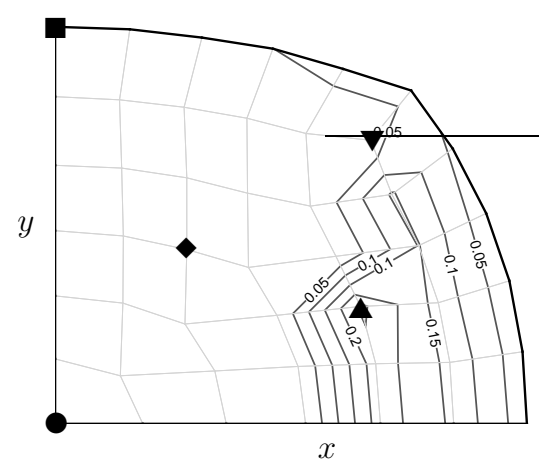

(a) $X_{s t}$ at the end of Stand-2.

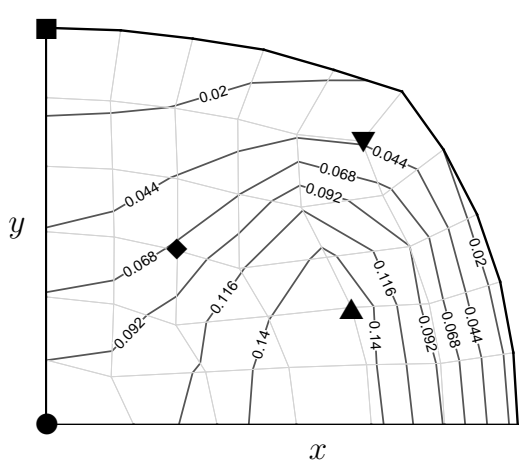

(b) $X_{\text {rex }}$ at the end of Stand-2.

FIGURE 6.5: Recrystallization fractions (x100\%) at the end of $2^{\text {nd }}$ stand.

and $6.5(\mathrm{~b})$ indicate the fraction recrystallized for strained and recrystallized families respectively. Since the strains attained during the $2^{\text {nd }}$ pass are very low as observed in FIGURE 6.3 for specific locations and in FIGURE 6.6(a) for the entire cross section, the recrystallization is very low for both families. However, high temperature (see 
FIGURE 6.6(b)) at the end of the pass and the interpass drives the MDRX for the recrystallized family and as high as $80 \%$ recrystallization is achieved in that family of grains as observed in FIGURE 6.7(b). A portion of the strained family, specifically represented by the location $\boldsymbol{\Delta}$ is also recrystallized as high as $98 \%$ (see FIGURES $6.7(\mathrm{a})$ and 6.10).

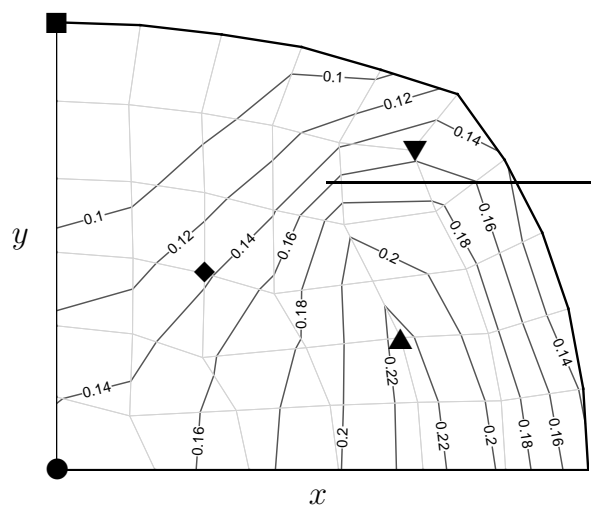

(a) Effective strain $(\bar{\varepsilon})$.

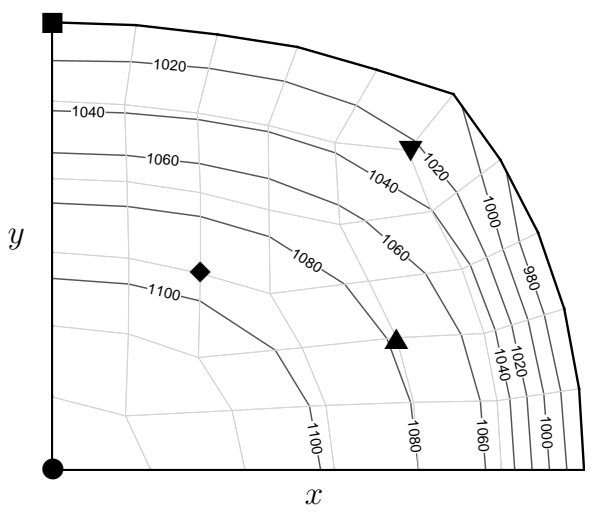

(b) Temperature ${ }^{\circ} \mathrm{C}$.

FIGURE 6.6: Deformation variables from the FE analysis at the end of $2^{\text {nd }}$ stand.

\subsubsection{Streamline results}

Some of the initial observations indicate that the microstructure algorithm captures the real behavior of the material under consideration fairly accurately. However, the verification of the algorithm can be accomplished effectively by observing the microstructure history along the streamlines indicated by the locations shown in FIGURE 6.1(a).

FIGURES 6.8 through 6.12 give a clear picture on the effectiveness of the proposed and implemented microstructure algorithm. These figures have three subfigures; (a) showing the variation of a normalized temperature and normalized strain over the time, (b) showing the recrystallization fractions and normalized microstructure variables for the recrystallized family of grains over time, and (c) showing the recrystallization fractions and normalized microstructure variables for the strained family of 
grains over time. Normalization of some of the variables enable the visualization of many variables in the same figure. The subfigures indicated by (a) is a repetition of FIGURES 6.2 and 6.3, however, normalized. The strains in subfigures represented by (b) and (c) indicate the assumed strain till the recrystallization continue. Therefore, in many of the cases, the strains represented for two families of grains last a little longer than the applied instantaneous strain. When the recrystallization is complete, that is $98 \%$ of the grains in any family, the strains corresponding to that family is set to zero until further deformation increases the strain.

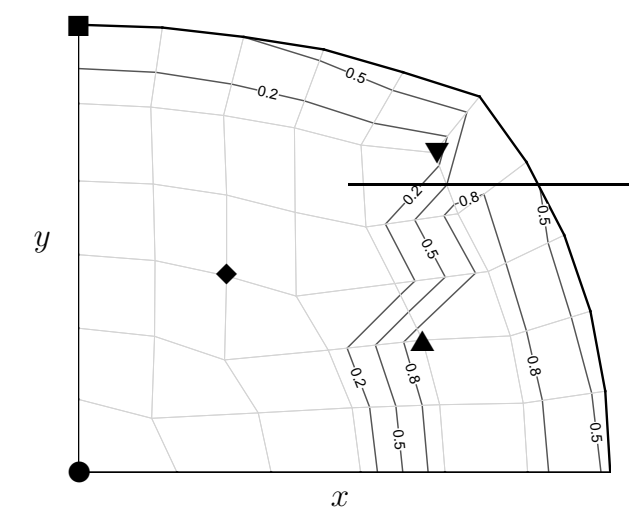

(a) $X_{s t}$ at the end of Interstand-2.

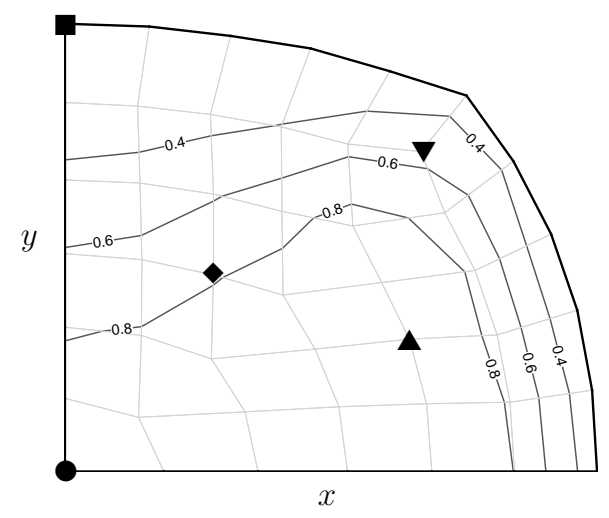

(b) $X_{\text {rex }}$ at the end of Interstand-2.

FIGURE 6.7: Recrystallization fractions (x100\%) at the end of $2^{\text {nd }}$ interstand.

At the central location (see FIGURE 6.8), recrystallization initiates with DRX and continues with MDRX as soon as the addition of the strain stops, and progresses till the recrystallization is complete during the interpass. Strains are reset to zero for the strained family and the grains start to grow during the interpass. Prior to the achievement of the strain rate and critical strain condition in the second pass, the microstructure averaging algorithm updates the microstructure and as seen in the figure, $F_{s t}$ is set to zero since all the grains at this location are fully recrystallized.

During the deformation in the $2^{\text {nd }}$ stand, the recrystallized family undergoes recrystallization and the MDRX does not recrystallize the REX family of grains fully. 
However, due to the high strains and temperatures in Stand-3, the MDRX completes and a similar phenomenon is observed during Stand- 4 also. Therefore, at this location, at the end of $4^{\text {th }}$ stand, only recrystallized family exists and the grain size is approximately $70 \mu m$.

Similar characteristics are observed for the locations represented by $\bullet$ and $\boldsymbol{\nabla}$, however, a rapid grain growth is observed at the location represented by $\boldsymbol{\nabla}$. The grain sizes are approximately $55 \mu \mathrm{m}$ and $20 \mu \mathrm{m}$ at the respective locations. FIGURES 6.9 and 6.11 indicate the observations clearly. From FIGURE 6.10, for the location indicated by the symbol $\boldsymbol{\Lambda}$, the strained family of grains vanish only at the end of $2^{\text {nd }}$ stand. On the other hand, from FIRURE 6.12, it takes four stands for the surface location indicated by the symbol $\mathbf{a}$ to recrystallize completely.

\subsubsection{Final observations}

The microstructure evolution for specific streamlines clearly depict the effective implementation of the proposed and developed algorithm shown schematically by FIGURE 5.3. A contour plot of the recrystallized fraction $\left(F_{r e x}\right)$ at the beginning of the deformation during the $4^{\text {th }}$ stand and the recrystallized fraction $\left(X_{\text {rex }}\right)$ at the end of the air cooling analysis after the $4^{\text {th }}$ stand are depicted in FIGURES 6.13(a) and 6.13(b) respectively. $F_{\text {rex }}$ is calculated based on the averaging algorithm proposed in Section 5.2 and given by equation 5.3. It can be observed that there are very few portions in the cross section near the surface that are not fully recrystallized.

An observation on the grain sizes due to FIGURES 6.14(a) and 6.14(b) indicate that the region near the core experienced a significant grain growth due to the high temperatures while the surface regions show smaller grains. FIGURE 6.15] indicates the observed microstructure at various locations and the grain sizes at those locations. It is clear that the grains show signs of complete recrystallization at all locations and groups of recrystallized families as seen in FIGURE 6.15(c). Also, at the center (FIGURE 6.15(a)) the grains are larger and smaller at the midradius location (FIGURE 6.15(b)) while they are smaller at the surface (FIGURE 6.15(c)). 


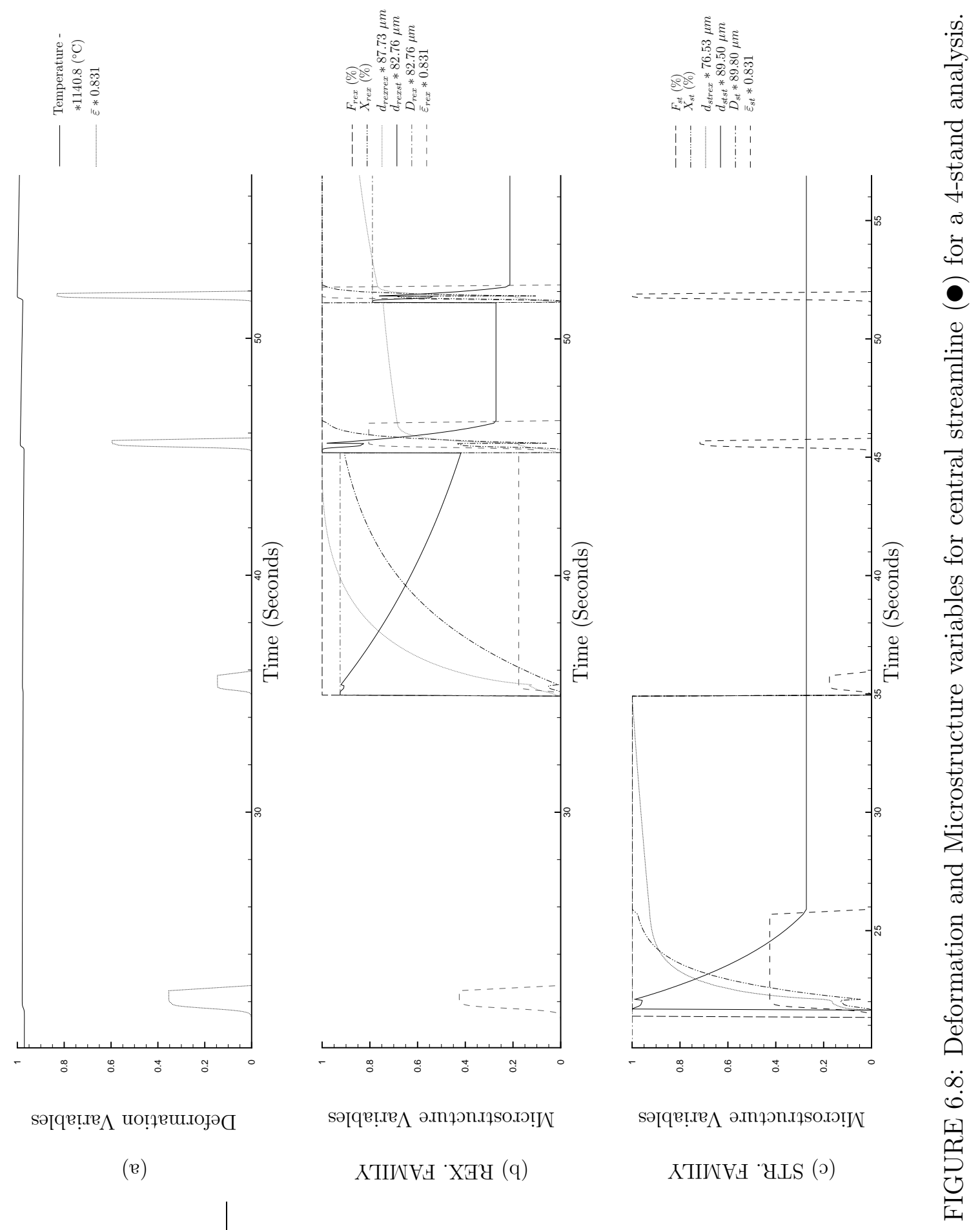




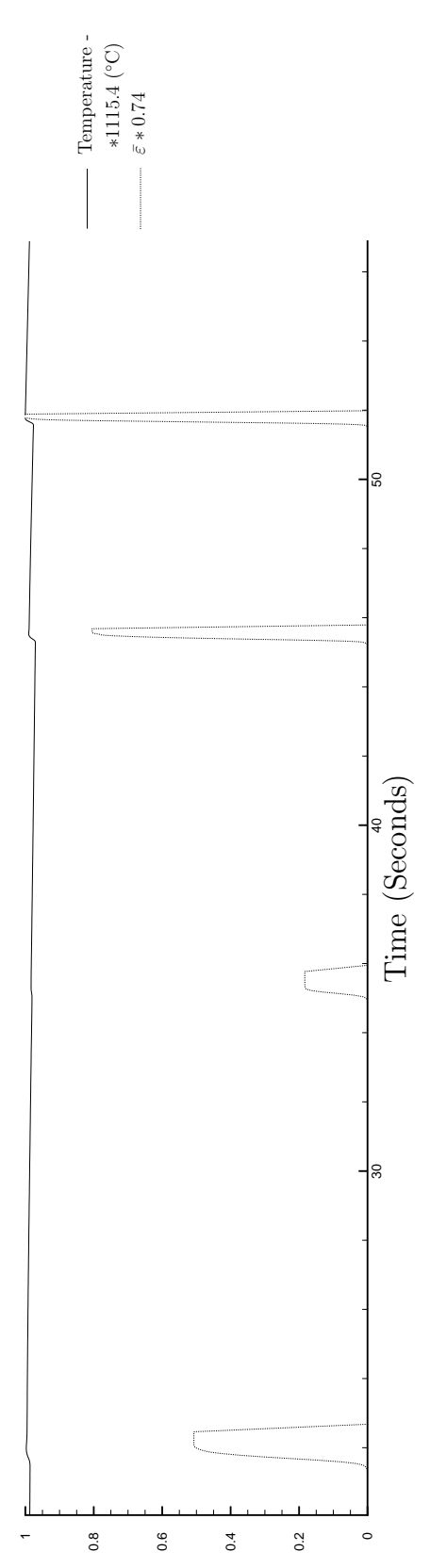

sәгqетฺе $\Lambda$ иотұешлојә

(e)

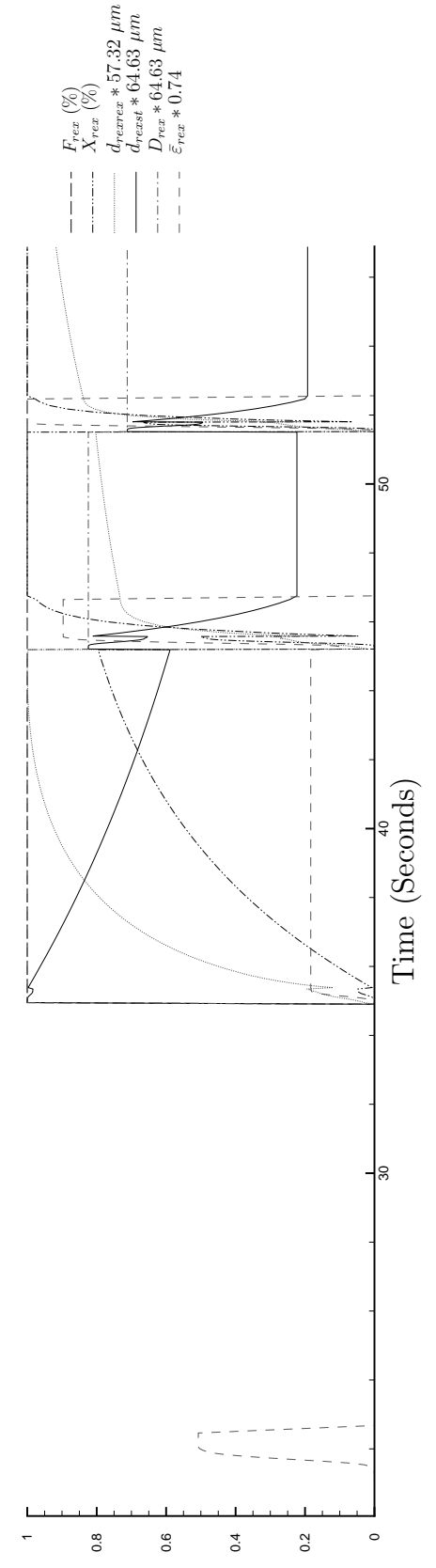

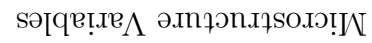

ХTINVН ХӒЧ (q)

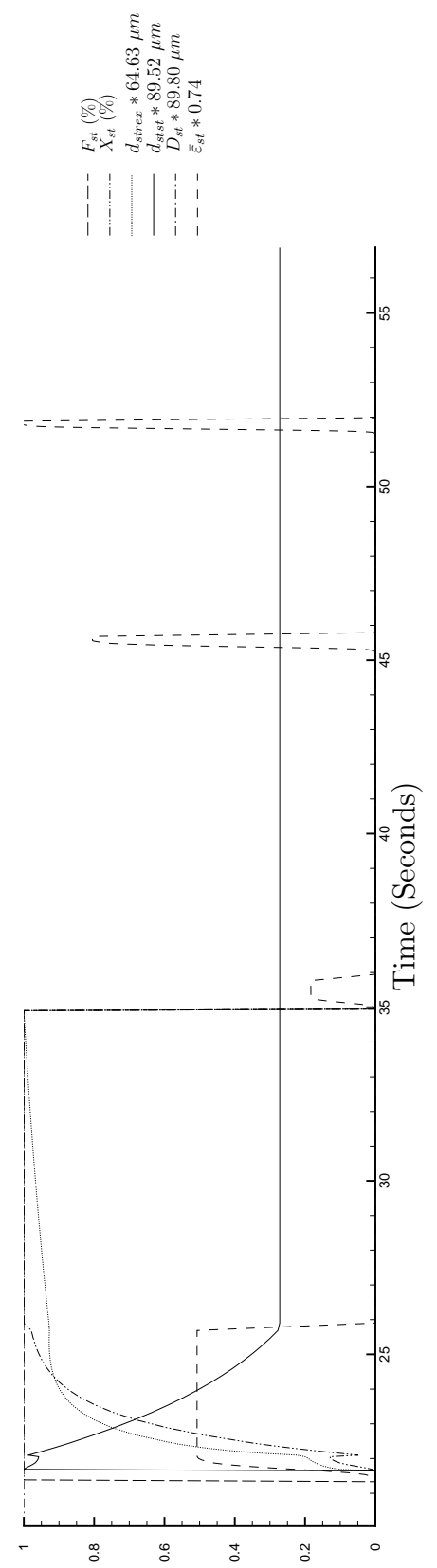

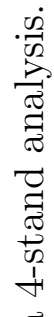

¿ิ

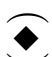

.

to

घ

.

$\frac{\mathscr{d}}{\frac{0}{\sigma}}$

$>$

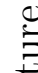

0
0
0
0
0
0
0
0
0
0
0
0
0
0
0
0
0
0
0
0
0

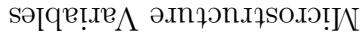

XTINVH ' $\mathrm{GLS}$ (ว) 


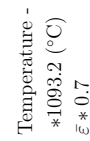

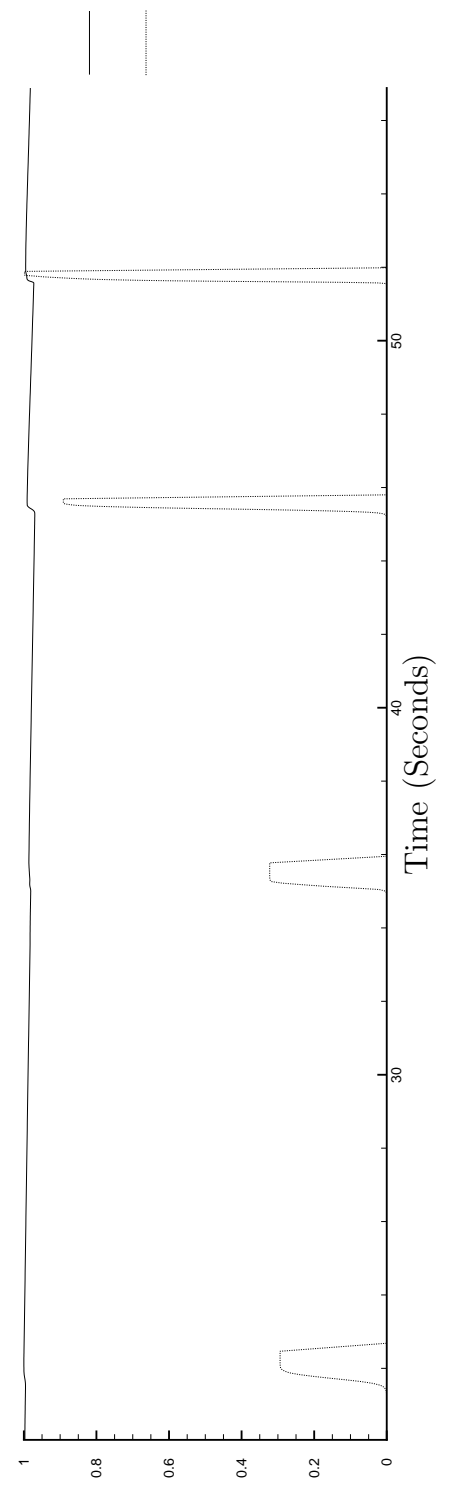

sәгqетฺе $\Lambda$ иотұешлојә

(२)
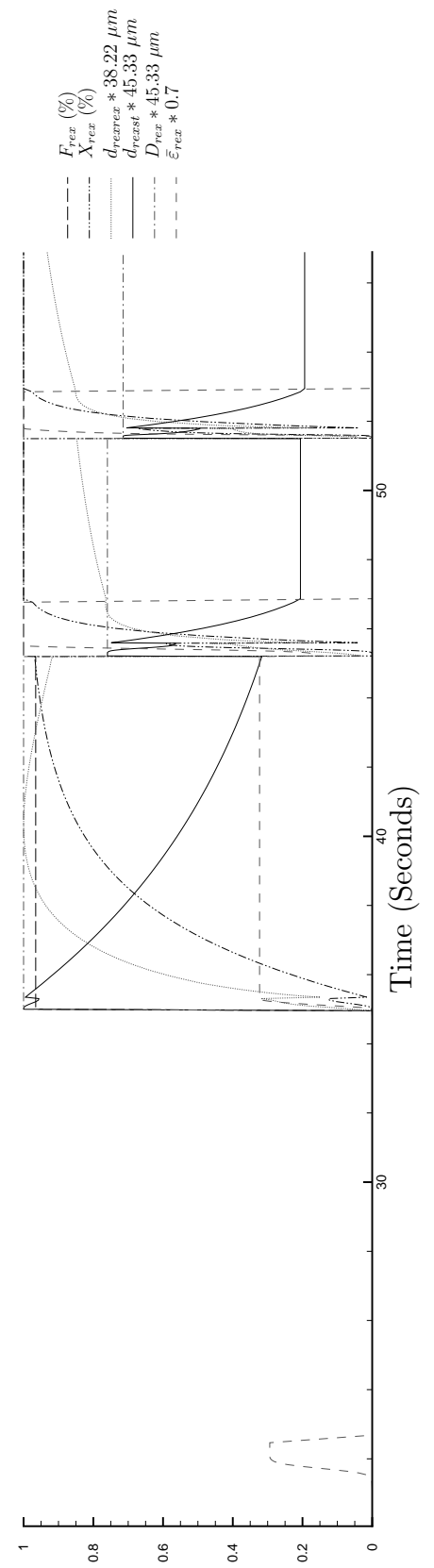

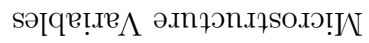

ХTINVН ХӒЧ (q)

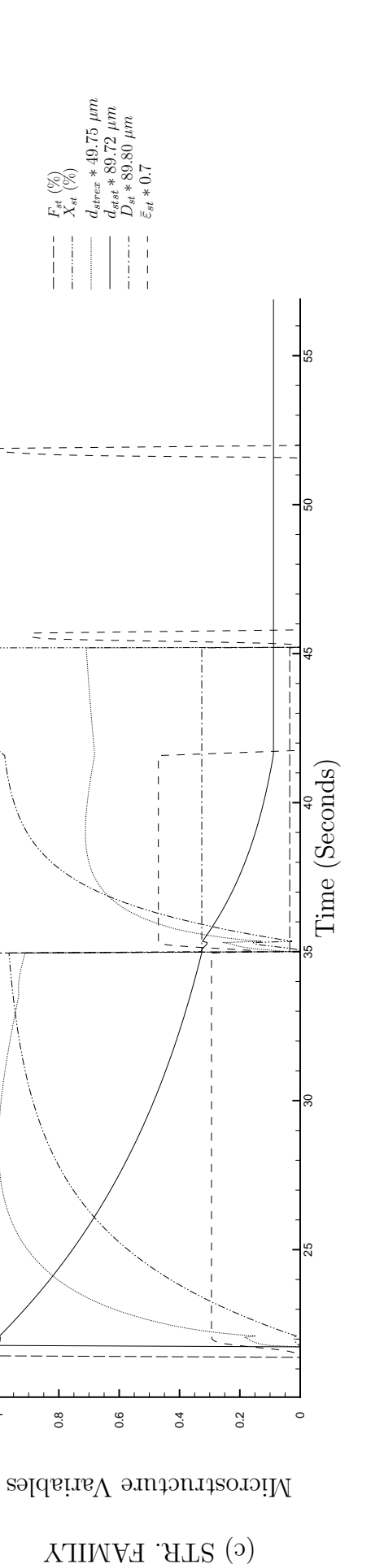

$\cdot \frac{\dot{2}}{\pi}$

㻤

륨

$\frac{1}{4}$

$\approx$

อ 


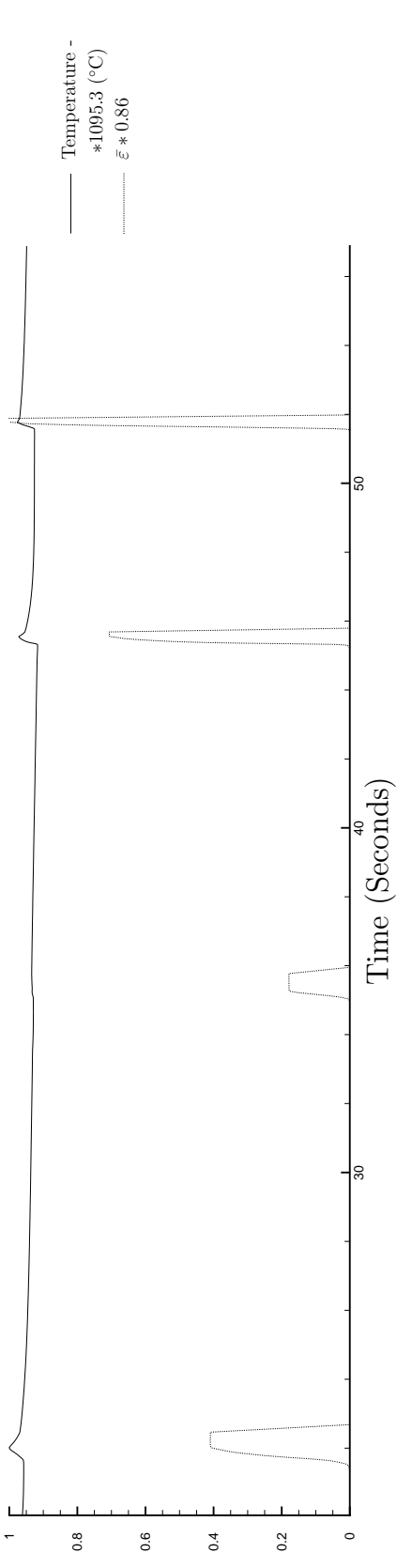

sәгqетฺе $\Lambda$ иотұешлојә

(e)

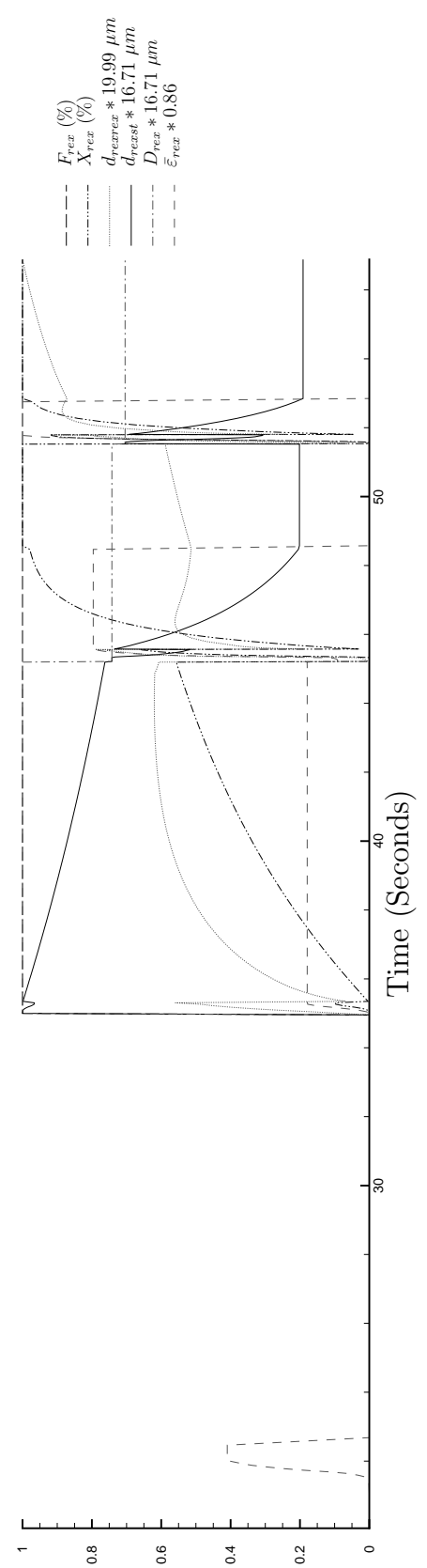

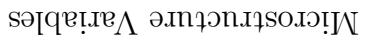

ХTIN⿴H ХӒЧ (q)

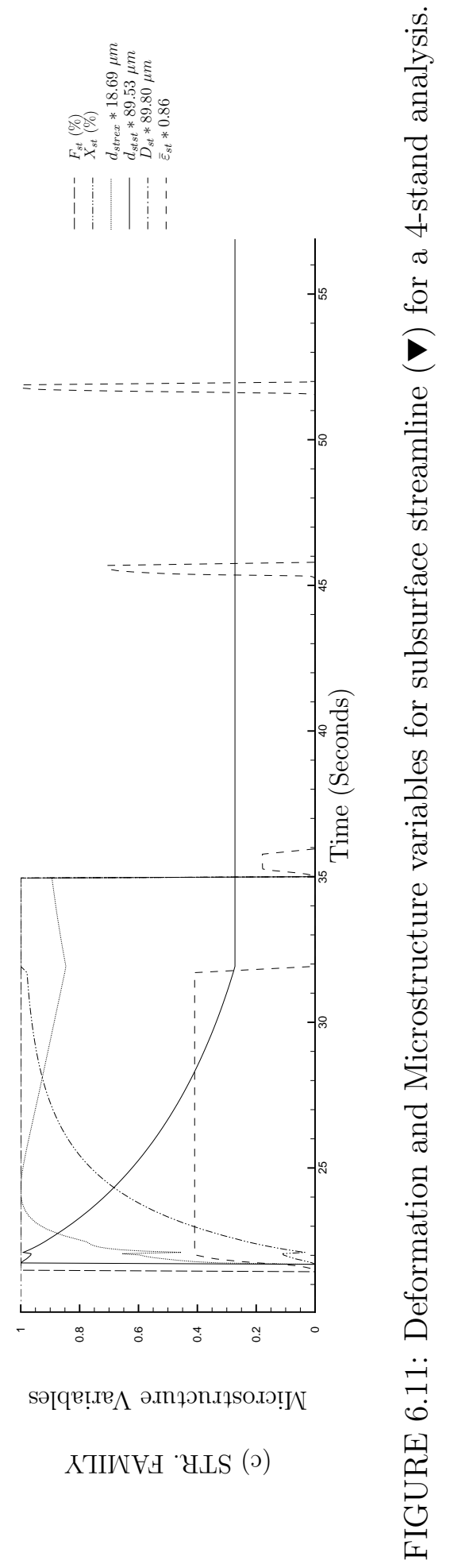




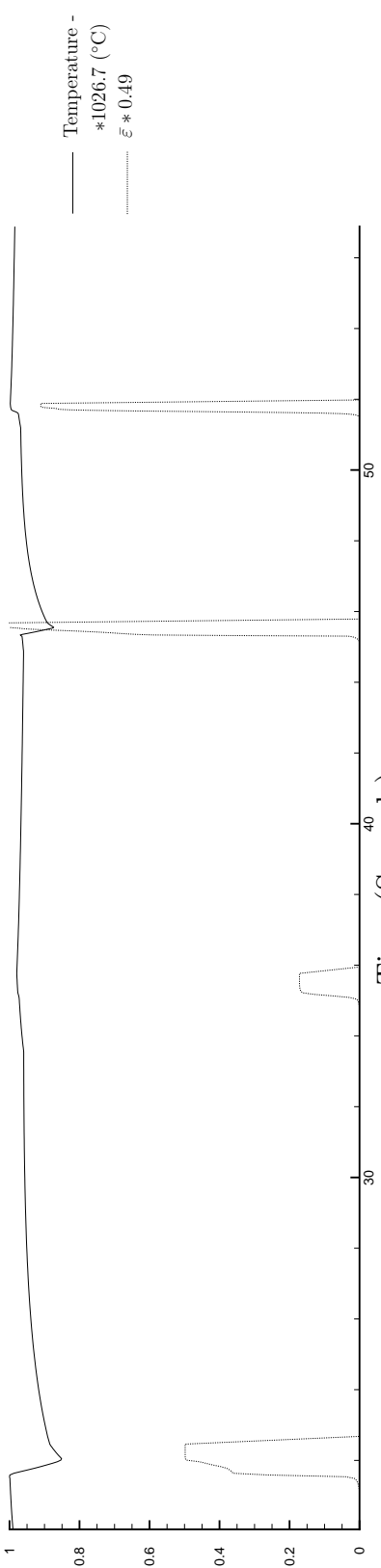

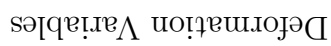

(e)

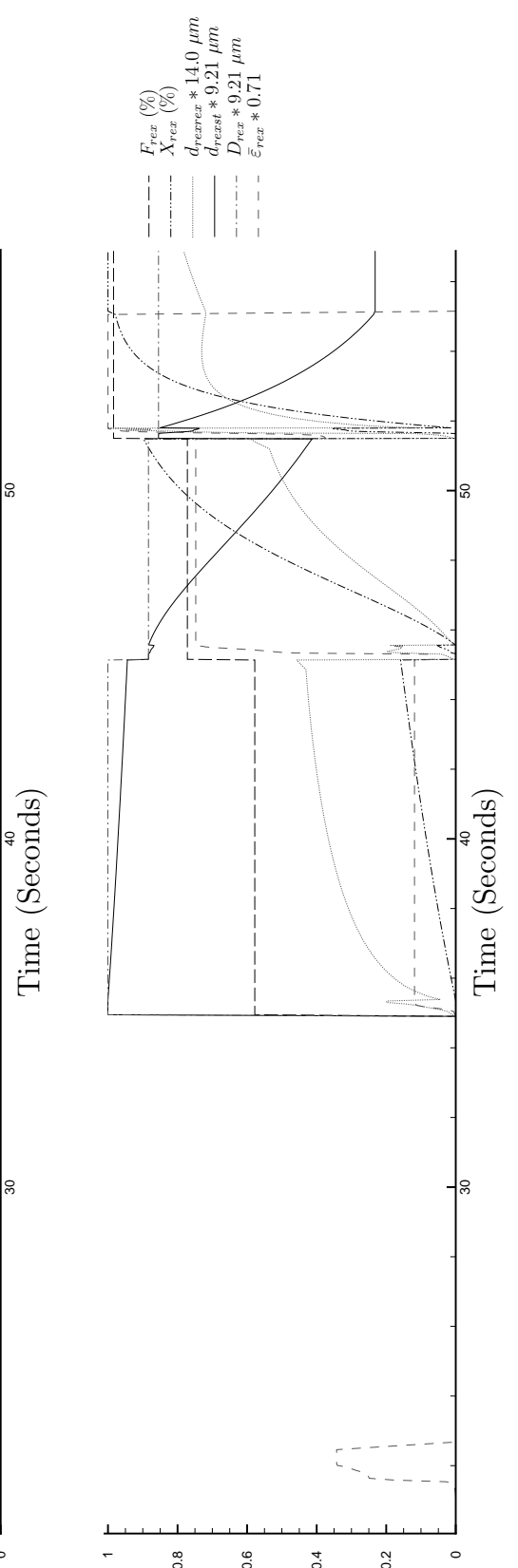

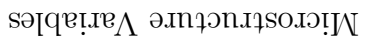

ХTINVH ХЭЧЧ (q)

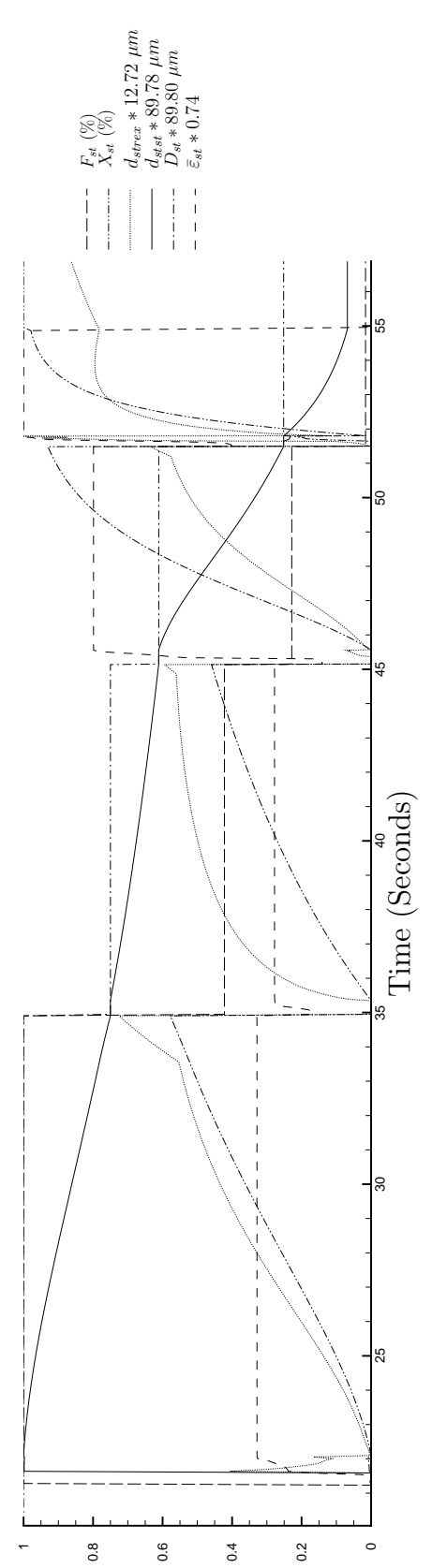

完

0
$\frac{\pi}{\pi}$
$\frac{\pi}{0}$
$\frac{1}{4}$
+

ఠ

ఠั

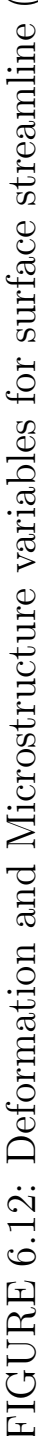




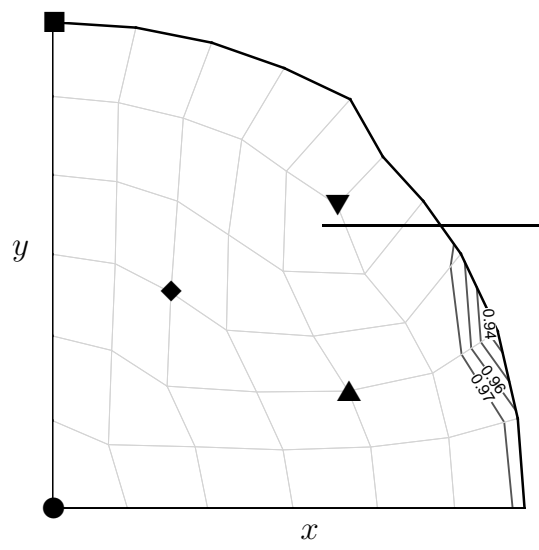

(a) $F_{r e x}$ at the beginning of Stand- 4 .

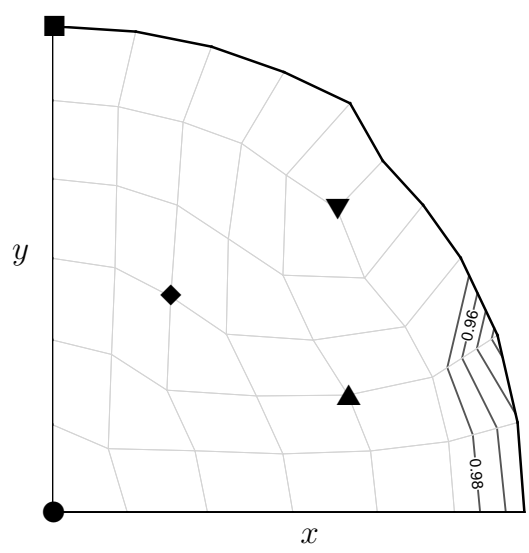

(b) Fraction Rec. $X_{\text {rex }}$ at the end of cooling.

FIGURE 6.13: Recrystallization Fractions (x100\%) during the $4^{\text {th }}$ stand and after air cooling after $4^{\text {th }}$ stand.

6.4 16-Stand analysis with air cool and water quenching after $16^{\text {th }}$ stand The microstructure evolution algorithm is extended for evaluating the microstructure at the end of a 16-stand rolling analysis. Since the mesh distortion issues are previously addressed by a mesh-rezoning procedure developed in Chapter 3, the microstructure evolution can be analysed for as many stands as the real industrial mill can have. Since there is a deformation variables interpolation involved during meshrezoning procedure, the same approach was incorporated in the microstructure evolution algorithm also.

GRANARY encompasses the interpolation algorithm in addition to the microstructure evolution algorithm, and cooling analysis algorithm. In the case of the 16-stand analysis, the cooling at the end of the $16^{\text {th }}$ stand is carried out in two steps. Firstly, an air cooling for 2 Seconds that captures the time for transferring the billet, and an additional 2 Seconds that captures the evolution during immersion water quenching. It is clear from the observations based on FIGURES 6.13, 6.14, and 6.15, that the microstructure evolution has been captured accurately by the proposed approach. The equivalent strain and temperature history at the chosen locations (see FIGURE 


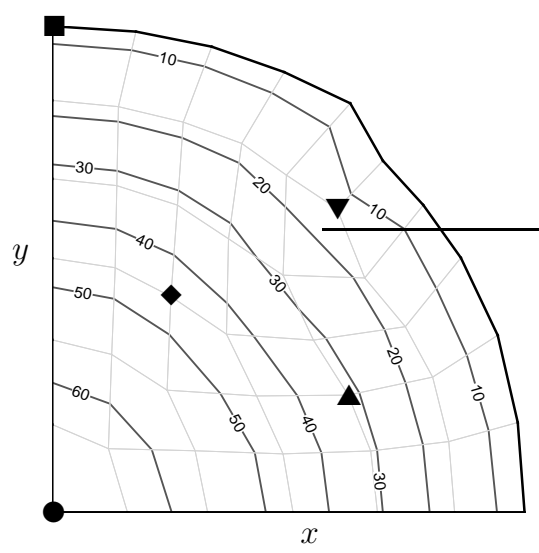

(a) Initial REX. grain size $\left(D_{r e x}\right)$.

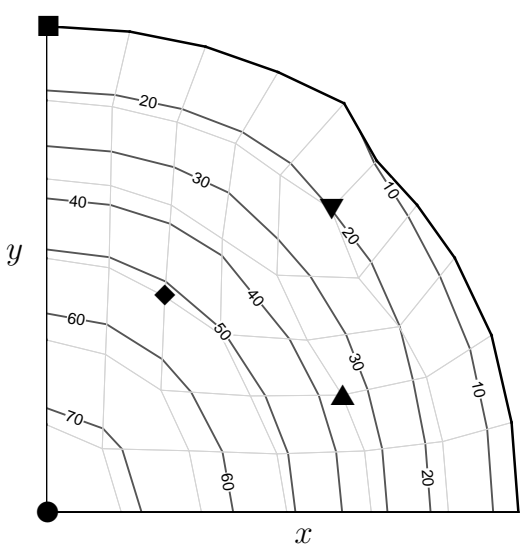

(b) Rec. REX grain size $\left(d_{\text {rexrex }}\right)$.

FIGURE 6.14: REX family grain size $(\mu m)$ after air cooling at the end of $4^{\text {th }}$ stand.

6.1(a)) are shown in FIGURES 6.17 and 6.16 respectively. In, FIGURE 6.17, the plot was created with actual strains and not-to-scale times as described for FIGURE 6.3. From these figures, it can be observed that the instantaneous equivalent strains reach values as high as 1.45 during the $13^{\text {th }}$ stand. Similarly, due to severe deformations imposed during the later stands, the temperatures also experience a significant increase. The initial stands experience a similar recrystallization phenomena observed in the 4-stand analysis. Therefore, the initial observation part is skipped in the 16-stand analysis. Streamline results are discussed in the next section.

\subsubsection{Streamline results}

The streamline results for the various benchmarking locations are depicted in FIGURES 6.18 through 6.22. The subfigures in all these plots are similar to the ones observed for a 4-stand analysis. However, the subfigure indicated by (c) depicts the microstructure evolution for times until the strained grain families are fully recrystallized. This enhances the analysis of the strained grain families closer. FIGURE 6.18 indicates the microstructure evolution for the central location in the billet indicated by symbol $\bullet$. The strained grain family vanishes at the end of the first interstand and the recrystallized family undergoes recrystallizations due to further deformations and 

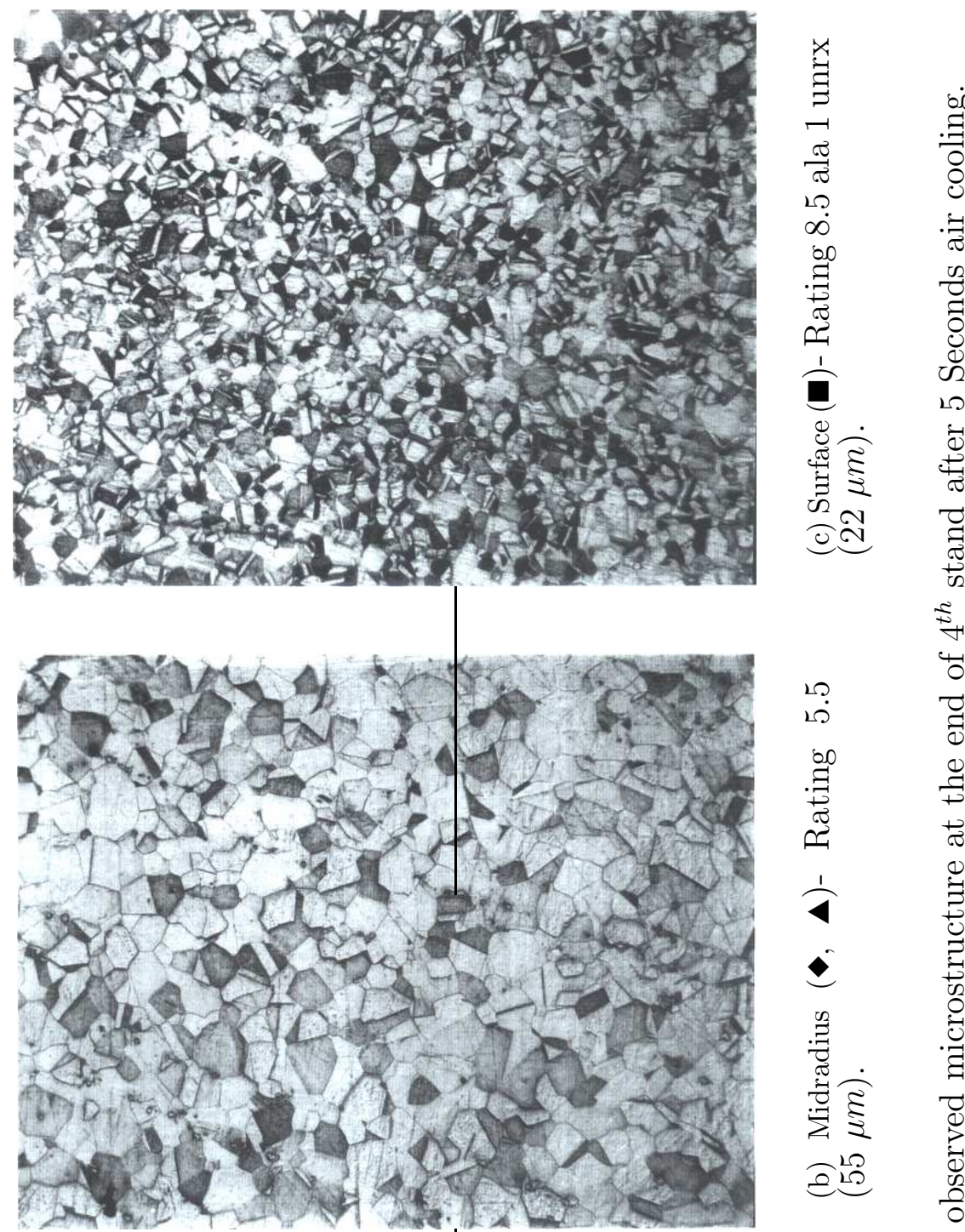

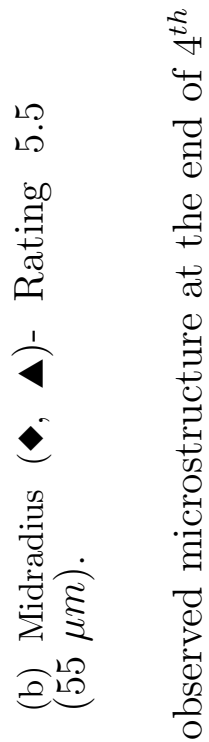

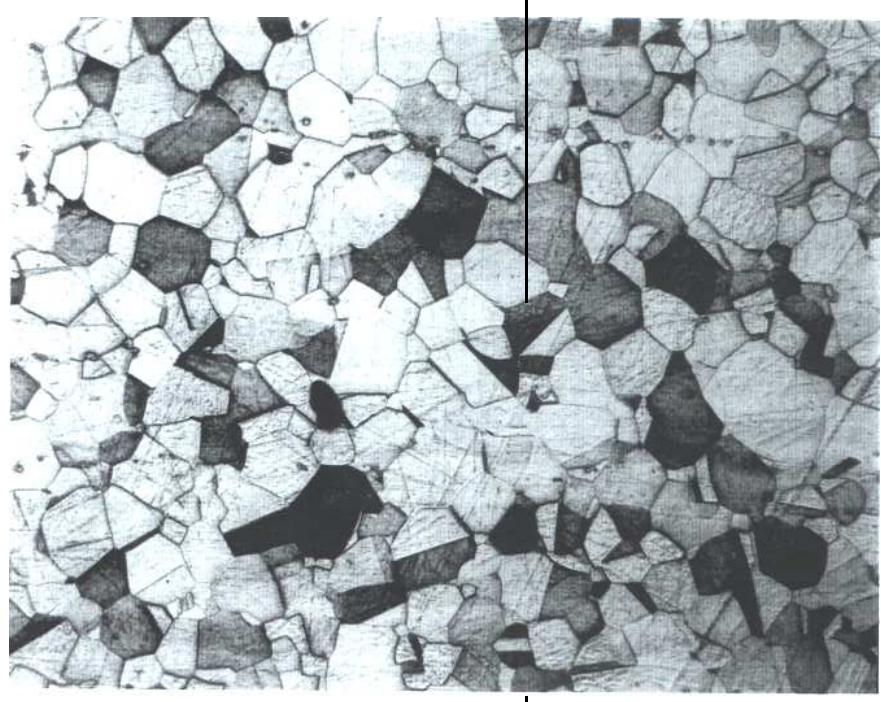

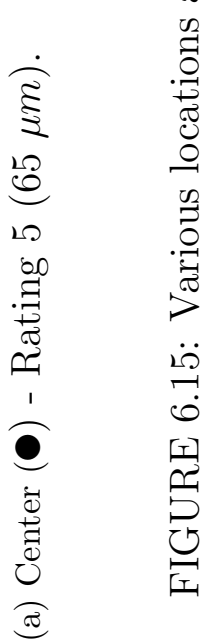




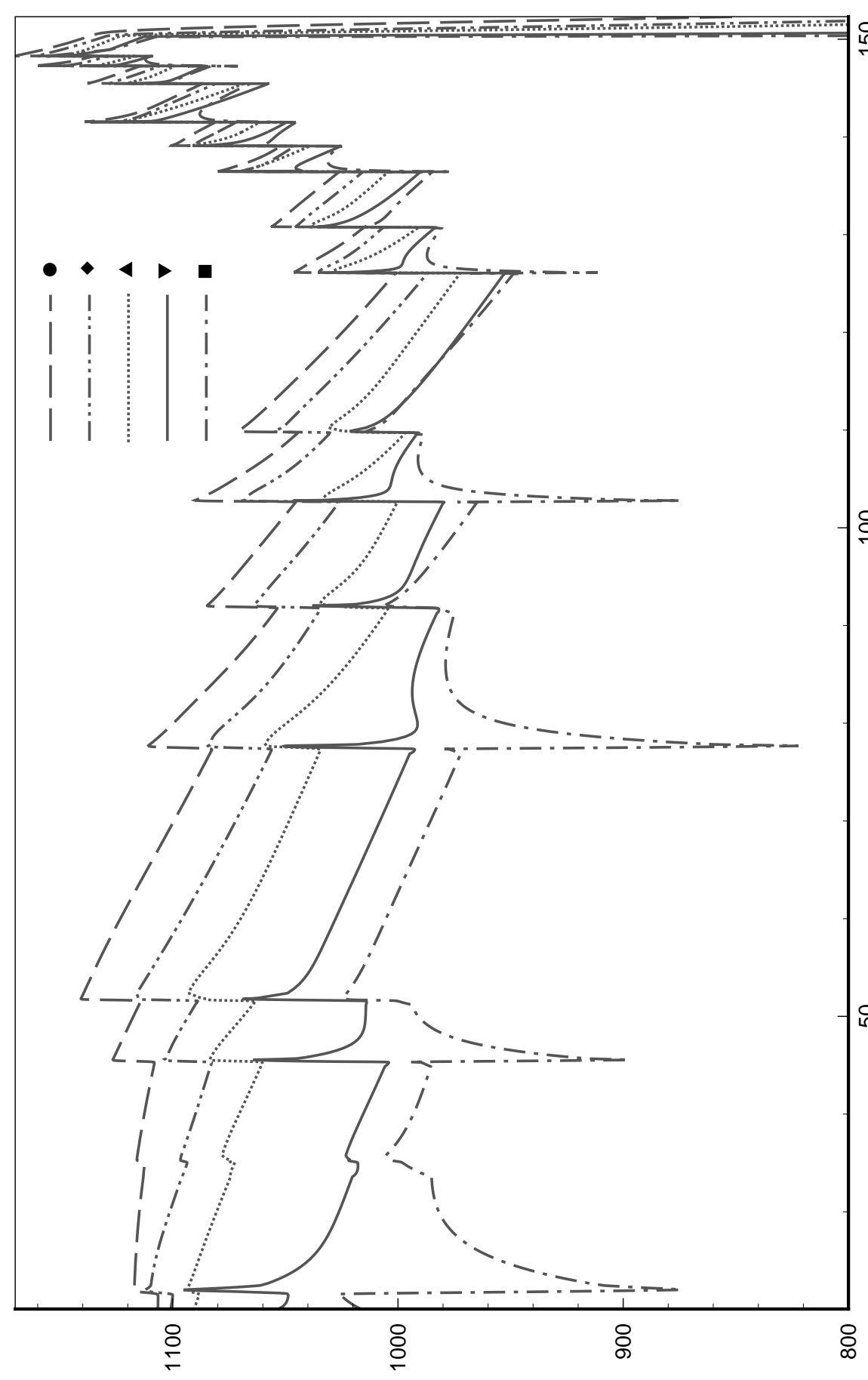

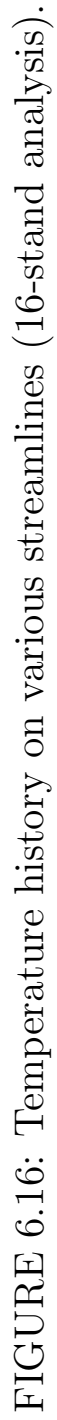

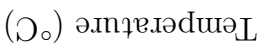


annealing during the rest of the stands. The recrystallization fraction $F_{\text {rex }}$ denoting the global recrystallization at this location reaches a value of 1.0 at the beginning of the deformation in the second stand. A closer look at the end of $10^{\text {th }}$ stand indicates an increasing trend of grain size characterized by $d_{\text {rexrex }}$. This phenomenon is observed in all the locations considered. Location indicated by the symbol $\bullet$ experiences a similar trend regarding the microstructural variables (see FIGURE 6.19). For the midradius location, a small remaining portion of the strained family undergoes full recrystallization at the end of the $2^{\text {nd }}$ interpass (see FIGURE 6.21).

An interesting phenomenon is observed in the case of the subsurface location (indicated by $\boldsymbol{\nabla}$ ) in FIGURE 6.21. An insignificant portion of strained grain family experiences full recrystallization during the $9^{\text {th }}$ interpass. However, this does not contribute much to the global microstructure characteristics. The surface location (see FIGURE 6.22), contains both strained and recrystallized grain families till the $6^{\text {th }}$ stand and then the strained grain family vanishes.

\subsubsection{Final observations}

It is clear from the bench marking location results that the microstructure at the end of $16^{\text {th }}$ stand contains only recrystallized grains. That is, grains are fully recrystallized. A contour plot of the recrystallized grain sizes characterized by the variables $D_{\text {rex }}$ and $d_{\text {rexrex }}$ are shown in FIGURE 6.23. The grain size distribution is almost uniform around $20 \mu \mathrm{m}$ (see FIGURE 6.23(a)) for the $D_{\text {rex }}$ which characterizes the overall grain size distribution while the recrystallized grains (characterized by $d_{\text {rexrex }}$ in FIGURE 6.23(b)) show slightly larger grains close to the center, since the center does not cool quickly. The actual microstructure observed at the end of cooling after 16-stand rolling of the considered material is shown in FIGURE 6.24. The calculated microstructure results are in excellent agreement with the observed microstructure.

\subsection{Discussions}

The generic microstructure algorithm is capable of accommodating various microstructural models developed empirically. In the current work, the parameters used are based on Reference [49] for DRX. For the MDRX, the recrystallization process parameters were considered from Reference [45], however, the grain size during MDRX 


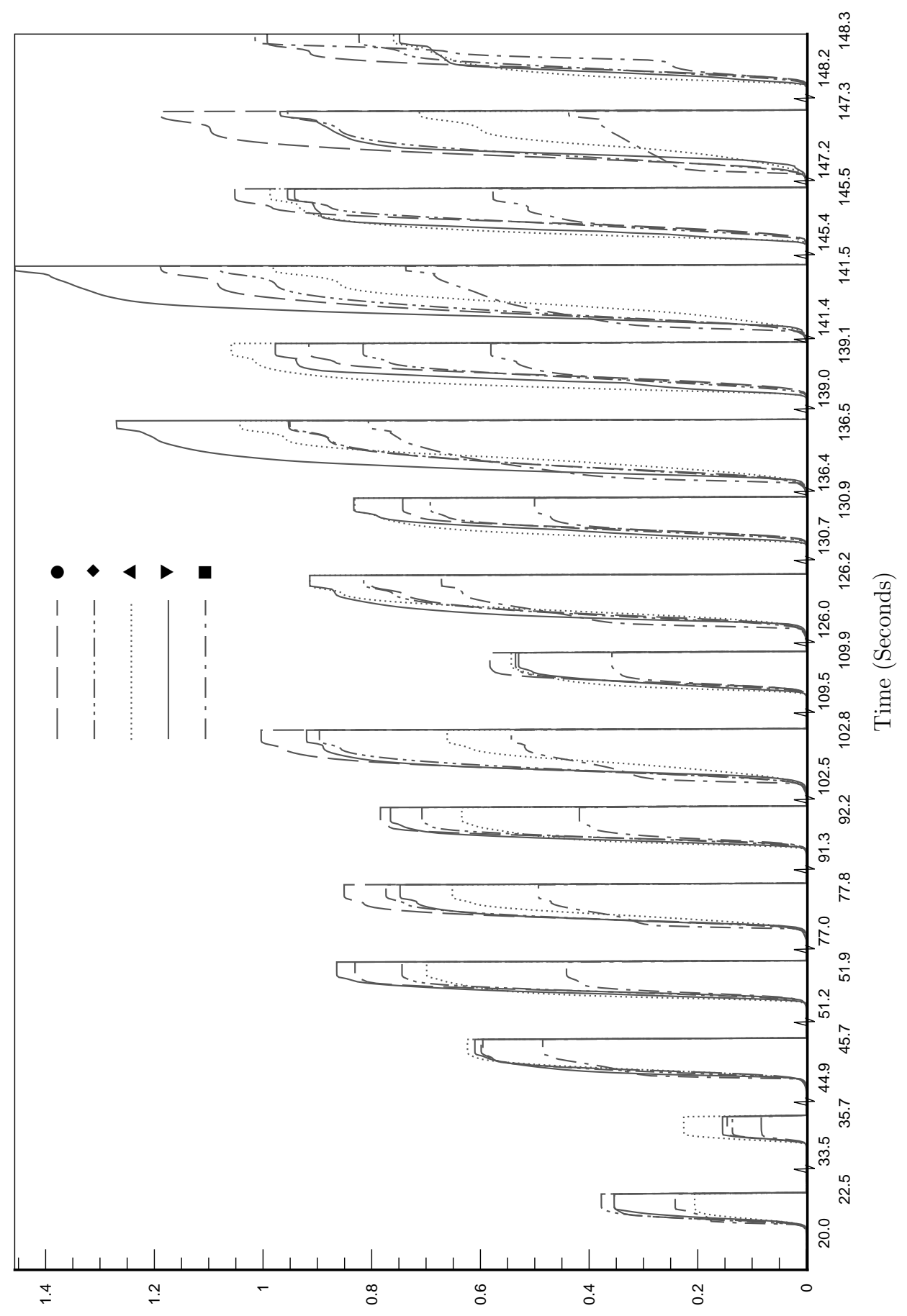

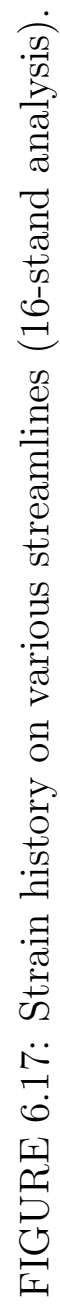

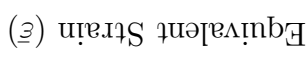




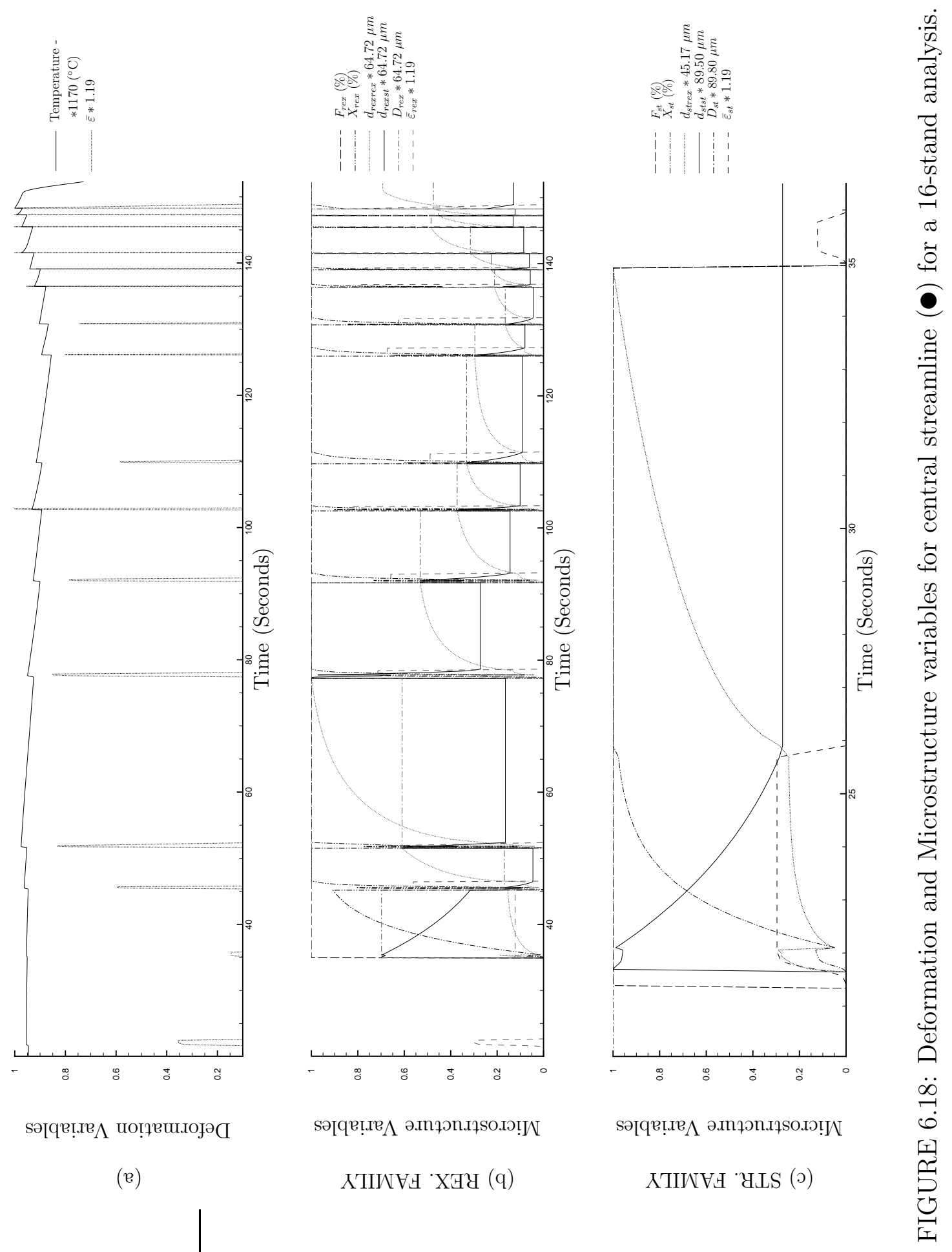




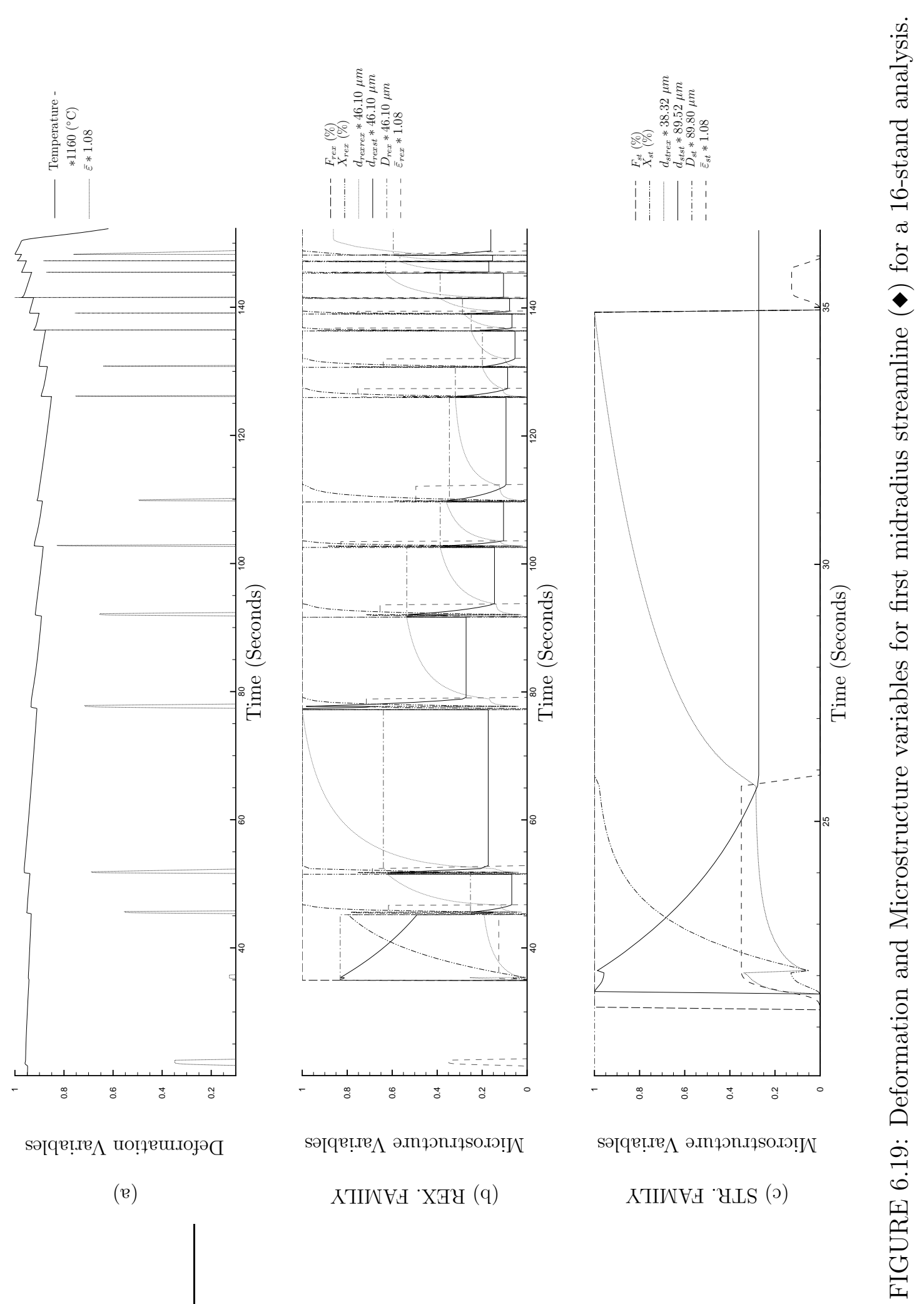




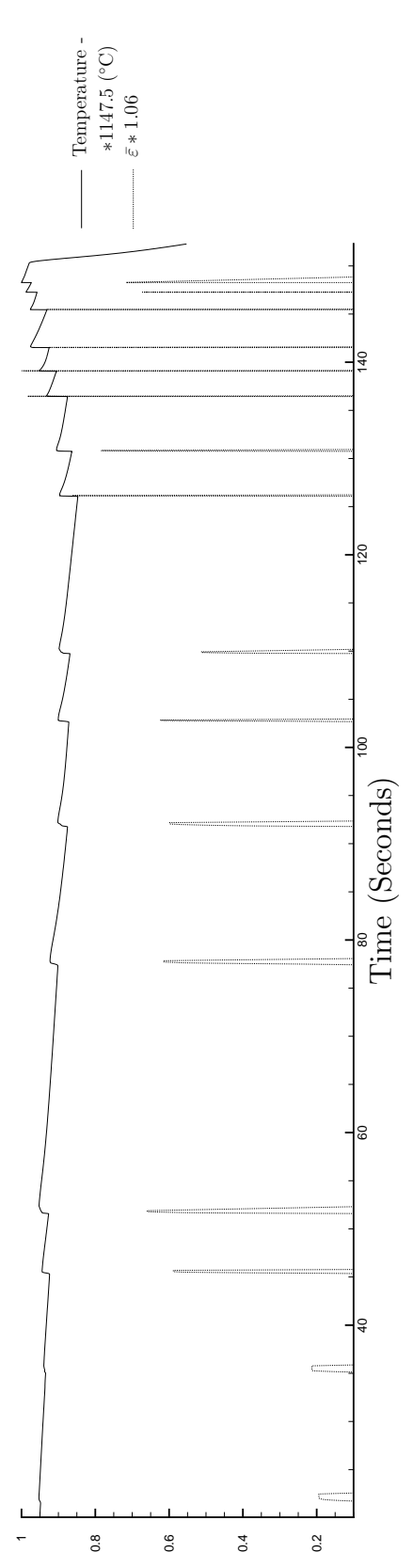

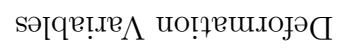

(e)
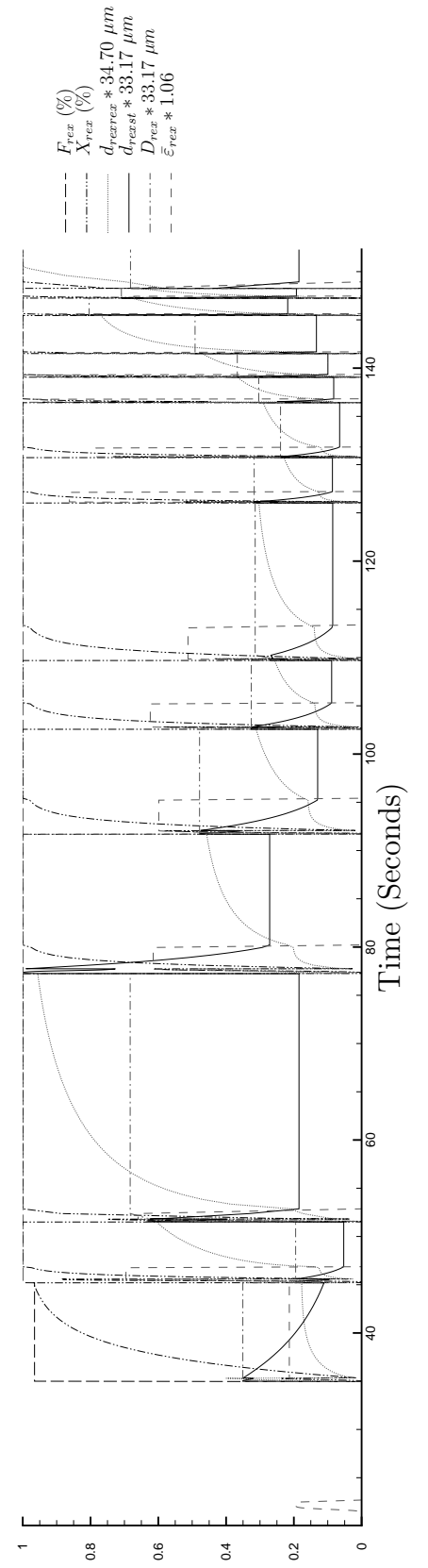

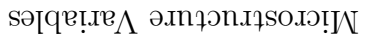

ХTINНH ХЯ̈Ч (q)

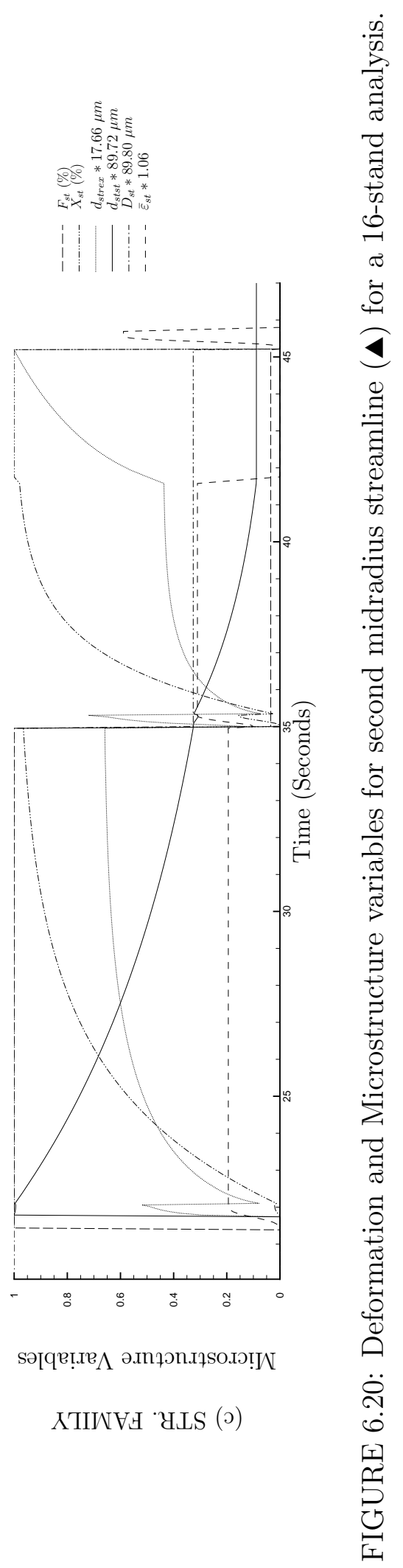




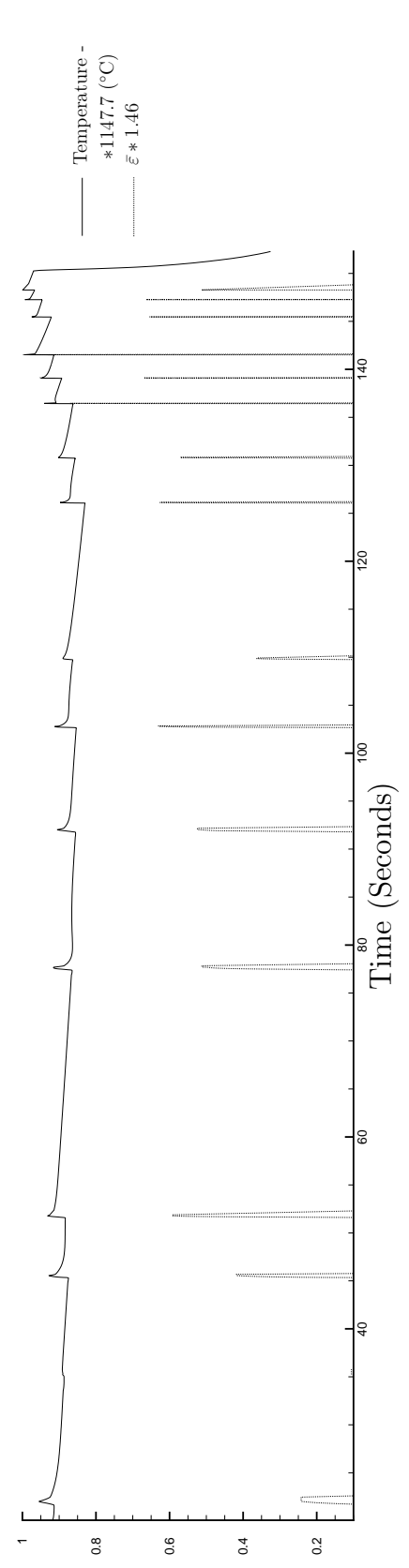

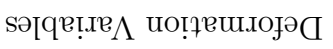

(e)
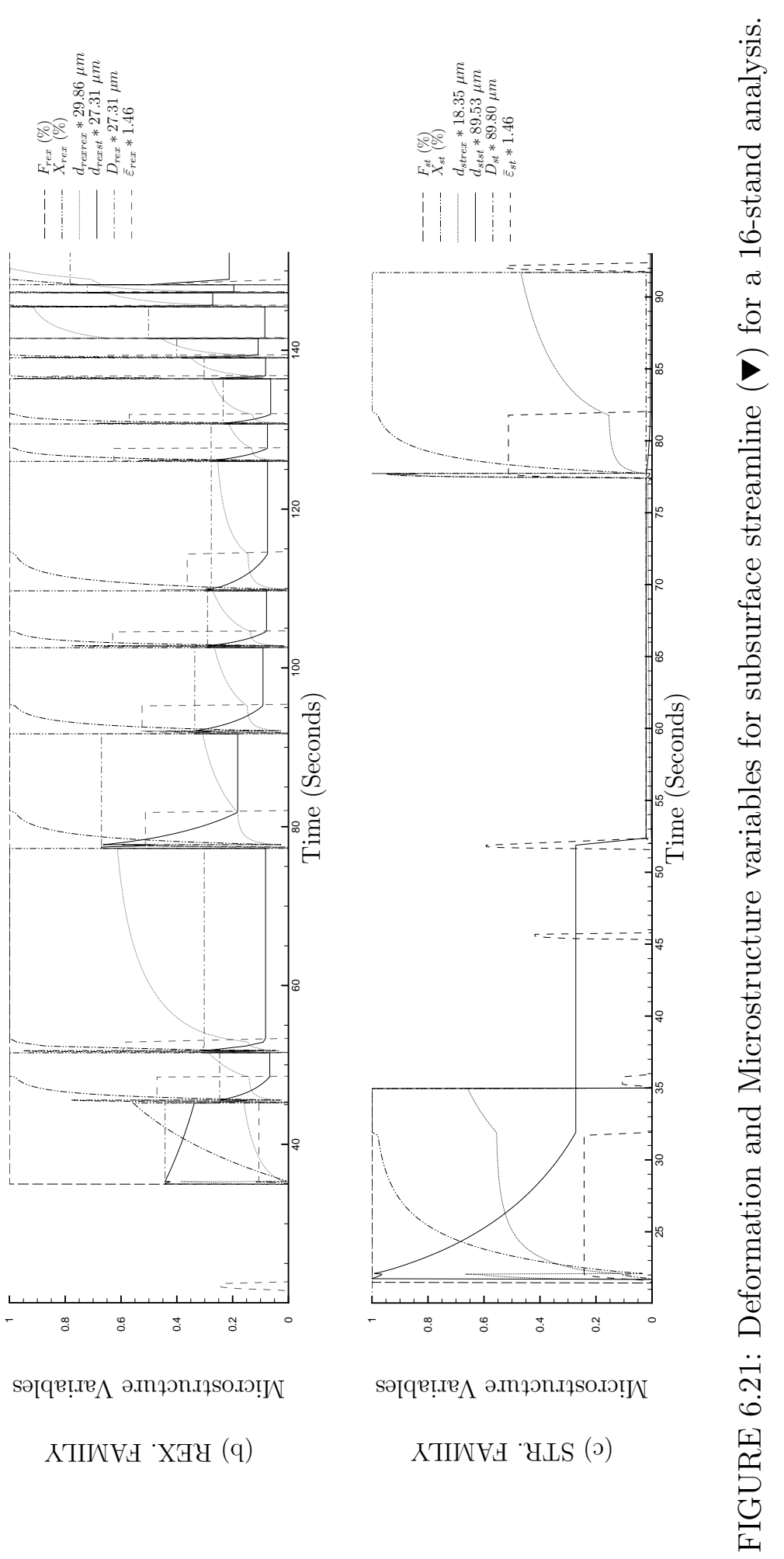


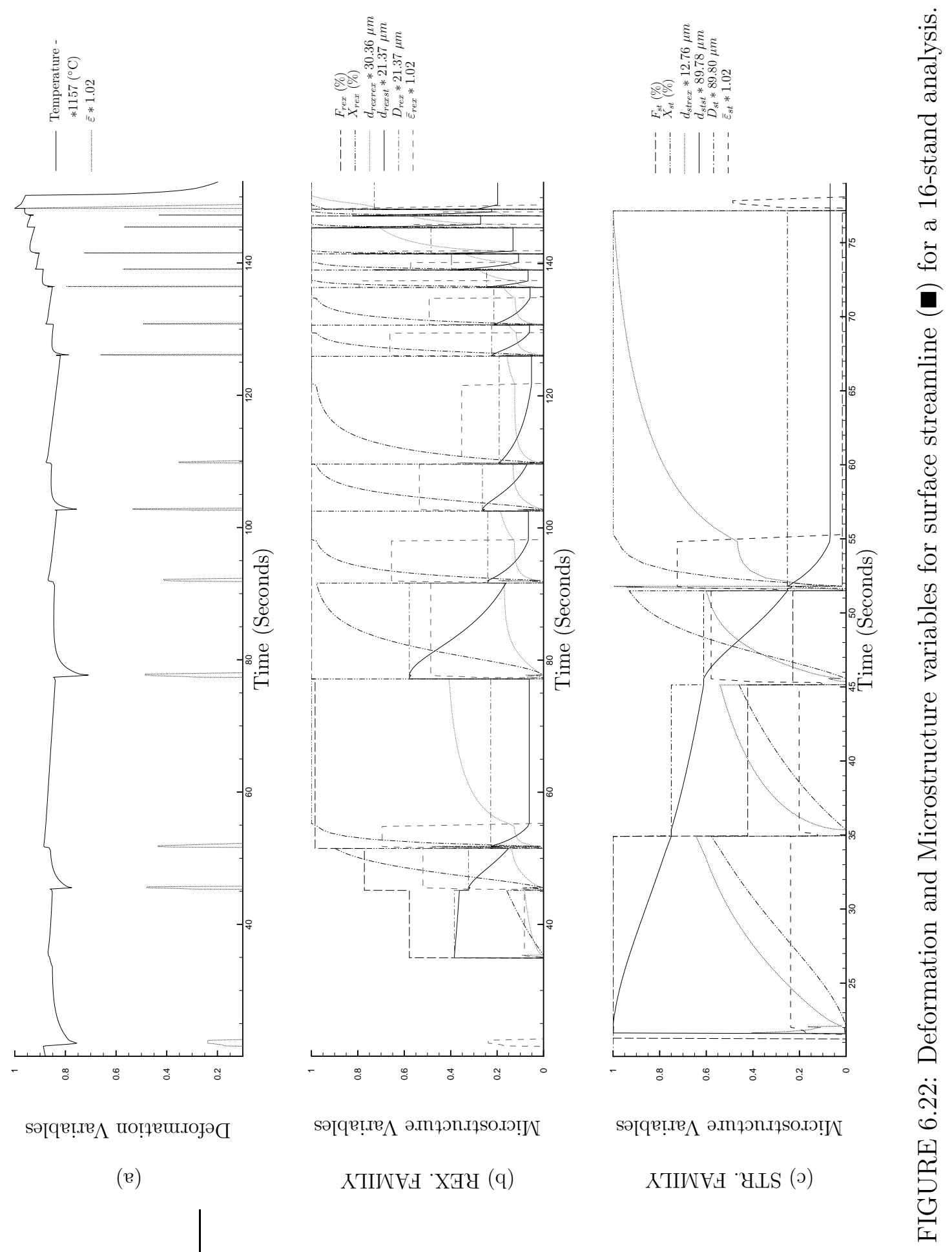




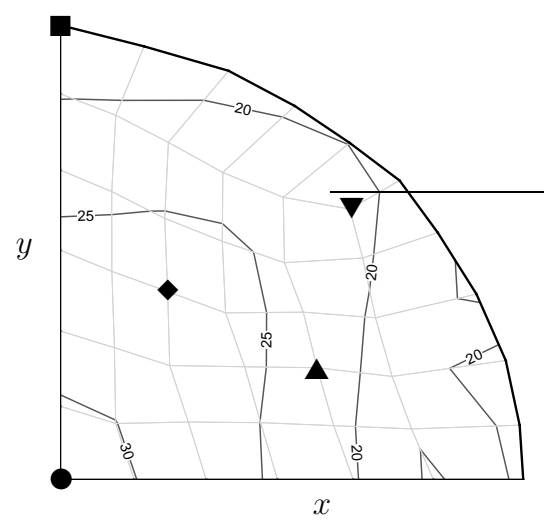

(a) Initial Rec. Grain Size $\left(D_{r e x}\right)$.

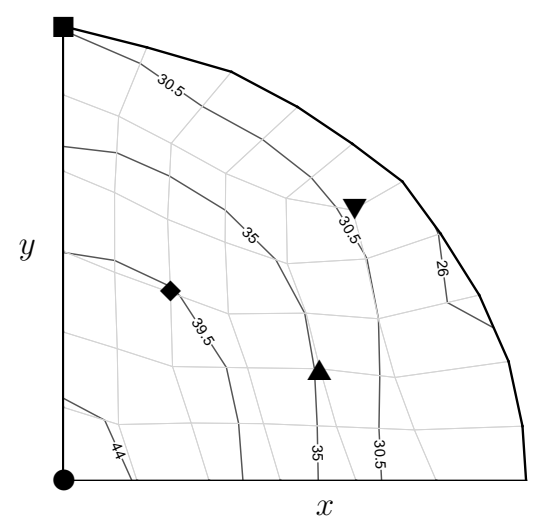

(b) Rec. Grain Size $\left(d_{\text {rexrex }}\right)$.

FIGURE 6.23: Grain sizes $(\mu m)$ at the end of cooling after $16^{\text {th }}$ stand.

was calculated based on Reference [62]. The static grain growth was calculated based on the cubic laws found in Reference [49], however, the first parameter in the equation 5.23 had to be modified for Alloy 718. This model is in very good agreement with the experimental observations for the previously mentioned 4-stand and 16-stand analyses.

When microstructural processes such as DRX occurs, the increase of strain and temperature favors dynamic softening. Grain size is a function of the deformation variables such as temperature, strain, and strain rate. Temperature enhances dynamic softening, and as observed during the later stands, where a significant increase in temperature is observed, the grain sizes tend to increase. Strain influences the microstructure significantly as observed by other authors $[56 ; 61 ; 65]$. The streamline figures indicate this conclusion. The fraction recrystallized due to DRX increases due to increase in the addition of strain and the accumulated strain at the end of deformation influences the MDRX since DRX does not completely recrystallize the grains in any case for Alloy 718 material. The increase in strain increases the dislocation density and nucleation of recrystallized grains as the deformation continues. An increased rate in MDRX after deformation at high strain rates as a result of adiabatic heating is explained in References [70]. Even though, a graphical representation of 


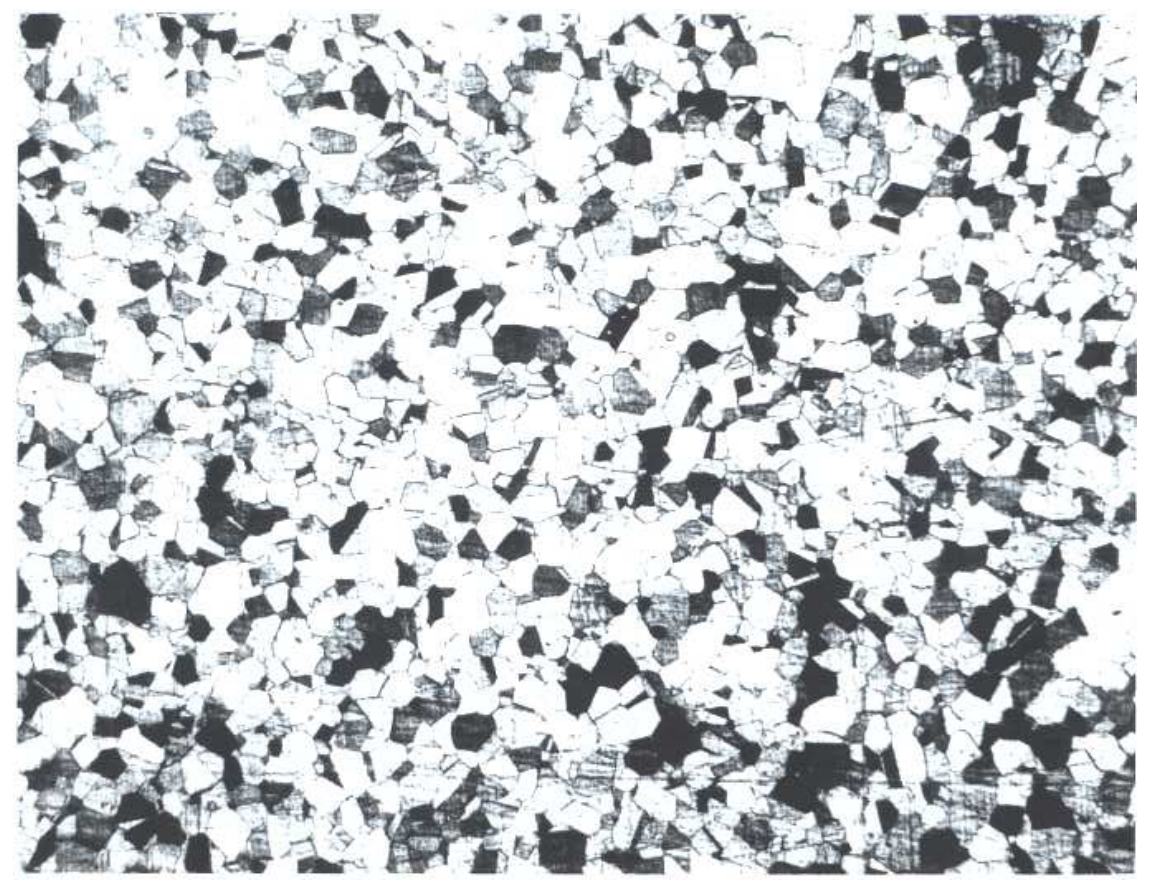

FIGURE 6.24: Uniform microstructure at any location at the end of $16^{\text {th }}$ stand (ASTM 8 or $22 \mu m$ uniform) (courtesy: ATI Allvac).

this effect is not included here, it has been generally observed that during the later stands, especially after $11^{\text {th }}$ stand the rate of MDRX has been observed to be significantly faster than during the other stands. The variation of grain size is correlative to the behavior of work hardening and dynamic softening existing in Alloy 718 during

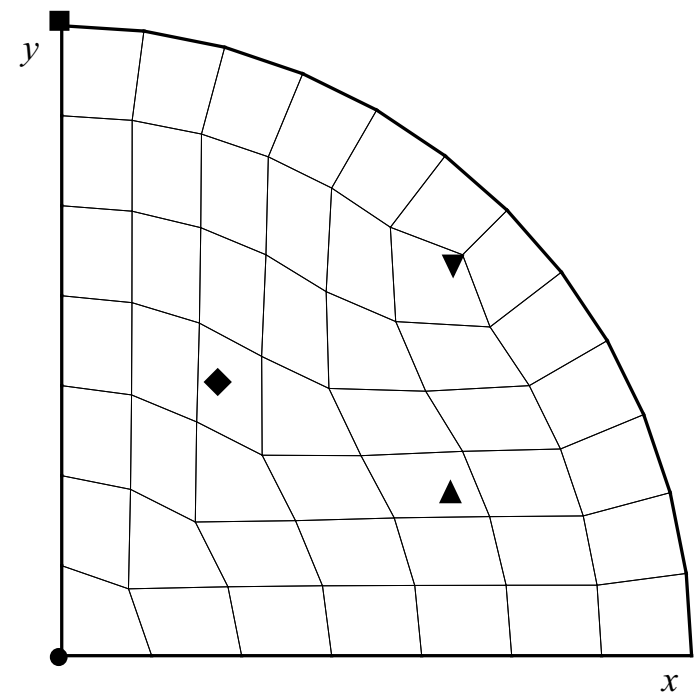

FIGURE 6.25: Finer mesh considered for microstructure comparison. 


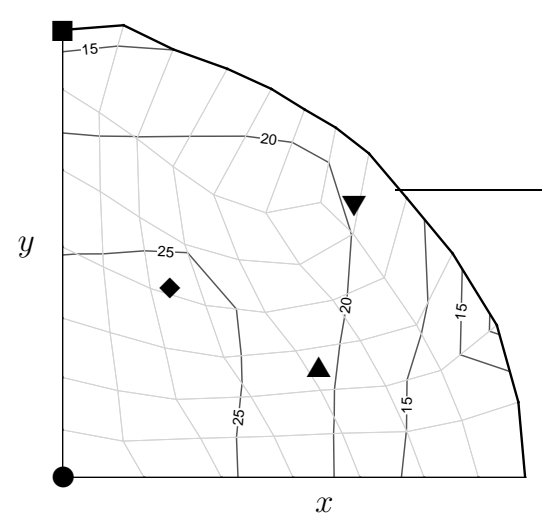

(a) Initial Rec. Grain Size $\left(D_{r e x}\right)$.

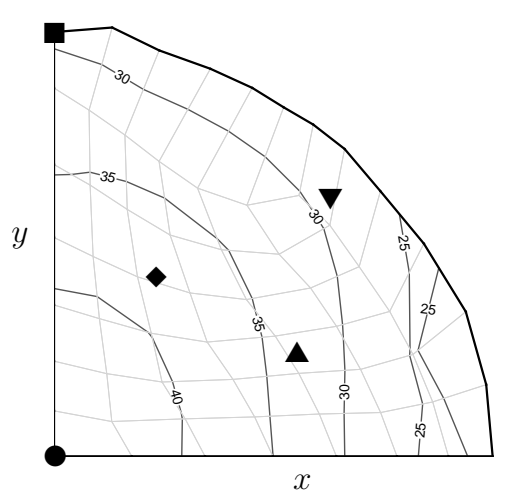

(b) Rec. Grain Size $\left(d_{\text {rexrex }}\right)$.

FIGURE 6.26: Grain sizes $(\mu m)$ at the end of cooling after $16^{\text {th }}$ stand for finer mesh.

hot deformation.

When the temperature increases, dynamic softening occurs and the grain size increases. When the strain rate increases, work hardening occurs and the grain size decreases. An equilibrium is reached between the work hardening (due to the increase in strain) and dynamic softening (due to temperature increase). Increasing the holding time increases the volume fraction recrystallized. Long holding times after deformation allows complete recrystallization of microstructure [61]. This is witnessed during the interpasses, where the MDRX is a significant contributor.

\subsection{Finer mesh results}

In this section, a finer mesh is considered to study the effect of FE mesh on the microstructure prediction. A mesh involving six core divisions and one outer division, shortly, a 6x1 mesh as shown in FIGURE 6.25. The finer mesh contains an additional 13 elements and 15 nodes in comparison to the standard mesh (5 core divisions and 1 outer division) considered (see FIGURE 6.1(a) for the whole analysis discussed in this Chapter. FIGURE6.27 shows the comparison of one of the deformation variables, the temperature and shape for both the meshes at the end of cooling analysis after the $16^{\text {th }}$ stand. It can be observed that the temperatures compare very close to each other. The predicted shapes are identical in dimensions except at the top of the 
rolled bar where the finer mesh determined a flat geometry and the standard mesh predicted a sharper geometry. However, the difference in the predicted dimensions is negligible. In addition to the closeness of predicted temperatures between the standard and finer meshes, it was observed that the other deformation variables also showed similar patterns.

The results from the microstructure analysis are presented in FIGURE 6.26. From FIGURE 6.26(a) and FIGURE 6.23(a), it is clear that the recrystallized grain sizes at the beginning of the $16^{\text {th }}$ stand are very closely comparable. In addition, from FIGURE 6.26(b) and FIGURE 6.23(b), it can be observed that the average recrystallized grain sizes are very close to each other. From this comparison, it is concluded that a finer mesh which requires additional computational time and resources is not necessary for the approach developed and presented in the previous Chapter.

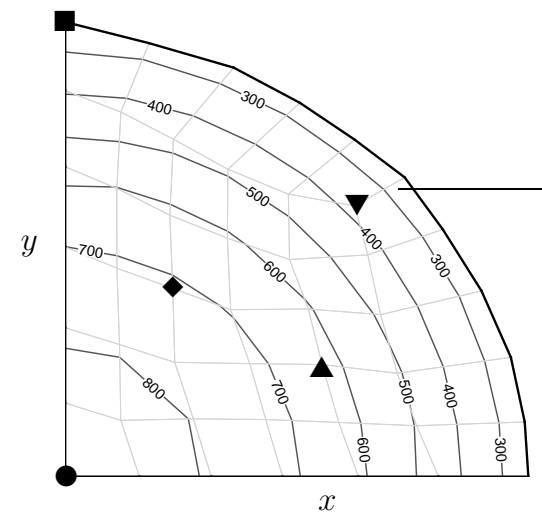

(a) Temperature contours for $5 \mathrm{x} 1$ mesh.

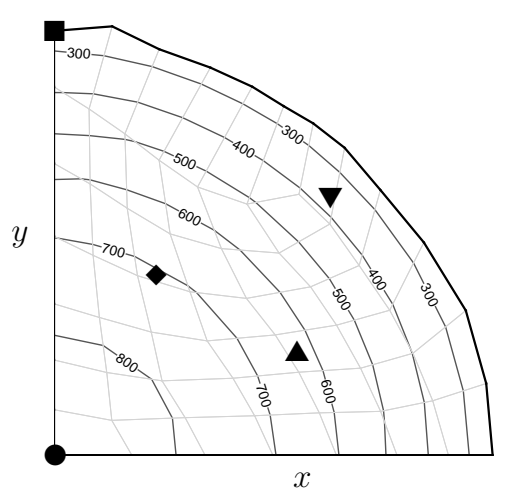

(b) Temperature contours for $6 \times 1$ mesh.

FIGURE 6.27: Temperature comparison for different meshes.

\subsection{Conclusion}

The current procedure incorporates the approach by which a total recrystallization fraction is calculated by taking into account the residual strain and also incorporates a total recrystallized fraction that incorporates fractions of previously recrystallized grain families as described in References $[71 ; 61]$. The developed procedure assumes 
that the grain matrix does not contain excessive $\delta$ phase [61]. However, $\delta$ phase disappears at temperatures above $1000{ }^{\circ} \mathrm{C}$ [46], close to the solvus temperature of $\approx$ $1010{ }^{\circ} \mathrm{C}[49]$.

In the current procedure the observed temperatures are mostly above the solvus region and the averaging procedure is considered applicable in predicting the microstructure dominated by $\gamma$ matrix. In the current work, the microstructure is considered isotropic and equiaxed $[61 ; 65]$. A geometric modeling approach discussed elsewhere [61] can accommodate the variances in the geometry of the microstructure. 


\section{CHAPTER 7: CONCLUSIONS \& FUTURE WORK}

\subsection{Summary of the present work}

As mentioned in Chapter 1, nickel-base superalloys possess high flow stresses and can be hot worked only in a small temperature range as evident from the streamline figures explained in Chapter 6. If the hot working temperature exceeds the maximum in the range, detrimental grain growth may be initiated. On the other hand, if the temperatures are too low, undesired phases might precipitate [70]. These facts need to be taken into consideration when devising a hot working strategy. Since the final microstructure controls the product properties which are in fact, controlled by the deformation variables, it is important to know the evolution of the microstructure quickly and accurately. Empirical laws attempt to capture the behavior of a particular alloy under laboratory conditions and are very limited [80] in their prediction capabilities. However, they are simple in nature and when applied in predicting the average microstructure, they are very efficient and excellent tools in designing a multi-stand rolling sequence that was the focus of this work. The prediction of microstructure by employing an appropriate model available in the literature still requires a scientific approach that captures the evolution of the microstructure. Specifically, such a procedure is not currently available for the multi-pass rolling of any superalloy. The current work encompassed the development of such a procedure and its validation in the context of Alloy 718.

\subsection{Conclusions}

In line with the set objectives listed in Chapter 1, the accomplishments are listed as follows:

- A detailed mesh re-zoning algorithm was developed and validated in the context of a 16-stand multi-pass rolling of the alloy material considered for the current work. 
- The FE package, RAWHIDE, was modified to allow printing of the temperature history during the interpass heat transfer analyses, since the accurate information of this history is important in predicting the microstructure accurately.

- A microstructural modeling algorithm was developed for predicting the microstructure for a multi-stand rolling process based on flow formulation.

- The developed algorithm was validated for Alloy 718 for a 4-stand rolling analysis involving air cooling at the end of the $4^{\text {th }}$ stand and for a 16-stand rolling analysis involving air and water cooling at the end of the $16^{\text {th }}$ stand.

The predicted numerical results are in excellent agreement with the observed microstructure by incorporating suitable empirical models found in literature [45; 49; 62] for various microstructural processes.

\subsection{Future Work}

The developed microstructure algorithm was applied for a specific proprietary rolling schedule and for a specific material only. However, there are many superalloys that can be explored with the developed procedure. In addition, other rolling schedules can also be explored. Even though, the present algorithm is tested on a rolling process which was modeled using flow formulation, the algorithm is capable of predicting microstructure even for other formulations found in many commercial FE packages. 


\section{BIBLIOGRAPHY}

[1] Numerical Simulation of a Multi-Stand Rolling Mill, R. S. Minisandram, E. G. Thompson, R. M. Forbes Jones, and R. Stedje-Larsen, Simulation of Materials Processing: Theory, Methods and Applications - Proceedings of the $7^{\text {th }}$ International Conference on Numerical Methods in Industrial Forming ProcessesNUMIFORM 2001, Toyohashi, Japan, June 2001, pp. 549-554, A. A. Balkema (2001).

[2] Model for radial forging of superalloys, R. S. Minisandram, Advanced materials \& Processes, Vol. 148 (4), pp. 47-49, ASM International (1995).

[3] Numerical study of the effects of die profile on extrusion, R. E. Smelser, O. Richmond, \& E. G. Thomson, Proceedings of the International Conference on Numerical Methods in Industrial Forming Processes-NUMIFORM 1986, Gothenburg, Sweden, August 1986, pp. 305-312, A. A. Balkema (1986).

[4] Iterative method for constrained and mixed approximation. An inexpensive improvement of FEM performance, O. Zienkiewicz, J. Vilotte, S. Toyoshima, and S. Nakazawa, Computational Methods in Applied Mechanics and Engineering, Vol. 51 (1-2), pp. 3-29, Elsevier Science (1985).

[5] Flow formulation for numerical solution of forming processes, O. Zienkiewicz, Numerical Analysis of Forming Processes, pp. 1-69, Wiley (1984).

[6] Average and complete incompressibility in the finite element method, E. G. Thompson, International Journal for Numerical Methods in Engineering, Vol. 9, pp. 925-932, Wiley (1975).

[7] Numerical analysis of a new Eulerian-Lagrangian finite element method applied to steady-state hot rolling processes, J. Synka, et al., International Journal for Numerical Methods in Engineering, Vol. 62 (5), pp. 616-638, Wiley (2005).

[8] Elasticity equations for incompressible and nearly incompressible materials by a variational theorem, L. R. Hermann, AIAA Journal, Vol. 3 (10), pp. 1896-1900, AIAA (1965).

[9] Steady state analysis of elasto viscoplastic flow during rolling, E. G. Thompson and H. M. Berman, Numerical Methods in Industrial Forming Processes, Vol. 1, pp. 29-37, Pineridge (1982).

[10] Bulge predictions in steady state bar rolling processes, S. H. Lee, et al., International Journal for Numerical Methods in Engineering, Vol. 30, pp. 1403-1413, Wiley (1990).

[11] Some integration techniques for the analysis of viscoelastic flows, E. G. Thompson, et al., International Journal for Numerical Methods in Fluids, Vol. 3, pp. 165-177, Wiley (1983). 
[12] Hydrodynamics, H. Lamb, Dover (1932).

[13] Finite element thermomechanical models for metal forming, P. R. Dawson, Ph.D. Dissertation, Colorado State University, Fort Collins (1976).

[14] Finite element thermo-mechanical model for creeping convection, A. Sato, Ph.D. Dissertation, Colorado State University, Fort Collins (1975).

[15] Coupled thermo-mechanical-metallurgical analysis during the cooling process of steel pieces, A. M. Habraken, M. Bourdouxhe, European Journal of Mechanics A/Solids, vol. 11 (3), pp. 381-402, Elsevier (1992).

[16] A practical guide to splines, C. de Boor, Applied Mathematical Sciences, Vol. 27, Springer (1978).

[17] An interpolation curve using a spline in tension, D. G. Schweikert, Journal of Mathematics and Physics, Vol. 45 (3), pp. 312-317, Brown University (1965).

[18] Scalar- and planar- valued curve fitting using splines under tension, A. K. Cline, Numerical Mathematics: Communications of the ACM, Vol. 17 (4), pp. 218-220, ACM (1974).

[19] A method for computing the tension parameters in convexity-preserving splinein-tension interpolation, N. S. Sapidis, P. D. Kaklis, and T. A. Loukakis, Numerische Mathematik, Vol. 54 (2), pp. 179-192, Springer (1989).

[20] Automatic tension adjustment for interpolatory splines, Y. Fletcher, D. F. McAllister, Computer graphics \& applications, Vol. 10, pp. 10-17, IEEE (1990).

[21] A mesh re-zoning technique for finite element simulations of metal forming processes, J. H. Cheng, and N. Kikuchi, International Journal for Numerical Methods in Engineering, Vol. 23 (2), pp. 219-228, Wiley (1986).

[22] Automatic adaptive remeshing for finite element simulation of forming processes, J. H. Cheng, International Journal for Numerical Methods in Engineering, Vol. 26 (1), pp. 1-18, Wiley (1988).

[23] An automatic remeshing technique for finite element simulation of forming processes, A. M. Habraken, and S. Cescotto, International Journal for Numerical Methods in Engineering, Vol. 30 (8), pp. 1503-1525, Wiley (1990).

[24] Mesh re-zoning of 2D isoparametric elements by inversion, R. H. Crawford, et al., International Journal for Numerical Methods in Engineering, Vol. 28 (3), pp. 523-531, Wiley (1989).

[25] Prof. W. R. Franklin's homepage (http://www.ecse.rpi.edu/ Homepages/wrf/Research/Short_Notes/pnpoly.html).

[26] Point in Polygon Strategies, E. Haines, Graphics Gems IV, ed., Paul Heckbert, pp. 24-46, Academic Press, (1994). 
[27] Modeling microstructure and its effects during multipass hot rolling, J. H. Beynon, C. M. Sellars, ISIJ International, Vol. 32 (3), pp. 359-367, ISIJ (1992).

[28] Recrystallization and grain growth in hot rolling, C. M. Sellars and J. A. Whiteman, Metal science, pp. 187-194, Metals Society (1979).

[29] Modeling microstructural development during hot rolling, C. M. Sellars, Materials Science and Technology, Vol. 6 (11), pp. 1072-1081, Maney Publishing (1990).

[30] Recrystallization of metals during hot deformation, C. M. Sellars, Philosophical Transactions of the Royal Society of London. Series A, Mathematical and Physical Sciences, Vol. 288 (1350), pp. 147-158, The Royal Society (1978).

[31] Development of constitutive equations for modelling of hot rolling, S. B. Davenport, N. J. Silk, C. N. Sparks and C. M. Sellars, Materials science and Technology, Vol. 16 (5), pp. 539-546, Maney Publishing (2000).

[32] Microstructure evolution during rod and bar rolling, P. D. Hodgson, R. E. Gloss, G. L. Dunlop, 32nd Mechanical Working and Steel Processing Conference Proceedings, Cincinnati, Ohio, October 1990, Vol. XXVIII, pp. 527-538, Iron \& Steel Society (1990).

[33] Development of dislocation-based unified material model for simulating microstructure evolution in multiplass hot rolling, J. Lin, et al., Philosophical Magazine, Vol. 85 (18), pp. 1967-1987, Taylor \& Francis (2005).

[34] Prediction of microstructural changes during hot rod rolling, S. Serajzadeh, International Journal of Machine Tools \& Manufacture, Vol. 43 (14), pp. 1487-1495, Elsevier (2003).

[35] Thermomechanical modeling of hot slab rolling, S. Serajzadeh, Materials science and technology, Vol. 21 (1), pp. 93-102, Maney Publishing (2005).

[36] Prediction of dynamic recrystallization kinetics during hot rolling, S. Serajzadeh, Modelling and Simulation in Materials Science and Engineering, Vol. 12 (6), pp. 1185-1200, Institute of Physics Publishing (2004).

[37] Mathematical and physical simulation of the properties of hot rolled products, J. G. Lenard, M. Pietrzyk, L. Cser, Elsevier (1999).

[38] Development and validation of a finite element model for hot rolling using ABAQUS/STANDARD, A. Mukhopadhyay, I. C. Howard and C. M. Sellars, Materials Science and Technology, Vol. 20 (9), pp. 1123-1133, Maney Publishing (2004).

[39] Numerical simulation method for designing thermomechanical treatments, illustrated by bar rolling, K. Karhausen, R. Kopp, M. M. de Souza, Scandinavian Journal of Metallury, Vol. 20 (6), pp. 351-363, Wiley (1991). 
[40] Model for integrated process and microstructure simulation in hot forming, K. Karhausen, R. Kopp, Steel Research International, Vol. 63 (6), pp. 247-256, Verlag Stahleisen (1992).

[41] Microstructure and mechanics interaction in the modeling of hot rolling of rods, P. Pauskar, R. Shivpuri, CIRP Annals - Manufacturing Technology, Vol. 48 (1), pp. 101-104, Elsevier (1999).

[42] Prediction of microstructural evolution in hot rolling, F. Wang et al., Journal of Material Processing Technology, Vol. 177 (1-3), pp. 530-533, Elsevier (2006).

[43] Thermo-mechanical modeling of two phase rolling and microstructural evolution in the hot strip mill Part II. Microstructure evolution, M. D. Phaniraj et al., Journal of Material Processing Technology, Vol. 178 (1-3), pp. 388-394, Elsevier (2006).

[44] The structural evolution of superalloy ingots during hot working, R. M. Forbes, L. A. Jackman, JOM, 51 (1), pp. 27-31, TMS (1999).

[45] Computer simulation of microstructure evolution during hot forging of waspaloy and nickel alloy 718, D. Huang, et al., Microstructure Modeling and Prediction During Thermomechanical Processing, ed. R. Srinivasan et al., Warrendale, PA, pp. 137-147, TMS (2001).

[46] Effects of dynamic and metadynamic recrystallization on microstructures of wrought IN-718 due to hot deformation, L. X. Zhou, T. N. Baker, Materials Science and Engineering A, Vol. 196 (1-2), pp. 89-95, Elsevier (1995).

[47] Finite-element modeling of forging of nickel based superalloys, J. Kusiak, et al., 41st Mechanical Working and Steel Processing Conference Proceedings, Baltimore, Maryland, October, 1999. Vol. XXXVII, pp. 683-688, Iron \& Steel Society (1990).

[48] Deformation and recrystallization behavior during hot working of a coarse-grain, nickel-base superalloy ingot material, S. L. Semiatin, et al., Metallurgical and materials transactions A, Vol. 35 (2), pp. 679-693, Springer (2004).

[49] Modeling microstructural development in the forging of waspaloy turbine engine disks, G. Shen, Ph.D. Dissertation, Ohio State University (1994).

[50] Microstructure modeling in superalloy forging, G. Shen, Cold and Hot Forging: Fundamentals, ed. T. Altan, et al., pp. 247-255, ASM International (2005).

[51] Modeling microstructural development during the forging of waspaloy, G. Shen, S. L. Semiatin, R. Shivpuri, Metallurgical and materials transactions A, Vol. 26 (7), pp. 1795-1803, Springer (1995).

[52] Designing hot working processes of nickel-based superalloys using finite element simulation, R. Kopp, et al., Journal of Engineering for Gas Turbines and Power, Vol. 124 (4), pp. 931-935, ASME (2002). 
[53] Deformation behavior of waspaloy at hot-working temperatures, S. L. Semiatin, et al., Scripta materialia, Vol. 50 (5), pp. 625-629, Elsevier (2004).

[54] Microstructural model of gatorized waspaloy in the isothermal forging process, J. P. Hu, et al., Acta Metallurgica Sinica (English Letters), Vol. 14 (3), pp. 205-211, Elsevier (2001).

[55] Constitutive equations for cyclic plasticity of waspaloy, A. Abdul Latif, International Journal of Plasticity, Vol. 12 (8), pp. 967-985, Elsevier (1996).

[56] The dynamic and metadynamic recrystallisation of the IN 718, R. P. Guest, S. Tin, Proceedings of the 6th International Symposium on Superalloys 718, 625, 706 and Various Derivatives, ed. E. A. Loria, Pittsburgh, Pennsylvania, October, 2005, pp. 373-383, TMS (2005).

[57] Modelling microstructural transformations of nickel base superalloy IN 718 during hot deformation, R. P. Guest, S. Tin, Proceedings of the 6th International Symposium on Superalloys 718, 625, 706 and Various Derivatives, ed. E. A. Loria, Pittsburgh, Pennsylvania, October, 2005, pp. 385-397, TMS (2005).

[58] Mechanisms of grain refinement and superplastic behavior of alloy 718, B. P. Bewlay, et al., Microstructure modeling and prediction during thermomechanical processing, ed. R. Srinivasan, et al., pp. 175-185, TMS (2001).

[59] The influence of cold rolling on the precipitation of delta phase in INCONEL 718 alloy, W. C. Liu, et al., Scripta Materialia, Vol. 37 (1), pp. 53-57, Elsevier (1997).

[60] Representing the superplasticity of Inconel 718, B. Zhang, et al., Journal of Materials Processing Technology, Vol. 153-154, pp. 694-698, Elsevier (2004).

[61] EBSD Investigation and modelling of the microstructural evolutions of superalloy 718 during hot deformation, J. P. Thomas et al., Superalloys 2004, ed. K. A. Green, et al., pp. 959-968, TMS (2004).

[62] Finite-element analysis of microstructure evolution in the cogging of an alloy 718 ingot, J. T. Yeom et al., Materials Science and Engineering A, Vol. 449-451, pp. 722-726, Elsevier (2007).

[63] High temperature deformation behaviour of cast alloy 718, D. Zhao, et al., Proceedings of the 4th International Symposium on Superalloys 718, 625, 706 and Various Derivatives, ed. E. A. Loria, Pittsburgh, Pennsylvania, June, 1997, pp. 192-204, TMS (1997).

[64] Three-dimensional computer simulation of alloy 718: Ingot breakdown by cogging, D. Zhao, et al., Proceedings of the 4th International Symposium on Superalloys 718, 625, 706 and Various Derivatives, ed. E. A. Loria, Pittsburgh, Pennsylvania, June, 1997, pp. 163-172, TMS (1997). 
[65] Mathematical modeling of the hot deformation behaviour of Superalloy IN718, J. M. Zhang et al., Metallurgical and Material Transactions A, Vol. 30 (10), pp. 2701-2712, Springer (1999).

[66] Hot deformation behaviour of superalloy 718, C. I. Garcia et al., International Symposium on Superalloys 718, 625, 706 and Various Derivatives, ed. E. A. Loria, 1994, pp. 293-302, TMS (1997).

[67] Effect of hot deformation parameters on the grain size of wrought IN 718, J. M. Zhang et al., Proceedings of the 4th International Symposium on Superalloys 718, 625, 706 and Various Derivatives, ed. E. A. Loria, Pittsburgh, Pennsylvania, June, 1997, pp. , TMS (1997).

[68] Predicting grain size evolution of UDIMET alloy 718 during the "Cogging" process throught the use of Numerical Analysis, B. Antolovich, M. Evans, Proceedings of the 9th International Symposium on Superalloys 718, 625, 706 and Various Derivatives, ed. T. M. Pollack, et al., Champion, Pennsylvania, September, 2000, pp. 39-47, TMS (2000).

[69] Computer simulation of the forging of fine grain IN-718 alloy, R. Srinivasan, et al., Metallurgical and Materials Transactions A, Vol. 24 (9), pp. 2061-2069, Springer (1993).

[70] Microstructural simulation of nickel based Inconel 718 in production of turbine discs, A. J. Brand, et al., Materials Science and Technology, Vol. 12 (11), pp. 963-969, Maney (1996).

[71] Comparison of numerical results with the experimental results on flow stress and microstructural evolution of super alloy 718, M. Kawano, S. Isogawa, Simulation of Material Processing: Theory, Methods and Applications, ed. K. Mori, et al., pp. 269-274, Taylor \& Francis (2001).

[72] Microstructural modelling of metadynamic recrystallization in hot working of IN-718 superalloy, S. C. Medeiros et al., Materials Science and Engineering A, Vol. 293 (1-2), pp. 198-207, Elsevier (2000).

[73] Microstructural evolution of Inconel 718 during ingot breakdown: process modelling and validation, C. A. Dandre et al., Materials science and Technology, Vol. 16 (1), pp. 14-26, Maney (2000).

[74] Integral modeling of texture evolution in multiple pass hot rolling in aluminiun alloys, M. Goerdeler et al., Material Science Forum, vol. 396-402, pp. 379-386, Trans Tech (2002).

[75] Prediction of temperature evolution by FEM during multi-pass hot flat rolling of aluminum alloys, X. Duan, T. Sheppard, Modeling and Simulation in Materials Science and Engineering, Vol. 9 (6), pp. 525-538, Institute of Physics (2001). 
[76] An internal variable constitutive model for hot working of metals, S. B. Brown, et al., International Journal of Plasticity, Vol. 5 (2), pp. 95-130, Elsevier (1989).

[77] Multiphase rolling of aluminium alloys: finite element simulation and microstructural evolution, M. S. Mirza et al., Materials Science and Technology, Vol. 17 (7), pp. 874-879, Maney (2001).

[78] Microstructural simulation during hot rolling of Al-Mg Alloys, J. Hirch, et al., Proceedings of the 4th International Conference on Aluminium Alloys, Atlanta, Georgia, 1994, pp. 476-483.

[79] Coupling of atomistic and continuum simulations using a bridging scale decomposition, G. J. Wagner, W. K. Liu, Journal of Computational Physics, Vol. 190 (1), pp. 249-274, Elsevier (2003).

[80] A multi-sale approach for microstructure prediction in thermo-mechanical processing of metals, Q. Yu, S. K. Esche, Journal of Material Processing Technology, Vol. 169 (3), pp. 493-502, Elsevier (2005).

[81] Computer simulation of grain growth, H. J. Frost, C. V. Thompson, Current Opinion in Solid State \& Materials Science, Vol. 1 (3), pp. 361-368, Elsevier (1996).

[82] Computer simulation of grain growth - I. Kinetics, M. P. Anderson, et al., Acta Metallurgica, Vol. 32 (5), pp. 783-791, Elsevier (1983).

[83] Grain boundary $\gamma$ " precipitation and niobium segregation in Inconel 718, M. Gao, R. P. Wei, Scripta Metallurgica et Materialia, Vol. 32 (7), pp. 987-990, Elsevier (1995).

[84] Stereological estimation of microstructural parameters of nickel-based superalloy waspaloy using TEM methods, J. Wosik, et al., Materials characterization, Vol. 46 (2-3), pp. 119-123, Elsevier (2001).

[85] Quantitative microstructural characterization of Ni-base superalloys, H. J. Penkalla, et al., Materials Chemistry and Physics, Vol. 81 (2), pp. 417-423, Elsevier (2003).

[86] Elements of Materials Science, V. Vlack, Addison-Wesley (1967).

[87] Engineering Materials: An Introduction to Their Properties and Applications, M. F. Ashby, D. R. H. Jones, International Series on Material Science and Technology, Vol. 34, Oxford Pergamon (1986).

[88] Structure and Properties of Engineering Materials, R. M. Brick, et al., Metallurgy and Metallurgical Engineering Series, Mc Graw Hill (1977).

[89] Structure and Properties of Alloys: The application of phase diagrams to the interpretation and control of industrial alloy structures, R. M. Brick, et al., Materials Science and Engineering Series Mc Graw Hill (1965). 
[90] Phase transformations in metals and alloys, D. A. Porter and K. E. Easterling, CRC (1992).

[91] Phase Diagrams in Metallurgy, F. M. Rhines, Metallurgy and Metallurgical Engineering Series, Mc Graw Hill (1956).

[92] Introduction to Physical Metallurgy, S. H. Avner, McGraw Hill (1974).

[93] Fundamentals of Materials Science and Engineering: An Integrated Approach, W. D. Callister, Jr., Wiley (2005).

[94] Recrystallization and Related Annealing Phenomena, F. J. Humphreys and M. Hatherly, Elsevier (2004).

[95] Deformation and fracture mechanics of engineering materials, R. W. Hertzberg, Wiley (1996). 